\title{
Identification of best available thermal energy storage compounds for low-to-moderate temperature storage applications in buildings
}

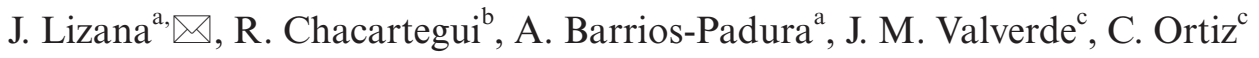 \\ a. Escuela Técnica Superior de Arquitectura, Universidad de Sevilla (Seville, Spain) \\ b. Escuela Técnica Superior de Ingeniería, Universidad de Sevilla, (Seville, Spain) \\ c. Facultad de Física, Universidad de Sevilla, (Seville, Spain) \\ \llizana@us.es
}

\begin{abstract}
Over the last 40 years different thermal energy storage materials have been investigated with the aim of enhancing energy efficiency in buildings, improving systems performance, and increasing the share of renewable energies. However, the main requirements for their efficient implementation are not fully met by most of them. This paper develops a comparative review of thermophysical properties of materials reported in the literature. The results show that the highest volumetric storage capacities for the best available sensible, latent and thermochemical storage materials are $250 \mathrm{MJ} / \mathrm{m}^{3}, 514 \mathrm{MJ} / \mathrm{m}^{3}$ and $2000 \mathrm{MJ} / \mathrm{m}^{3}$, respectively, corresponding to water, barium hydroxide octahydrate, and magnesium chloride hexahydrate. A group of salt hydrates and inorganic eutectics have been identified as the most promising for the development of competitive thermal storage materials for cooling, heating and comfort applications in the short-term. In the long-term, thermochemical storage materials seem promising. However, additional research efforts are required.
\end{abstract}

KEYWORDS: Thermal energy; Characterization; Thermal energy storage materials; Phase change materials; Thermophysical properties.

Citation/Citar como: Lizana, J.; Chacartegui, R.; Barrios-Padura, A.; Valverde, J.M.; Ortiz, C (2018) Identification of best available thermal energy storage compounds for low-to-moderate temperature storage applications in buildings. Mater. Construcc. 68 [331], e160 https://doi.org/10.3989/mc.2018.10517

RESUMEN: Identificación de los mejores compuestos disponibles de almacenamiento de energía térmica para aplicaciones de baja a moderada temperatura en edificación. En los últimos 40 años se han investigado diferentes materiales de almacenamiento térmico con el objetivo de mejorar la eficiencia energética en los edificios, mejorar el rendimiento de sistemas y aumentar el uso de renovables. Sin embargo, la mayoría no cumple los principales requisitos para su eficiente implementación. Este artículo desarrolla una revisión de las propiedades termofísicas de los materiales existentes en la literatura. Los resultados muestran que las mayores capacidades de almacenamiento volumétrico para los mejores materiales de almacenamiento sensible, latente y termoquímico son 250 $\mathrm{MJ} / \mathrm{m}^{3}, 514 \mathrm{MJ} / \mathrm{m}^{3}$ y $2000 \mathrm{MJ} / \mathrm{m}^{3}$, respectivamente, correspondientes a agua, hidróxido de bario octahidratado y cloruro de magnesio hexahidratado. Un conjunto de sales hidratadas y eutécticos han sido identificados como los más prometedores para el desarrollo de materiales competitivos para aplicaciones de enfriamiento, calefacción y confort a corto plazo. A largo plazo, el almacenamiento termoquímico parece prometedor. Sin embargo, investigación adicional es requerida.

Palabras clave: Energía térmica; Caracterización; Materiales de almacenamiento de energía térmica; Materiales de cambio de fase; Propiedades termofísicas,

ORCID ID: J. Lizana (http://orcid.org/0000-0002-1802-5017); R. Chacartegui (http://orcid.org/0000-0001-7285-8661); A. Barrios-Padura (http://orcid.org/0000-0003-1054-010X); J. M. Valverde (http://orcid.org/0000-0002-2345-8888); C. Ortiz (http://orcid.org/0000-0002-7795-676X)

Award-winning paper at III International Congress and V National on Sustainable Construction and Eco-Efficient Solutions (CICSE) March 2017

Copyright: (C) 2018 CSIC. This is an open-access article distributed under the terms of the Creative Commons Attribution 4.0 International (CC BY 4.0) License. 


\section{Nomenclature}

\begin{tabular}{|c|c|}
\hline$c_{p}$ & specific heat $(\mathrm{KJ} / \mathrm{kg} \mathrm{K})$ \\
\hline CSMP & composite salt in porous matrix \\
\hline DH & district heating \\
\hline$h$ & latent heat of fusion per unit mass $(\mathrm{kJ} / \mathrm{kg})$ \\
\hline HVAC\&R & $\begin{array}{l}\text { heating, ventilation, air conditioning and } \\
\text { refrigeration }\end{array}$ \\
\hline LHSM & latent heat storage materials \\
\hline$m$ & mass of heat storage medium $(\mathrm{kg})$ \\
\hline PCM & phase change material \\
\hline PEG & polyethylene glycol \\
\hline$Q$ & quantity of heat stored (MJ) \\
\hline SHSM & sensible heat storage material \\
\hline $\mathrm{T}$ & temperature $\left({ }^{\circ} \mathrm{C}\right)$ \\
\hline TABS & thermally Activated Building system \\
\hline TCA & thermo-chemical accumulator \\
\hline TCES & thermochemical energy storage \\
\hline TES & thermal energy storage \\
\hline TSM & thermochemical energy storage material \\
\hline UTES & underground thermal energy storage \\
\hline
\end{tabular}

Greek letters

$\begin{array}{ll}\alpha & \text { thermal diffusivity }\left(\mathrm{mm}^{2} / \mathrm{s}\right) \\ \lambda & \text { thermal conductivity }(\mathrm{W} / \mathrm{m} \mathrm{K}) \\ \rho & \text { density }\left(\mathrm{kg} / \mathrm{m}^{3}\right)\end{array}$

Subscript

$1 \quad$ liquid

\section{INTRODUCTION}

Over the last 40 years, a number of thermal energy storage and management technologies have been explored for a low-carbon energy transition in domestic and industrial sectors given their great potential to reduce energy demand, increase the use of renewable energy and improve the operating conditions of heating and cooling systems. Probably, thousands of pure materials and mixtures have been investigated for their use in thermal energy storage (TES) applications $(1,2)$. From the early publications of Abhat (3, 4), Lane (5), Telkes $(6,7)$, Schröder and Gawron (8) and Naumman and Emons (9) in the 1980s, until most recent works of Sharma et al. $(10,11)$, Cabeza et al. (12), Tyagi et al. (13, 14), Zhou et al. (15), N'Tsoukpoe et al. (16), Tatsidjodoung et al, Yu et al. (17), De Gracia and Cabeza (18), Kenisarin and Mahkamov $(19,20)$ and Alva et al. $(21,22)$, different types of thermal storage compounds have been considered, tested and listed as promising for low-to-moderate temperature applications. This includes liquid and solid materials for sensible storage, organic and inorganic materials, and their eutectic mixtures, for latent heat storage and thermochemical storage forms. For an efficient implementation of these systems, they should possess a high volumetric TES density with high thermal stability and reliability under cycling, as well as being widely present readily available at low cost (1). However, these requirements are not fully met by most existing materials.

The aim of this paper is to identify the best available TES compounds developed for low-to-moderate temperature storage applications. The work considers all existing and tested to our knowledge sensible, latent and thermochemical TES materials in the temperature range between 0 and $100{ }^{\circ} \mathrm{C}$. The paper is structured as follows. First, heat storage methods and their main building applications are introduced. Afterwards, definition of characterization parameters and requirements of each storage method are provided. Most of the TES materials tested and reported in the literature are categorized, with special emphasis on their thermophysical properties. Finally, all the collected materials are compared, according to volumetric TES capacity and temperature applications, and the compounds with highest potential are identified.

\section{CLASSIFICATION OF THERMAL ENERGY STORAGE MATERIALS AND THEIR BUILDING APPLICATIONS}

Thermal energy storage methods can be broadly classified as sensible, latent (phase change) and thermochemical. Figure 1 shows the main available material groups for each storage method in building applications.

Sensible heat storage is just based on increasing or decreasing the temperature of a given material with high heat capacity. Sensible storage compounds are usually classified into liquids (such as water or oil) and solids (such as rocks, concrete, bricks, sand or gravel). Latent heat storage relies on the heat absorbed or released when a material undergoes a phase change from a physical state to another $(15,23)$. Phase change materials (PCMs) are classified into organics (paraffins, fatty acids, esters, sugar alcohols, polyethylene glycols and other), inorganics (salt hydrates and metals) and eutectics mixtures (mixtures of inorganics and/or organics) $(4,15)$. Thermochemical energy storage (TCES) is based on the use of a source of energy to induce a reversible chemical reaction and/ or sorption process (17). Thermochemical storage materials (TSM) can be classified into solid adsorption materials, liquid absorption materials, chemically reactive solid materials and composite materials (CSMP). 


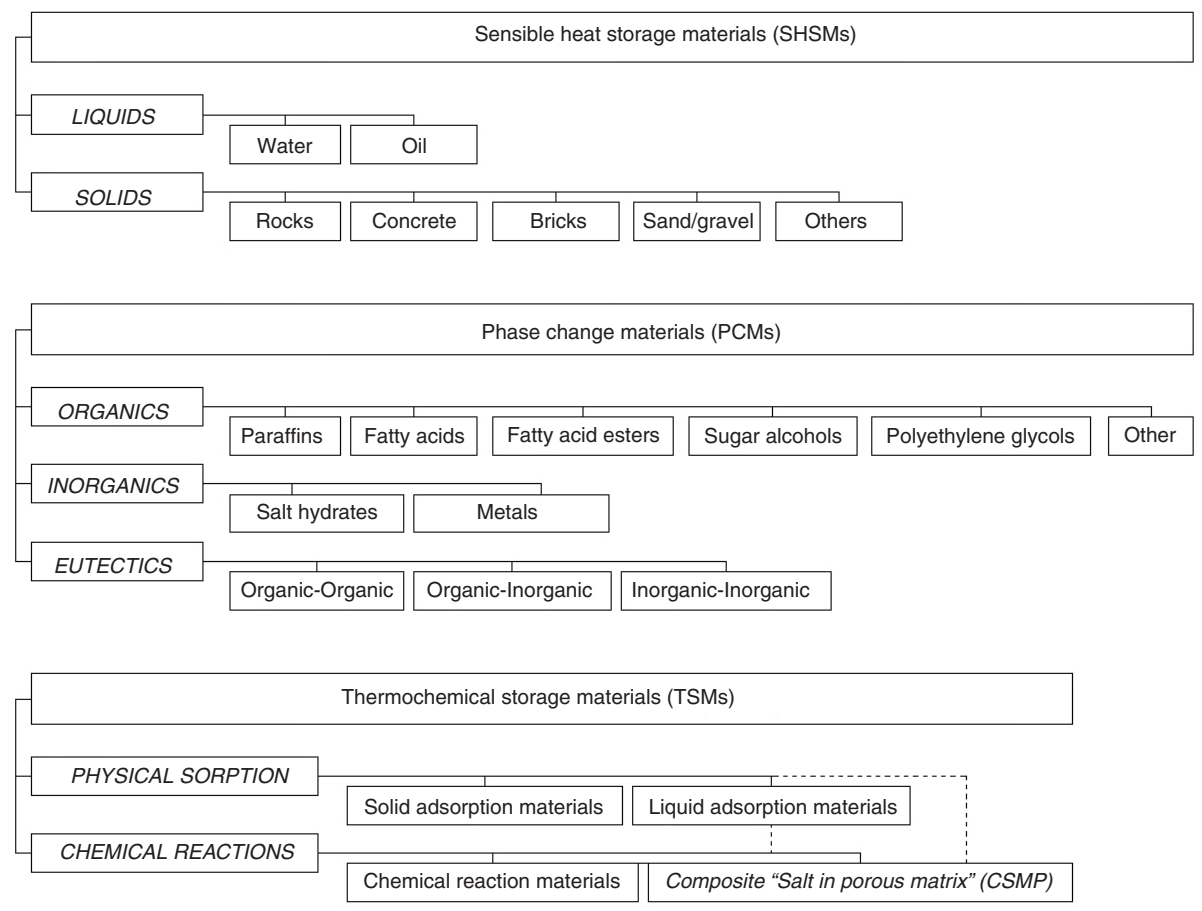

FIGURE 1. Classification of thermal energy storage materials.

TES materials can be integrated in buildings to improve their energy performance by means of different passive or active applications.

- Passive solutions are characterized by a heat exchange without mechanical action via natural convection between the indoor environment and storage material (24).

- Active applications are based on a heat exchange assisted by a mechanical component (fan, blower or pump) (25).

Figure 2 illustrates the relationships existing between available TES materials and their possible applications. Main building applications can be classified into: TES in building materials or elements, thermally activated building systems (TABSs), TES components, small-scale TES units and large-scale TES systems (26).

TES in building materials or elements consists of passive applications based on high thermal inertia. They allow improving the thermal performance of buildings by the attenuation of thermal oscillations (24). TABSs consist of active applications which use the building structure as a TES system. A thermal energy source is connected to a construction element which exchanges heat with the indoor space, such as an underfloor heating system (27). TES components consist of latent heat storage modules usually applied to ventilation systems in free cooling applications (28), and PV panels for increasing the electrical yield thanks to a reduction of temperature rise of their surface (29-31). Small-scale TES units refers to compact storage tanks, which allow increasing the use of renewable energy and improving the energy performance of heating, ventilation, air conditioning and refrigeration (HVAC\&R) systems $(32,33)$. Large-scale TES systems refer to above-ground large-scale tanks or underground thermal energy storage (UTES) systems (34). Their main applications consist of solar storage in district heating (DH) networks or large-scale buildings. Following this classification, most advanced TES solutions assessed and tested in literature was further assessed by Lizana et al. (35).

Each application group has different temperature ranges according to final implementation and thermal energy use. Also, the performance of these TES applications depends on the thermophysical properties of the TES materials chosen.

\section{CHARACTERIZATION PARAMETERS OF TES MATERIALS}

\subsection{Sensible and latent heat storage materials}

Sensible heat storage is yet the most widely used technique for TES in buildings while applications based on latent heat storage are gradually growing as they allow storing high thermal energy amounts within a narrower temperature range. Characterization parameters of sensible and latent 


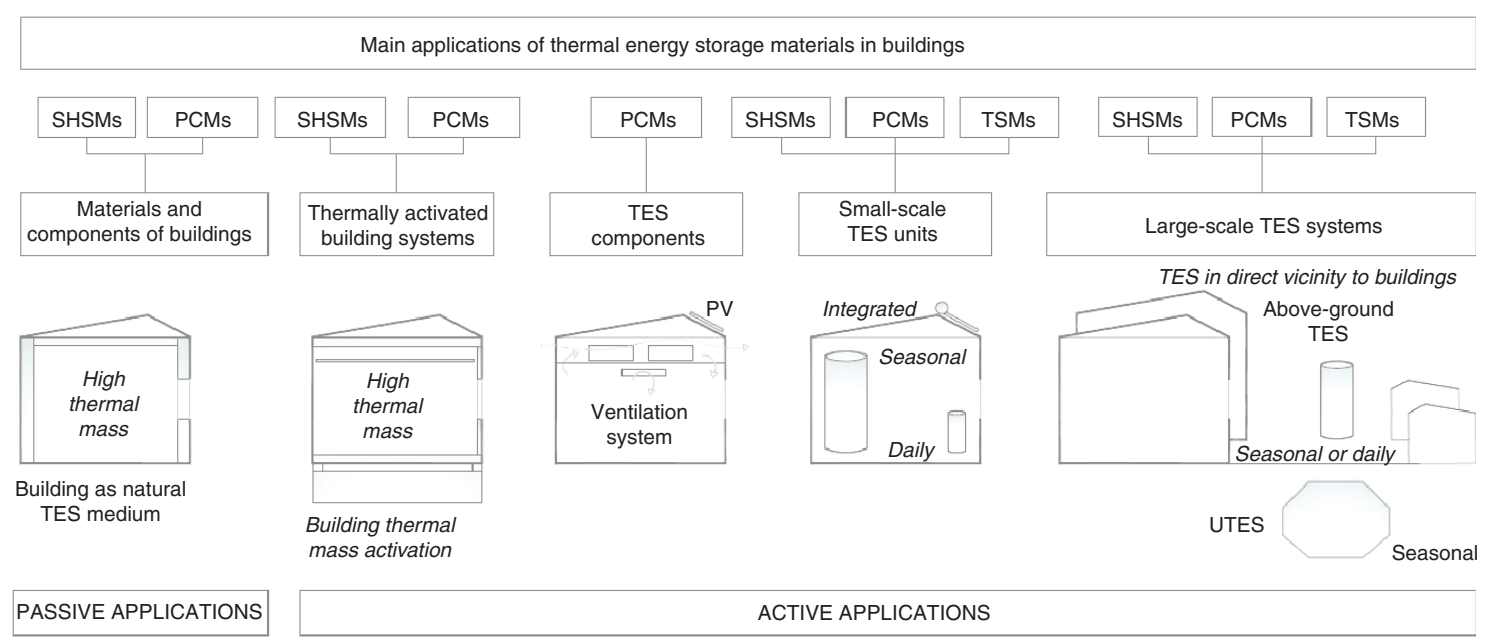

FIGURE 2. Applications of thermal energy storage materials in buildings.

heat storage materials (SHSMs and LHSMs), and their influences in storage solutions are showed in Table $1(2,23,36)$.

The amounts of energy stored in a given mass of material $(m)$ by sensible heat storage $\left(Q_{\text {sensible }}\right)$ and by latent heat storage $\left(Q_{\text {latent }}\right)$ are given by Eq. [1] and Eq. [2] (23), respectively.

$Q_{\text {sensible }}=m c_{p} \Delta T(M J)$

$Q_{\text {latent }}=m\left[\left(c_{p_{s}} \Delta T\right)_{\text {sensible }}+(h)_{\text {latent }}+\left(c_{p l} \Delta T\right)_{\text {sensible }}\right](M J)$

The most important physical properties to take into consideration are: i) high specific heat capacity and density, good thermal conductivity and low cost for sensible heat storage; ii) suitable phase change temperature for the corresponding application, high volumetric latent heat capacity, good thermal conductivity and low cost for latent heat storage. As a reference value, sensible heat storage capacity of water, for a temperature difference $(\Delta T)$ of $60{ }^{\circ} \mathrm{C}\left(25-85^{\circ} \mathrm{C}\right)$, is $250 \mathrm{MJ} / \mathrm{m}^{3}$. This value should be considered as maximum reference value, which could be reduced according to final building application.

Other requirements and constraints for selecting SHSMs and LHSMs are specified in Table $2(2,38)$. They are based on safety conditions, environmental impact and compatibility with other materials.

TABLE 1. Characterization parameters of SHSMs and LHSMs

\begin{tabular}{|c|c|c|}
\hline Property & Measure & Influences \\
\hline Density $(\rho)$ & $\mathrm{kg} / \mathrm{m}^{3}$ & \\
\hline Specific heat $\left(c_{p}\right)$ & $\mathrm{kJ} / \mathrm{kg} \mathrm{K}$ & Thermal storage capacity \\
\hline Latent heat of phase change $(h)$ & $\mathrm{kJ} / \mathrm{kg}$ & \\
\hline Phase change temperature & ${ }^{\circ} \mathrm{C}$ & Thermal application \\
\hline Thermal conductivity $(\lambda)$ & $\mathrm{W} / \mathrm{m} \mathrm{K}$ & \\
\hline Thermal diffusivity $(\alpha)$ & $\mathrm{mm}^{2} / \mathrm{s}$ & Charging/ discharging time Stratification ability \\
\hline Thermal effusivity & $\mathrm{W} \sqrt{s} / m^{2 o} C$ & Ability to exchange thermal energy with its surroundings (36) \\
\hline Thermal expansion coefficient & $\%$ & Change of volume (Requirements for container) \\
\hline $\begin{array}{l}\text { Thermal reliability } \\
\text { (Efficiency after thermal cycles) }\end{array}$ & $\%$ & Performance over several thermal cycles \\
\hline $\begin{array}{l}\text { Chemical stability } \\
\text { (after thermal cycles) }\end{array}$ & Changes in spectrum & No chemical decomposition of material after thermal cycles \\
\hline $\begin{array}{l}\text { Thermal stability }{ }^{\mathrm{b}} \\
\text { (Degradation at high temperature) }\end{array}$ & Weight loss $\%$ & No degradation of material with the increase of temperature \\
\hline
\end{tabular}

${ }^{\mathrm{a}} 5000$ cycles are required for approximately $13-14$ years (37).

${ }^{\mathrm{b}}$ Commonly not important for building applications due to low-temperature of applications. 
TABLE 2. Requirements or constraints for selecting of SHSMs and LHSMs

\begin{tabular}{|c|c|c|}
\hline Requirements or constraints & Measure & Reasons \\
\hline Small volume change & $\%$ & Less mechanical requirements of container \\
\hline Non-toxic, non-flammable, non-explosive and non-reactive & - & Safety \\
\hline Recyclability & $\%$ & \\
\hline Non-polluting, low $\mathrm{CO}_{2}$-eq footprint & $\mathrm{CO}_{2}-\mathrm{eq} / \mathrm{kg}$ & Environmental impact \\
\hline Non-corrosiveness & - & Compatibility with other materials \\
\hline Availability and low price & $€ / \mathrm{m}^{3}$ or $€ / \mathrm{kg}$ & Competitiveness and effective cost \\
\hline $\begin{array}{l}\text { Congruent melting } \\
\text { Not subcooling/supercooling }\end{array}$ & $\Delta \mathrm{T}^{\mathrm{a}}\left({ }^{\circ} \mathrm{C}\right)$ & $\begin{array}{l}\text { To assure that melting and solidification can } \\
\text { proceed in a narrow temperature range. }\end{array}$ \\
\hline Not phase segregation or separation ${ }^{\mathrm{b}}$ & - & Assure a long lifetime \\
\hline
\end{tabular}

${ }^{\mathrm{a}}$ Subcooling refers to a liquid existing at a temperature below its normal melting temperature. If such temperature is not reached, PCM will not solidify at all and stored heat will not be released (2).

${ }^{b}$ Phase segregation or separation refers to the conversion of a single-phase system into a multi-phase system (separation of components of a solution).

Main drawbacks of SHSMs are related to their low energy density (owing to space limitation for building applications) and system's self-discharge, which can be substantial due to heat losses (particularly for long-period storage) (38). Main drawbacks of LHSMs are related to their high cost, low thermal conductivity, relative large volume change, flammability, supercooling, corrosiveness, and thermal reliability and stability after undergoing a great number of thermal cycles (15).

\subsection{Thermochemical storage materials}

TCES can be classified into chemical reactions and/or sorption processes (17). A chemical reaction is characterized by a change in chemical bounds of the compounds involved during the reaction (dissociation and recombination). Energy can be stored through the endothermic reaction and released by the exothermic reverse reaction. Sorption storage can be defined as a phenomenon of fixation or capture of gas or vapour by a sorbent substance in the condensed state (solid or liquid) by means of less intense interactions. Also, sorption processes can involve thermophysical and thermochemical aspects (16). For the characterization of solid sorbents for thermal applications, in addition to energy density or process efficiency, other parameters should be considered: the possible temperature lift (defined as the difference between the air entering and exiting the solid bed), the drop in humidity ratio and the breakthrough curves (dynamic response of the system) (39). All these parameters can be known from the adsorption equilibrium of each adsorbent material.

Main advantages of TCES are high stored energy density, negligible heat losses, and long-term storage capacity. Characterization parameters of TSMs and their influences are showed in Table 3.
Thermal energy stored during a specific time period through thermochemical processes is given by Eq. [3] (23).

$$
\begin{aligned}
& C_{1} C_{2}+\text { Heat input } \Rightarrow C_{1}+C_{2} \rightrightarrows \text { storage } \rightrightarrows \\
& C_{1}+C_{2} \Rightarrow C_{1} C_{2}+\text { Heat output }
\end{aligned}
$$

Other requirements for selecting TSMs are defined in Table 4. They are based on safety conditions, environmental impact, lifetime and compatibility with other materials.

Main drawbacks of TSMs are related to their high cost, inappropriate operation temperatures, non-effective discharge power for building applications due to slow reaction kinetics and low output temperature close to the equilibrium point, and low/moderate efficiency in storage process.

\section{CHARACTERIZATION OF AVAILABLE THERMAL ENERGY STORAGE COMPOUNDS FOR BUILDING APPLICATION}

\subsection{Sensible heat storage materials}

SHSMs can be classified into liquid and solid storage materials (23). Usual liquid storage materials for low-to-moderate temperature applications are water, oils and alcohol derivatives. Some common solid storage materials are rocks, bricks, concrete, dry and wet earth/soil, wood, plasterboard, and corkboard. Some of the most commonly used in buildings are listed in Tables 5 and 6.

\subsubsection{Liquids}

Liquid materials are widely used for cooling and heating purposes. Water is the best available liquid material for temperature applications lower than $100{ }^{\circ} \mathrm{C}$ due to its high specific heat capacity, 
TABLE 3. Characterization parameters of TSMs

\begin{tabular}{|c|c|c|}
\hline Property & Measure & Influences \\
\hline Density $(\rho)$ & $\mathrm{kg} / \mathrm{m}^{3}$ & \multirow{5}{*}{ Thermal storage capacity } \\
\hline Heat input & $\mathrm{Wh} / \mathrm{kg}-\mathrm{MJ} / \mathrm{m}^{3}$ & \\
\hline Heat output (storage density) & $\mathrm{Wh} / \mathrm{kg}-\mathrm{MJ} / \mathrm{m}^{3}$ & \\
\hline Storage efficiency $\left(\mathrm{Q}_{\text {released }} / \mathrm{Q}_{\text {stored }}\right)$ & $\%$ & \\
\hline $\begin{array}{l}\text { Degree of sorbate loading in the adsorption/absorption } \\
\text { equilibrium }\end{array}$ & $\begin{array}{l}\% \\
\text { (for physical sorption) }\end{array}$ & \\
\hline Charging/desorption temperature & ${ }^{\circ} \mathrm{C}$ & \multirow{3}{*}{ Thermal application } \\
\hline Discharging/sorption temperature & ${ }^{\circ} \mathrm{C}$ & \\
\hline Temperature lift $\left(T_{\text {air, in }}-T_{\text {air out }}\right)$ & ${ }^{\circ} \mathrm{C}$ & \\
\hline Thermal conductivity $(\lambda)$ & $\mathrm{W} / \mathrm{m} \mathrm{K}$ & \multirow{3}{*}{ Charging/ discharging time } \\
\hline Kinetic of reaction or reaction rate & $\mathrm{m} / \mathrm{s}$ & \\
\hline $\begin{array}{l}\text { Evolution of output temperature close to the } \\
\text { equilibrium point }\end{array}$ & ${ }^{\circ} \mathrm{C}$ & \\
\hline Operating pressure range & $\mathrm{Pa}$ & System design requirements \\
\hline Thermal reliability (Efficiency after thermal cycles) & $\%$ & Performance over several cycles \\
\hline Chemical stability (After thermal cycles) & $\begin{array}{l}\text { Changes in spectrum after } \\
\text { thermal cycles }\end{array}$ & $\begin{array}{l}\text { No chemical decomposition of material } \\
\text { after thermal cycles }\end{array}$ \\
\hline Thermal stability & Weight loss $\%$ & $\begin{array}{l}\text { No degradation of material with the } \\
\text { increase of temperature }\end{array}$ \\
\hline
\end{tabular}

TABLE 4. Requirements or constraints for selecting of TSMs

\begin{tabular}{lll}
\hline Requirements or constraints & \multicolumn{1}{c}{ Measure } & \multicolumn{1}{c}{ Reasons } \\
\hline Non-toxic, non-flammable, non-explosive and non-reactive & - & Safety \\
Non-polluting, low $\mathrm{CO}_{2}$-eq footprint & $\mathrm{CO}_{2}$-eq/kg & Environmental impact \\
Non-corrosiveness & - & Compatibility with other materials \\
Availability and low price & $€ / \mathrm{m}^{3}$ & Competitiveness and effective cost \\
Moderate operating pressure range & $\mathrm{Pa}$ & $\begin{array}{l}\text { No excessive pressure conditions and especially } \\
\text { no high vacuum. Less system requirements }\end{array}$ \\
\hline
\end{tabular}

Table 5. SHSMs. Liquids. Properties

\begin{tabular}{|c|c|c|c|c|c|}
\hline Material & Density $\left(\mathrm{kg} / \mathrm{m}^{3}\right)$ & $\begin{array}{c}\text { Thermal conductivity } \\
(\mathrm{W} / \mathrm{m} \mathrm{K})\end{array}$ & $\begin{array}{c}\text { Specific heat } \\
(\mathbf{k J} / \mathbf{k g ~ K})\end{array}$ & $\begin{array}{c}\text { Average volumetric specific } \\
\text { heat capacity }\left(\mathrm{kJ} / \mathrm{m}^{3} \mathrm{~K}\right)\end{array}$ & Reference \\
\hline Mineral oil & 770 & 0.12 & 1.96 & 1509 & $(21)$ \\
\hline Engine Oil & 888 & 0.14 & 1.88 & 1669 & (38) \\
\hline Water $\left(80^{\circ} \mathrm{C}\right)$ & 970 & 0.67 & 4.19 & 4064 & $(40)$ \\
\hline Water $\left(40^{\circ} \mathrm{C}\right)$ & 990 & 0.63 & 4.19 & 4148 & $(40)$ \\
\hline Water $\left(10^{\circ} \mathrm{C}\right)$ & 1000 & 0.6 & 4.19 & 4190 & (40) \\
\hline
\end{tabular}

availability and low cost (38). It is the most widely used medium for heating and cooling applications as it can be circulated easily to transport heat if required (21). For a temperature gradient of $60{ }^{\circ} \mathrm{C}$ $\left(25-85^{\circ} \mathrm{C}\right)$, water stores $250 \mathrm{MJ} / \mathrm{m}^{3}$. This value is taken into account as a reference for comparison with other TES materials. Main drawbacks are related to the risk of leakages and high investment cost of storage infrastructures mainly for largescale applications.

\subsubsection{Solids}

Solid materials are widely used for heating applications at low and high temperature. Rocks and concrete can operate in a temperature range from 
TABle 6. SHSMs. Solids. Properties

\begin{tabular}{lccccc}
\hline Material & $\begin{array}{c}\text { Density } \\
\left(\mathbf{k g} / \mathbf{m}^{\mathbf{3}}\right)\end{array}$ & $\begin{array}{c}\text { Thermal conductivity } \\
(\mathbf{W} / \mathbf{m} \mathbf{~ K})\end{array}$ & $\begin{array}{c}\text { Specific heat } \\
(\mathbf{k J} / \mathbf{k g ~ K})\end{array}$ & $\begin{array}{c}\text { Average volumetric specific } \\
\left.\text { heat capacity (kJ/m } \mathbf{K}^{3}\right)\end{array}$ & Reference \\
\hline Wood (low density) & 450 & 0.12 & 1.6 & 720 & $(40)$ \\
Plywood boards (low density) & 500 & 0.13 & 1.6 & 800 & $(40)$ \\
Gypsum (plasterboard) & 900 & 0.25 & 1 & 900 & $(40)$ \\
Gypsum (coating) & 1000 & 0.4 & 1 & 1000 & $(40)$ \\
Oriented strand board & 600 & 0.14 & 1.7 & 1120 & $(40)$ \\
Wood (high density) & 700 & 0.18 & 1.6 & 1530 & $(40)$ \\
Oriented strand board & 900 & 0.18 & 1.7 & 1600 & $(40)$ \\
Ceramic tile & 2000 & 1 & 0.8 & 1600 & $(40)$ \\
Lime mortar & 1600 & 0.8 & 1 & 1600 & $(40)$ \\
Plywood boards (high density) & 1000 & 0.24 & 1.6 & 1656 & $(40)$ \\
Ceramic brick & 1800 & 0.73 & 0.92 & 1800 & $(41)$ \\
Cement bonded particleboard & 1200 & 0.23 & 1.5 & 1800 & $(40)$ \\
Cement mortar & 1800 & 1 & 1 & 2000 & $(40)$ \\
Concrete & 2000 & 1.35 & 1 & 2072 & $(40)$ \\
Sand and gravel & $1700-2200$ & 2 & $0.910-1.180$ & 2100 & $(40)$ \\
Limestone & $1600-2600$ & $0.85-2.3$ & 1 & 2150 & $(40)$ \\
Rock & $2800-1500$ & $3.5-0.85$ & 1 & 2400 & $(40)$ \\
Concrete (high density) & 2400 & 2 & 1 & 2400 & $(40)$ \\
Reinforced concrete (2\%) & 2400 & 2.5 & $1.670-2.500$ & 3252 & $(40)$ \\
Clay or silt & $1200-1800$ & 1,5 & 1.7 & 3910 & $(40)$ \\
Asphalt sheet & 2300 & 1.2 & & $(42)$ \\
\hline
\end{tabular}

40 to $75^{\circ} \mathrm{C}$ without any leakage problem $(23,38)$. They have a reduced energy storage density for heating as compared to water $\left(95-105 \mathrm{MJ} / \mathrm{m}^{3}\right)$, however stratification can be maintained over considerable time periods due to the high thermal diffusivity $\left(0.7-1 \mathrm{~mm}^{2} / \mathrm{s}\right)$, which helps heat release and charge (38). Solids are also characterized by some limitations such as, associated costs involved in operation and maintenance of the storage units, and risks of self-discharge in long-term storage.

Other solids materials widely used in buildings to improve TES capacity are brick, sand and gravel, cement, gypsum and bricks. They have been mainly applied to improve the thermal inertia of building structure, for attenuating indoor temperature oscillation. The properties of common sensible building materials are listed in Table 6.

\subsection{Latent heat storage materials}

Solid-liquid PCMs are the most common processes in building applications. This section reviews all different latent heat compounds, eutectics and mixtures that have been studied by different researchers for their potential use as PCMs with a special focus on their thermophysical properties.

\subsubsection{Organics}

Organic materials have been successfully tested and implemented in many building applications (21). They present congruent melting without phase separation. Organic materials used as PCM are commonly classified into paraffins and non-paraffins. Paraffins or paraffin waxes refer to linear chain hydrocarbons composed of n-alkanes. Non-paraffins usually refer to a large category of organic materials for latent heat storage composed mainly by fatty acids, esters, sugar alcohols, glycols and other (10).

4.2.1.1. Paraffins Paraffins or straight-chain alkanes are characterized by the chemical formula $\mathrm{C}_{\mathrm{n}} \mathrm{H}_{2 \mathrm{n}+2}$ or $\mathrm{CH}_{3}-\left(\mathrm{CH}_{2}\right)_{\mathrm{n}}-\mathrm{CH}_{3}(10,11)$. Pure paraffin waxes are very expensive, thus only technical grade paraffins are commonly used as PCMs due to cost considerations $(10,21)$. They are widely utilized as PCMs because of their favorable properties: moderate thermal storage density (approximately $200 \mathrm{~kJ} / \mathrm{kg}$ and $150 \mathrm{MJ} / \mathrm{m}^{3}$ ), congruent melting and solidification with negligible subcooling (good nucleating properties), good chemical stability without phase segregation, little volume change, non-corrosive with metal containers, non-toxic and moderate 
cost $(4,10,21,38,43,44)$. They usually show stable properties and good thermal reliability even after 1000-2000 thermal cycles (21). However, they have low thermal conductivity $(\approx 0.2 \mathrm{~W} / \mathrm{mK})$, which reduces their heat exchange rate during the melting and solidification processes $(1,38)$. In addition, they show moderate flammability and can interact with some plastic containers, particularly with polyolefins, leading to infiltrations and softening $(1,10)$.

Table 7 shows the straight-chain alkanes tested in literature and their performance according to the results obtained by different authors.

TABle 7. Organic PCMs: straight-chain alkanes. Properties (solid/liquid)

\begin{tabular}{|c|c|c|c|c|c|c|}
\hline Material & $\begin{array}{l}\text { Melting } \\
\text { temp. }\left({ }^{\circ} \mathbf{C}\right)\end{array}$ & $\begin{array}{l}\text { Density } \\
\left(\mathrm{kg} / \mathrm{m}^{3}\right)\end{array}$ & $\begin{array}{l}\text { Thermal conductivity } \\
\text { (W/m K) }\end{array}$ & $\begin{array}{l}\text { Specific heat } \\
(\mathrm{kJ} / \mathrm{kg} \mathrm{K})\end{array}$ & $\begin{array}{l}\text { Latent heat } \\
(\mathrm{kJ} / \mathrm{kg})\end{array}$ & Reference \\
\hline $\begin{array}{l}\text { n-Tridecane }\left(\mathrm{C}_{13} \mathrm{H}_{28}\right) \\
\mathrm{CH}_{3}\left(\mathrm{CH}_{2}\right)_{11} \mathrm{CH}_{3}\end{array}$ & -5 & & & & 160 & (21) \\
\hline \multirow{6}{*}{$\begin{array}{l}\text { n-Tetradecane }\left(\mathrm{C}_{14} \mathrm{H}_{30}\right) \\
\mathrm{CH}_{3}-\left(\mathrm{CH}_{2}\right)_{12}-\mathrm{CH}_{3}\end{array}$} & $4.49-11.72$ & & & & 230.38 & $(45)$ \\
\hline & 4.5 & & & & 165 & $(4,46)$ \\
\hline & 5.5 & & & & 228 & $(10)$ \\
\hline & 5.9 & $/ 762$ & & & & (47) \\
\hline & 6 & $/ 760$ & $0.21 /$ & & 230 & (48) \\
\hline & 6 & 810 & & & 229 & (49) \\
\hline \multirow{5}{*}{$\begin{array}{l}\text { n-Pentadecane }\left(\mathrm{C}_{15} \mathrm{H}_{32}\right) \\
\mathrm{CH}_{3}-\left(\mathrm{CH}_{2}\right)_{13}-\mathrm{CH}_{3}\end{array}$} & $8.72-15.11$ & & & & 176.15 & (45) \\
\hline & 9.6 & $776.1 / 727.2$ & $0.182 / 0.15$ & $3.08 / 3.53$ & 168.0 & $(50)$ \\
\hline & 9.9 & $/ 768.3$ & & & & (47) \\
\hline & 10 & $/ 770$ & & & 212 & (48) \\
\hline & 10 & & & & 205 & (10) \\
\hline \multirow{8}{*}{$\begin{array}{l}n \text {-Hexadecane }\left(\mathrm{C}_{16} \mathrm{H}_{34}\right) \\
\mathrm{CH}_{3}-\left(\mathrm{CH}_{2}\right)_{14}-\mathrm{CH}_{3}\end{array}$} & 16.7 & & & & 237.1 & (10) \\
\hline & $16.76-23.63$ & & & & 225.15 & (45) \\
\hline & 18 & 780 & $0.17-0.26$ & $1.65 / 2.1$ & 236 & $(51)$ \\
\hline & 18 & 1776 & $0.21 /$ & & 210.238 & (48) \\
\hline & 18 & 830 & & & 229 & (49) \\
\hline & $18.0-20.0$ & $/ 773$ & & & $216-236$ & $(1,52)$ \\
\hline & 18.2 & $/ 773.4$ & & & & (47) \\
\hline & 20 & & & & 185 & $(22,43)$ \\
\hline \multirow{6}{*}{$\begin{array}{l}n \text {-Heptadecane }\left(\mathrm{C}_{17} \mathrm{H}_{36}\right) \\
\mathrm{CH}_{3}-\left(\mathrm{CH}_{2}\right)_{15}-\mathrm{CH}_{3}\end{array}$} & 19 & 1776 & & & 240 & (48) \\
\hline & $21-27.36$ & & & & 172.21 & (45) \\
\hline & 21.7 & & & & 213 & (10) \\
\hline & 21.98 & 777.9/ & & & & (47) \\
\hline & 22 & 780 & $0.17-0.26$ & & 214 & $(51)$ \\
\hline & 22 & $/ 778$ & & & 214 & $(1,52)$ \\
\hline \multirow{9}{*}{$\begin{array}{l}n \text {-Octadecane }\left(\mathrm{C}_{18} \mathrm{H}_{38}\right) \\
\mathrm{CH}_{3}-\left(\mathrm{CH}_{2}\right)_{16}-\mathrm{CH}_{3}\end{array}$} & 27.74 & & 0.153 & & 209.10 & $(22,53)$ \\
\hline & 28.0 & & & & 244 & (10) \\
\hline & 28 & 780 & $0.17-0.26$ & $1.75 / 2.1$ & 244 & (51) \\
\hline & 28 & $814 / 774$ & $0.15 /$ & 12.16 & 244 & $(4,46,54)$ \\
\hline & 28 & $814 / 774$ & $0.358 / 0.148$ & & 200.245 & (48) \\
\hline & 28 & 850 & & & 243 & (49) \\
\hline & $28.0-28.4$ & /776 & & & $200-244$ & $(1,52)$ \\
\hline & 28.2 & $781.8 /$ & & & & $(47)$ \\
\hline & 28.4 & & & & 234 & $(22,43)$ \\
\hline \multirow{4}{*}{$\begin{array}{l}n \text {-Nonadecane }\left(\mathrm{C}_{19} \mathrm{H}_{40}\right) \\
\left(\mathrm{CH}_{3}\left(\mathrm{CH}_{2}\right)_{17} \mathrm{CH}_{3}\right)\end{array}$} & 31.9 & $785.4 /$ & & & & (47) \\
\hline & 32.0 & & & & 222 & (10) \\
\hline & 32.0 & $/ 785$ & & & 222 & $(1,52)$ \\
\hline & 32 & & & & 194.3 & $(22,43)$ \\
\hline
\end{tabular}


TABLE 7. Continued

\begin{tabular}{|c|c|c|c|c|c|c|}
\hline Material & $\begin{array}{l}\text { Melting } \\
\text { temp. }\left({ }^{\circ} \mathbf{C}\right)\end{array}$ & $\begin{array}{l}\text { Density } \\
\left(\mathrm{kg} / \mathrm{m}^{3}\right)\end{array}$ & $\begin{array}{c}\text { Thermal conductivity } \\
(\mathrm{W} / \mathrm{m} \mathrm{K})\end{array}$ & $\begin{array}{c}\text { Specific heat } \\
(\mathrm{kJ} / \mathrm{kg} \mathrm{K})\end{array}$ & $\begin{array}{c}\text { Latent heat } \\
(\mathbf{k J} / \mathbf{k g})\end{array}$ & Reference \\
\hline \multirow{5}{*}{$\begin{array}{l}n \text {-Icosane }\left(\mathrm{C}_{20} \mathrm{H}_{42}\right) \\
\mathrm{CH}_{3}\left(\mathrm{CH}_{2}\right)_{18} \mathrm{CH}_{3}\end{array}$} & 36.4 & $788 / .6$ & & & & (47) \\
\hline & 36.6 & $/ 788$ & & & 247 & $(1,52)$ \\
\hline & 36.7 & & & & 246 & $(10)$ \\
\hline & 37.8 & & & & 218.5 & (55) \\
\hline & 38 & 779 & & & 283 & (48) \\
\hline \multirow{2}{*}{$\begin{array}{l}\text { n-Heneicosane }\left(\mathrm{C}_{21} \mathrm{H}_{44}\right) \\
\mathrm{CH}_{3}\left(\mathrm{CH}_{2}\right)_{19} \mathrm{CH}_{3}\end{array}$} & 40.2 & & & & 200 & (10) \\
\hline & 40.2 & $/ 791$ & & & 213 & $(1,52)$ \\
\hline \multirow{3}{*}{$\begin{array}{l}n \text {-Docosane }\left(\mathrm{C}_{22} \mathrm{H}_{46}\right) \\
\mathrm{CH}_{3}\left(\mathrm{CH}_{2}\right)_{20} \mathrm{CH}_{3}\end{array}$} & 41.6 & 785 & 0.22 & $1.93 / 2.38$ & 194.6 & $(56)$ \\
\hline & 44.0 & & & & 249 & (10) \\
\hline & 44.0 & /794 & & & 249 & $(1,52)$ \\
\hline \multirow{2}{*}{$\begin{array}{l}n \text {-Tricosane }\left(\mathrm{C}_{23} \mathrm{H}_{48}\right) \\
\mathrm{CH}_{3}\left(\mathrm{CH}_{2}\right)_{21} \mathrm{CH}_{3}\end{array}$} & 47.5 & & & & 232 & $(10)$ \\
\hline & 47.5 & 1796 & & & 234 & $(1,52)$ \\
\hline \multirow{2}{*}{$\begin{array}{l}n \text {-Tetracosane }\left(\mathrm{C}_{24} \mathrm{H}_{50}\right) \\
\mathrm{CH}_{3}\left(\mathrm{CH}_{2}\right)_{22} \mathrm{CH}_{3}\end{array}$} & 50.6 & & & & 255 & $(10)$ \\
\hline & 50.6 & /799 & & & 255 & $(1,52)$ \\
\hline \multirow{2}{*}{$\begin{array}{l}n \text {-Pentacosane }\left(\mathrm{C}_{25} \mathrm{H}_{52}\right) \\
\mathrm{CH}_{3}\left(\mathrm{CH}_{2}\right)_{23} \mathrm{CH}_{3}\end{array}$} & 49.4 & & & & 238 & $(10)$ \\
\hline & 53.5 & $/ 801$ & & & 238 & $(1,52)$ \\
\hline \multirow{2}{*}{$\begin{array}{l}n \text {-Hexacosane }\left(\mathrm{C}_{26} \mathrm{H}_{54}\right) \\
\mathrm{CH}_{3}\left(\mathrm{CH}_{2}\right)_{24} \mathrm{CH}_{3}\end{array}$} & 56.3 & & & & 256 & $(10)$ \\
\hline & 56.3 & $/ 803$ & & & 256 & $(1,52)$ \\
\hline \multirow{2}{*}{$\begin{array}{l}n \text {-Heptacosane }\left(\mathrm{C}_{27} \mathrm{H}_{56}\right) \\
\mathrm{CH}_{3}\left(\mathrm{CH}_{2}\right)_{25} \mathrm{CH}_{3}\end{array}$} & 58.8 & & & & 236 & $(10)$ \\
\hline & 58.8 & $/ 779$ & & & 235 & $(1,52)$ \\
\hline \multirow{2}{*}{$\begin{array}{l}n \text {-Octacosane }\left(\mathrm{C}_{28} \mathrm{H}_{58}\right) \\
\mathrm{CH}_{3}\left(\mathrm{CH}_{2}\right)_{26} \mathrm{CH}_{3}\end{array}$} & 41.2 & 1806 & & & 254 & $(1,52)$ \\
\hline & 61.6 & & & & 253 & $(10)$ \\
\hline \multirow{2}{*}{$\begin{array}{l}n \text {-Nonacosane }\left(\mathrm{C}_{29} \mathrm{H}_{60}\right) \\
\mathrm{CH}_{3}\left(\mathrm{CH}_{2}\right)_{27} \mathrm{CH}_{3}\end{array}$} & 63.4 & & & & 240 & (10) \\
\hline & 63.4 & /808 & & & 239 & $(1,52)$ \\
\hline \multirow{4}{*}{$\begin{array}{l}n \text {-Triacontane }\left(\mathrm{C}_{30} \mathrm{H}_{62}\right) \\
\mathrm{CH}_{3}\left(\mathrm{CH}_{2}\right)_{28} \mathrm{CH}_{3}\end{array}$} & 65.4 & 1775 & & & 252 & $(1,52)$ \\
\hline & 65.4 & & & & 251 & $(10)$ \\
\hline & 65.8 & $809.6 /$ & & & & (47) \\
\hline & 66 & 775 & & & & (48) \\
\hline $\begin{array}{l}n \text {-Hentriacontane }\left(\mathrm{C}_{31} \mathrm{H}_{64}\right) \\
\mathrm{CH}_{3}\left(\mathrm{CH}_{2}\right)_{29} \mathrm{CH}_{3}\end{array}$ & 68.0 & & & & 242 & (10) \\
\hline $\begin{array}{l}n \text {-Dotriacontane }\left(\mathrm{C}_{32} \mathrm{H}_{66}\right) \\
\mathrm{CH}_{3}\left(\mathrm{CH}_{2}\right)_{30} \mathrm{CH}_{3}\end{array}$ & 69.5 & & & & 170 & (10) \\
\hline $\begin{array}{l}n \text {-Tritriacontane }\left(\mathrm{C}_{33} \mathrm{H}_{68}\right) \\
\mathrm{CH}_{3}\left(\mathrm{CH}_{2}\right)_{31} \mathrm{CH}_{3}\end{array}$ & 73.9 & & & & 268 & (10) \\
\hline $\begin{array}{l}n \text {-Tetratriacontane }\left(\mathrm{C}_{34} \mathrm{H}_{70}\right) \\
\mathrm{CH}_{3}\left(\mathrm{CH}_{2}\right)_{32} \mathrm{CH}_{3}\end{array}$ & 75.9 & & & & 269 & (10) \\
\hline \multirow{2}{*}{$\begin{array}{l}\text { n-Tetracontane }\left(\mathrm{C}_{40} \mathrm{H}_{82}\right) \\
\mathrm{CH}_{3}\left(\mathrm{CH}_{2}\right)_{38} \mathrm{CH}_{3}\end{array}$} & 81.5 & $820.4 /$ & & & & (47) \\
\hline & 82 & & & & & (48) \\
\hline $\begin{array}{l}n \text {-Pentacontane }\left(\mathrm{C}_{50} \mathrm{H}_{102}\right) \\
\mathrm{CH}_{3}\left(\mathrm{CH}_{2}\right)_{48} \mathrm{CH}_{3}\end{array}$ & 95 & 779 & & & & (48) \\
\hline
\end{tabular}

The melting point of paraffins increases with the number of alkane chains within the molecule $(10,38)$, being $n$-Tetradecane the shortest n-alkane, which melts above $0{ }^{\circ} \mathrm{C}$. Alkanes of different chain length are also prepared in different mixtures to get PCMs with different melting temperatures (48). Table 8 shows examples of mixtures of some alkanes.
The undesirable low thermal conductivity of paraffins and other organic PCMs has been overcome through different solutions such us improved heat transfer by means of metal matrix structures or metallic fins $(28,58,59)$, and the development of composite materials based on metal shavings or metal powder embedded in pure PCMs $(37,60)$. 
TABLE 8. Organic PCMs: Mixture of straight-chain alkanes. Properties (solid/liquid)

\begin{tabular}{|c|c|c|c|c|c|c|}
\hline Material $^{\mathrm{a}}$ & $\begin{array}{l}\text { Melting } \\
\text { temp. }\left({ }^{\circ} \mathbf{C}\right)\end{array}$ & $\begin{array}{l}\text { Density } \\
\left(\mathrm{kg} / \mathrm{m}^{3}\right)\end{array}$ & $\begin{array}{c}\text { Thermal conductivity } \\
(\mathrm{W} / \mathrm{m} \mathrm{K})\end{array}$ & $\begin{array}{c}\text { Specific heat } \\
(\mathrm{kJ} / \mathrm{kg} \mathrm{K})\end{array}$ & $\begin{array}{l}\text { Latent heat } \\
(\mathbf{k J} / \mathbf{k g})\end{array}$ & Reference \\
\hline Paraffin C15-C16 & 8 & & & & 153 & $(4,46)$ \\
\hline Paraffin C16-C18 & $19.5-22.2$ & & & & 152 & $(57)$ \\
\hline Paraffin C13-C24 & $22-24$ & $900 / 760$ & $0.21 /$ & $/ 2.1$ & 189 & $(4,15,46)$ \\
\hline Paraffin C16-C28 & $42-44$ & $910 / 765$ & $0.21 /$ & $/ 2.1$ & 189 & $(4,46)$ \\
\hline Paraffin $\mathrm{C} 20-\mathrm{C} 33$ & $48-50$ & $912 / 769$ & $0.21 /$ & $/ 2.1$ & 189 & $(4,46)$ \\
\hline Paraffin C22-C45 & $58-60$ & $920 / 795$ & $0.21 /$ & $/ 2.1$ & 189 & (4) \\
\hline Paraffin C23-C45 & $62-64$ & $915 / 790$ & $0.21 /$ & $/ 2.1$ & 189 & (4) \\
\hline Paraffin C21-C50 & $66-68$ & $930 / 830$ & $0.21 /$ & $/ 2.1$ & 189 & $(4,46)$ \\
\hline
\end{tabular}

${ }^{a}$ Paraffin nomenclature $(\mathrm{Cn})$ refers to the number of n-alkanes (straight-chain alkanes) in each compound. For instance, Paraffin $\mathrm{C} 15$ consists of n-Pentadecane with the formula $\mathrm{C}_{15} \mathrm{H}_{32}$ or $\mathrm{CH}_{3}-\left(\mathrm{CH}_{2}\right)_{13}-\mathrm{CH}_{3}$.

4.2.1.2. Fattyacids Along with parrafins, saturated and unsaturated fatty acids have been widely tested and used as PCMs. Saturated fatty acids are characterized by the general formula $\mathrm{CH}_{3}\left(\mathrm{CH}_{2}\right)_{2 \mathrm{n}} \mathrm{COOH}$ (48). Fatty acids show reproducible melting and freezing behaviour with little or no subcooling (4) and are stable upon cycling since they consist of only one component without the possibility of phase separation (48). Saturated fatty acids also exhibit low phase transition volume changes (21). On the other hand, they show some drawbacks such as low thermal conductivity, mild corrosiveness, and higher cost than technical grade paraffins (approximately 2-2.5 times greater) $(10,48)$.

Tables 9 and 10 show the tested properties of saturated and unsaturated fatty acids used as LHSMs.

Their melting temperature generally increases with the length of the molecule $(1,48)$. Different fatty acids can also be mixed to design PCMs with melting temperatures differing from those of the pure fatty acids (48).

Sari and Kaygusuz (66) studied the thermal reliability under 910 thermal cycles of stearic acid, palmitic acid, myristic acid and lauric acid. The percentage of latent heat losses after cycling was $31.9 \%$, $17.8 \%, 17.1 \%$ and $37.2 \%$, respectively, indicating that palmitic acid and myristic acid are more suitable for latent heat storage.

4.2.1.3. Fatty acid esters Fatty acid esters are derived from acids in which one hydroxyl $(-\mathrm{OH})$ group is replaced by one alkyl (-O) group (21). They show a narrow temperature range of phase transition and their mixtures can form eutectics with negligible subcooling (1). They are widely available due to their commercial use in other industries (polymer, cosmetics, clothes, etc.) (21). Table 11 shows the tested properties of fatty acid esters used as LHSMs.

4.2.1.4. Sugar alcohols Different sugar alcohols and polyalcohols have been tested as potential
PCMs. They are characterized by the general formula $\mathrm{HOCH}_{2}(\mathrm{CHOH})_{n} \mathrm{CH}_{2} \mathrm{OH}$. Sugar alcohols have much higher latent heat of fusion than other organic materials (approximately $300 \mathrm{~kJ} / \mathrm{kg}$ and $375 \mathrm{MJ} / \mathrm{m}^{3}$ ). However, they have a high melting temperature $\left(>90^{\circ} \mathrm{C}\right)$ and may be combustive, which limits their application in buildings.

Table 12 shows the tested properties of sugar alcohols used as LHSMs.

4.2.1.5. Polyethylene glycols (PEGs) Polyethylene glycols (PEGs) are composed of dimethyl ether chains having the hydroxyl group at the end, being characterized by the chemical formula $\mathrm{HO}-\mathrm{CH}_{2}-$ $\left(\mathrm{CH}_{2}-\mathrm{O}-\mathrm{CH}_{2}-\right)_{\mathrm{n}}-\mathrm{CH}_{2}-\mathrm{OH}$ or $\mathrm{C}_{2 \mathrm{n}} \mathrm{H}_{4 \mathrm{n}+2} \mathrm{O}_{\mathrm{n}+1}$ (21). They are chemically and thermally stable under cycling, non-flammable, non-toxic, non-corrosive and have moderate cost. However, they present low thermal conductivity as other organic materials. Table 13 shows the tested properties of PEGs used as LHSMs. The melting point and latent heat of fusion of PEGs increase with increasing molecular weight (1).

4.2.1.6. Other compounds Other organic compounds tested as PCMs are aromatic hydrocarbons, monohydroxy alcohols, ketones, ethers, halogen derivatives, sulphur compounds, amides, dienes, oleochemical carbonates (Table 14). They show a relatively moderate thermal storage density (approximately $100-200 \mathrm{~kJ} / \mathrm{kg}$ and $100-200 \mathrm{MJ} / \mathrm{m}^{3}$ ). Most of them have not been studied in detail regarding their applicability as PCMs but could be a valuable alternative to paraffins and salt hydrates, which currently dominate the PCM market.

\subsubsection{Inorganics}

Inorganic materials have been widely tested and implemented in building applications as PCMs. They usually have similar latent heat of fusion per 
TABLE 9. Organic PCMs: Saturated fatty acids. Properties (solid/liquid)

\begin{tabular}{|c|c|c|c|c|c|c|}
\hline Material & $\begin{array}{l}\text { Melting } \\
\text { temp. }\left({ }^{\circ} \mathbf{C}\right)\end{array}$ & $\begin{array}{l}\text { Density } \\
\left(\mathrm{kg} / \mathrm{m}^{3}\right)\end{array}$ & $\begin{array}{c}\text { Thermal conductivity } \\
(\mathrm{W} / \mathrm{m} \mathrm{K})\end{array}$ & $\begin{array}{c}\text { Specific heat } \\
(\mathrm{kJ} / \mathrm{kg} \mathrm{K})\end{array}$ & $\begin{array}{c}\text { Latent heat } \\
(\mathrm{kJ} / \mathrm{kg})\end{array}$ & Reference \\
\hline \multirow{2}{*}{$\begin{array}{l}\text { Caprylic acid } \\
\mathrm{CH}_{3}\left(\mathrm{CH}_{2}\right)_{6} \mathrm{COOH}\end{array}$} & 16 & $981 / 901$ & $/ 0.149$ & & & $(5,46,61)$ \\
\hline & 16.5 & $1033 / 862$ & $/ 0.148$ & & 149 & (4) \\
\hline \multirow{7}{*}{$\begin{array}{l}\text { Capric acid } \\
\mathrm{CH}_{3}\left(\mathrm{CH}_{2}\right)_{8} \mathrm{COOH}\end{array}$} & 28.8 & & & & 147.4 & $(62)$ \\
\hline & 31.04 & & & & 190.21 & $\begin{array}{l}(63) \\
(22)\end{array}$ \\
\hline & 31.3 & & & & 163 & (44) \\
\hline & 31.5 & 1886 & $/ 0.149$ & & 153 & (4) \\
\hline & 32 & $1004 / 878$ & $/ 0.153$ & & 152.7 & $(5,46,61)$ \\
\hline & 32.14 & & & & 154.24 & (64) \\
\hline & 35.6 & & & & 169.9 & $(65)$ \\
\hline \multirow{8}{*}{$\begin{array}{l}\text { Lauric acid } \\
\mathrm{CH}_{3}\left(\mathrm{CH}_{2}\right)_{10} \mathrm{COOH}\end{array}$} & $40-43$ & & & & $167-171$ & (44) \\
\hline & 42.0 & & & & 123.9 & $(62)$ \\
\hline & $42-44$ & 1870 & $/ 0.147$ & 1.6 & 178 & (4) \\
\hline & 42.6 & & & $1.7 / 2.3$ & 211.6 & (66) \\
\hline & 42.6 & & & & 176.6 & $(67)$ \\
\hline & 43.97 & & & & 200.18 & $(22,63)$ \\
\hline & 44 & $1007 / 862$ & & & 177.4 & $(5,46)$ \\
\hline & 48.5 & & & & 187.7 & $(65)$ \\
\hline \multirow{8}{*}{$\begin{array}{l}\text { Myristic acid } \\
\mathrm{CH}_{3}\left(\mathrm{CH}_{2}\right)_{12} \mathrm{COOH}\end{array}$} & $50.4-53.6$ & & & & 189 & (44) \\
\hline & 51.5 & & & $2.8 / 2.42$ & 204.5 & $(68)$ \\
\hline & 52.2 & & & & 182.6 & (67) \\
\hline & 53.52 & & & & 201.65 & $(22,63)$ \\
\hline & 53.8 & & & $1.7 / 2.4$ & 192.0 & (66) \\
\hline & 54 & 1844 & & $1.6 / 2.7$ & 187 & (4) \\
\hline & 58 & $990 / 861$ & & & 186.6 & $(5,46)$ \\
\hline & 61.0 & & & & 201.0 & $(65)$ \\
\hline \multirow{8}{*}{$\begin{array}{l}\text { Palmitic acid } \\
\mathrm{CH}_{3}\left(\mathrm{CH}_{2}\right)_{14} \mathrm{COOH}\end{array}$} & $57.8-61.8$ & & & $2.20 / 1.70$ & 201 & (44) \\
\hline & 59.40 & & & & 218.53 & (64) \\
\hline & 59.9 & & & $1.9 / 2.8$ & 197.9 & (66) \\
\hline & 61 & $942 / 862$ & 0.16 & $2.2 / 2.48$ & 203.4 & (69) \\
\hline & 62.6 & & & & 208.5 & $(22,70)$ \\
\hline & 63 & 1847 & $/ 0.165$ & & 187 & (4) \\
\hline & 64 & $989 / 850$ & $/ 0.162$ & & 185.4 & $(5,46,61)$ \\
\hline & 66.8 & & & & 216.5 & (65) \\
\hline \multirow{6}{*}{$\begin{array}{l}\text { Stearic acid } \\
\mathrm{CH}_{3}\left(\mathrm{CH}_{2}\right)_{16} \mathrm{COOH}\end{array}$} & 53.6 & & & $1.6 / 2.2$ & 174.6 & (66) \\
\hline & 60-61 & $1080 / 1150$ & 0.18 & $2.83 / 2.38$ & 186.5 & (71) \\
\hline & $65.2-68.5$ & & & $2.07 / 1.90$ & 210 & (44) \\
\hline & 69 & $965 / 848$ & & & 202.5 & $(5,46,61)$ \\
\hline & 70 & 941/ & $/ 0.172$ & $/ 2.35$ & 203 & (4) \\
\hline & 72.5 & & & & 220.4 & $(65)$ \\
\hline $\begin{array}{l}\text { Arachidic acid } \\
\mathrm{CH}_{3}\left(\mathrm{CH}_{2}\right)_{18} \mathrm{COOH}\end{array}$ & 74.0 & & & & 227 & (1) \\
\hline
\end{tabular}

mass than organic materials, but higher ones per volume due to their high density (48). Main inorganic materials used as PCMs are salt hydrates and metals.
4.2.2.1. Salt hydrates Salt hydrates are characterized by the general formula: $\mathrm{AB} \cdot \mathrm{xH}_{2} \mathrm{O}$, being inorganic salts containing water of crystallization (21). Salt hydrates have higher LHSC and thermal 
TABLE 10. Organic PCMs: Unsaturated fatty acids. Properties (solid/liquid)

\begin{tabular}{|c|c|c|c|c|c|c|}
\hline Material & $\begin{array}{l}\text { Melting } \\
\text { temp. }\left({ }^{\circ} \mathrm{C}\right)\end{array}$ & $\begin{array}{l}\text { Density } \\
\left(\mathrm{kg} / \mathrm{m}^{3}\right)\end{array}$ & $\begin{array}{c}\text { Thermal conductivity } \\
(\mathrm{W} / \mathrm{m} \mathrm{K})\end{array}$ & $\begin{array}{l}\text { Specific heat } \\
(\mathrm{kJ} / \mathrm{kg} \mathrm{K})\end{array}$ & $\begin{array}{l}\text { Latent heat } \\
(\mathbf{k J} / \mathbf{k g})\end{array}$ & Reference \\
\hline $\begin{array}{l}\text { Undecylenic acid } \\
\mathrm{CH}_{2}=\mathrm{CH}\left(\mathrm{CH}_{2}\right)_{8} \mathrm{COOH}\end{array}$ & 24.6 & & & & 141 & $(1,72)$ \\
\hline $\begin{array}{l}\text { Eladic acid } \\
\mathrm{C}_{8} \mathrm{H}_{7} \mathrm{C} 9 \mathrm{H}_{16} \mathrm{COOH}\end{array}$ & 47 & & & & 218 & (10) \\
\hline
\end{tabular}

TAble 11. Organic PCMs: Fatty acid esters. Properties (solid/liquid)

\begin{tabular}{|c|c|c|c|c|c|c|}
\hline Material & $\begin{array}{l}\text { Melting } \\
\text { temp. }\left({ }^{\circ} \mathbf{C}\right)\end{array}$ & $\begin{array}{l}\text { Density } \\
\left(\mathrm{kg} / \mathrm{m}^{3}\right)\end{array}$ & $\begin{array}{l}\text { Thermal conductivity } \\
\text { (W/m K) }\end{array}$ & $\begin{array}{l}\text { Specific heat } \\
(\mathrm{kJ} / \mathrm{kg} \mathrm{K})\end{array}$ & $\begin{array}{l}\text { Latent heat } \\
(\mathbf{k J} / \mathbf{k g})\end{array}$ & Reference \\
\hline $\begin{array}{l}\text { Isopropyl palmitate }\left(\mathrm{C}_{19} \mathrm{H}_{38} \mathrm{O}_{2}\right) \\
\mathrm{CH}_{3}\left(\mathrm{CH}_{2}\right)_{14} \mathrm{COOCH}\left(\mathrm{CH}_{3}\right)_{2}\end{array}$ & 11 & & & & 100 & $(46,73,74)$ \\
\hline $\begin{array}{l}\text { Isopropyl stearate }\left(\mathrm{C}_{21} \mathrm{H}_{42} \mathrm{O}_{2}\right) \\
\mathrm{CH}_{3}\left(\mathrm{CH}_{2}\right)_{16} \mathrm{COOCH}\left(\mathrm{CH}_{3}\right)_{2}\end{array}$ & 14 & & & & 142 & $(46,73,74)$ \\
\hline \multirow{2}{*}{$\begin{array}{l}\text { Propyl palmitate }\left(\mathrm{C}_{17} \mathrm{H}_{34} \mathrm{O}_{2}\right) \\
\mathrm{CH}_{3}\left(\mathrm{CH}_{2}\right)_{12} \mathrm{COOC}_{3} \mathrm{H}_{7}\end{array}$} & $16-19$ & & & & 186 & $(1,75)$ \\
\hline & 19 & & & & 186 & $(46,76)$ \\
\hline \multirow{4}{*}{$\begin{array}{l}\text { Butyl stearate }\left(\mathrm{C}_{22} \mathrm{H}_{44} \mathrm{O}_{2}\right) \\
\mathrm{CH}_{3}\left(\mathrm{CH}_{2}\right)_{16} \mathrm{COO}\left(\mathrm{CH}_{2}\right)_{3} \mathrm{CH}_{3}\end{array}$} & $18-23$ & & & & 140 & (75) \\
\hline & 19 & & & & 200 & $(46,73,74)$ \\
\hline & 19 & & & & 140 & (76) \\
\hline & 23 & & & & 140 & $(22)$ \\
\hline Allyl palmitate $\left(\mathrm{C}_{19} \mathrm{H}_{36} \mathrm{O}_{2}\right)$ & 22.6 & & & & 173 & $(1,77)$ \\
\hline $\begin{array}{l}\text { Dimethyl sebacate }\left(\mathrm{C}_{12} \mathrm{H}_{22} \mathrm{O}_{4}\right) \\
\left(\mathrm{CH}_{2}\right)_{8}\left(\mathrm{COOCH}_{3}\right)_{2}\end{array}$ & 21 & & & & 135 & $(46,73,74)$ \\
\hline $\begin{array}{l}\text { Methyl palmitate }\left(\mathrm{C}_{17} \mathrm{H}_{34} \mathrm{O}_{2}\right) \\
\mathrm{CH}_{3}\left(\mathrm{CH}_{2}\right)_{14} \mathrm{CO}_{2} \mathrm{CH}_{3}\end{array}$ & 28.8 & & & & 163.2 & $(1,62)$ \\
\hline $\begin{array}{l}\text { Vinyl stearate }\left(\mathrm{C}_{20} \mathrm{H}_{38} \mathrm{O}_{2}\right) \\
\mathrm{CH}_{3}\left(\mathrm{CH}_{2}\right)_{16} \mathrm{COOCH}=\mathrm{CH}_{2}\end{array}$ & $27-29$ & & & & 122 & $(46,73,74)$ \\
\hline $\begin{array}{l}\text { Methyl stearate }\left(\mathrm{C}_{19} \mathrm{H}_{38} \mathrm{O}_{2}\right) \\
\mathrm{CH}_{3}\left(\mathrm{CH}_{2}\right)_{16} \mathrm{CO}_{2} \mathrm{CH}_{3}\end{array}$ & 37.0 & & & & 160.7 & $(1,62)$ \\
\hline $\begin{array}{l}\text { Methyl-12 hydroxy-stearate }\left(\mathrm{C}_{19} \mathrm{H}_{38} \mathrm{O}_{3}\right) \\
\mathrm{CH}_{3}\left(\mathrm{CH}_{2}\right)_{10} \mathrm{CH}\left(\mathrm{CH}_{2}\right)_{5} \mathrm{COOCH}_{3} \mathrm{OH}\end{array}$ & 43 & & & & 126 & $(46,73,74)$ \\
\hline Ethylene glycol distearate $\left(\mathrm{C}_{38} \mathrm{H}_{74} \mathrm{O}_{4}\right)$ & 65.5 & 972.9 & & & 215.8 & $(1,78)$ \\
\hline
\end{tabular}

TABle 12. Organic PCMs: Sugar alcohols. Properties (solid/liquid)

\begin{tabular}{|c|c|c|c|c|c|c|}
\hline Material & $\begin{array}{l}\text { Melting } \\
\text { temp. }\left({ }^{\circ} \mathbf{C}\right)\end{array}$ & $\begin{array}{l}\text { Density } \\
\left(\mathrm{kg} / \mathrm{m}^{3}\right)\end{array}$ & $\begin{array}{l}\text { Thermal conductivity } \\
\text { (W/m K) }\end{array}$ & $\begin{array}{l}\text { Specific heat } \\
(\text { kJ/kg K) }\end{array}$ & $\begin{array}{l}\text { Latent heat } \\
(\mathbf{k J} / \mathbf{k g})\end{array}$ & Reference \\
\hline \multirow[t]{3}{*}{$\begin{array}{l}\text { Xylitol }\left(\mathrm{C}_{5} \mathrm{H}_{7}(\mathrm{OH})_{5}\right) \\
\mathrm{HOCH}_{2}\left[\mathrm{CH}(\mathrm{OH}]_{3} \mathrm{CH}_{2} \mathrm{OH}\right.\end{array}$} & 93 & & & & 248.7 & $\begin{array}{l}(79) \\
(22)\end{array}$ \\
\hline & 93 & & & & 280 & (80) \\
\hline & 94 & $1500 /$ & & & 263 & (48) \\
\hline \multirow{2}{*}{$\begin{array}{l}\text { D-Sorbitol }\left(\mathrm{C}_{6} \mathrm{H}_{8}(\mathrm{OH})_{6}\right) \\
\mathrm{HOCH}_{2}[\mathrm{CH}(\mathrm{OH})]_{4} \mathrm{CH}_{2} \mathrm{OH}\end{array}$} & 97 & $1520 /$ & & & 185 & $(48)$ \\
\hline & 97 & & & & 110 & (80) \\
\hline \multirow{3}{*}{$\begin{array}{l}\text { Erythritol }\left(\mathrm{C}_{4} \mathrm{H}_{6}(\mathrm{OH})_{4}\right) \\
\mathrm{HOCH}_{2}[\mathrm{CH}(\mathrm{OH})]_{2} \mathrm{CH}_{2} \mathrm{OH}\end{array}$} & 117 & & & & 344 & (80) \\
\hline & 118.0 & $1480 / 1300$ & $0.733 / 0.326$ & & 339.8 & $(46,81)$ \\
\hline & 120 & $1480 / 1300$ & $0.733 / 0.326$ & & 340 & $(48)$ \\
\hline $\begin{array}{l}\text { Mannitol }\left(\mathrm{C}_{6} \mathrm{H}_{14} \mathrm{O}_{6}\right) \\
\mathrm{HOCH}_{2}[\mathrm{CH}(\mathrm{OH})]_{4} \mathrm{CH}_{2} \mathrm{OH}\end{array}$ & 165 & & & & 341 & $(80)$ \\
\hline
\end{tabular}


TABLe 13. Organic PCMs: Polyethylene glycols (PEG). Properties (solid/liquid)

\begin{tabular}{|c|c|c|c|c|c|c|}
\hline Material & $\begin{array}{l}\text { Melting } \\
\text { temp. }\left({ }^{\circ} \mathbf{C}\right)\end{array}$ & $\begin{array}{l}\text { Density } \\
\left(\mathrm{kg} / \mathrm{m}^{3}\right)\end{array}$ & $\begin{array}{c}\text { Thermal conductivity } \\
(\mathrm{W} / \mathrm{m} \mathrm{K})\end{array}$ & $\begin{array}{l}\text { Specific heat } \\
(\mathrm{kJ} / \mathrm{kg} \mathrm{K})\end{array}$ & $\begin{array}{c}\text { Latent heat } \\
(\mathrm{kJ} / \mathrm{kg})\end{array}$ & Reference \\
\hline $\begin{array}{l}\text { Diethylene glycol } \\
\mathrm{C}_{4} \mathrm{H}_{10} \mathrm{O}_{3}\end{array}$ & -10 to -7 & $/ 1120$ & & & & $(48)$ \\
\hline $\begin{array}{l}\text { Triethylene glycol } \\
\mathrm{C}_{6} \mathrm{H}_{14} \mathrm{O} 4\end{array}$ & -7 & $/ 1120$ & & & & $(48)$ \\
\hline \multirow[t]{2}{*}{ PEG 400} & 4.2 & & & & 117.6 & (1) \\
\hline & 8 & $1228 / 1125$ & $/ 0.187$ & - & 99.6 & $(5,46,61)$ \\
\hline \multirow[t]{2}{*}{ PEG 600} & 12.5 & & & & 129.1 & $(1,49)$ \\
\hline & 22 & $1232 / 1126$ & $/ 0.189$ & - & 127.2 & $(5,46,61)$ \\
\hline \multirow[t]{2}{*}{ PEG 1000} & $35-40$ & & & & & $(48)$ \\
\hline & 40.0 & & & & 168.6 & $(1,82)$ \\
\hline PEG 3000 & $52-56$ & & & & & $(48)$ \\
\hline PEG 3400 & 63.4 & & & & 166.8 & $(1,65)$ \\
\hline PEG 6000 & 66 & $1212 / 1085$ & - & - & 190.0 & $(5,46,61)$ \\
\hline \multirow[t]{2}{*}{ PEG 10000} & $55-60$ & & & & & (48) \\
\hline & 65.9 & & & & 171.6 & $(1,65)$ \\
\hline PEG 20000 & 67.7 & & & & 160.2 & $(1,65)$ \\
\hline PEG 35000 & 68.7 & & & & 166.9 & $(1,65)$ \\
\hline PEG 100000 & 67.0 & & & & 175.8 & (1) \\
\hline PEG 1000000 & 70.0 & & & & 174.0 & (1) \\
\hline
\end{tabular}

TABle 14. Organic PCMs: Other compounds. Properties (solid/liquid)

\begin{tabular}{|c|c|c|c|c|c|c|c|}
\hline $\begin{array}{l}\text { Class of } \\
\text { compounds }\end{array}$ & Material & $\begin{array}{l}\text { Melting } \\
\text { temp. }\left({ }^{\circ} \mathbf{C}\right)\end{array}$ & $\begin{array}{l}\text { Density } \\
\left(\mathrm{kg} / \mathrm{m}^{3}\right)\end{array}$ & $\begin{array}{c}\text { Thermal } \\
\text { conductivity } \\
(\mathrm{W} / \mathrm{m} \mathrm{K})\end{array}$ & $\begin{array}{l}\text { Specific heat } \\
(\mathrm{kJ} / \mathrm{kg} \mathrm{K})\end{array}$ & $\begin{array}{l}\text { Latent heat } \\
(\mathrm{kJ} / \mathrm{kg})\end{array}$ & Reference \\
\hline \multirow[t]{2}{*}{$\begin{array}{l}\text { Aromatic } \\
\text { hydrocarbons }\end{array}$} & $\begin{array}{l}\text { Biphenyl } \\
\left(\mathrm{C}_{12} \mathrm{H}_{10}\right)_{2}\end{array}$ & 71 & $1166 / 991$ & & & 119.2 & $(5,46,61)$ \\
\hline & $\begin{array}{l}\text { Naphthalene } \\
\mathrm{C}_{10} \mathrm{H}_{8}\end{array}$ & 80 & $1145 / 976$ & $0.310 / 0.132$ & $2.8 /$ & 147.7 & $(5,46,61)$ \\
\hline \multirow{5}{*}{$\begin{array}{l}\text { Monohydroxy } \\
\text { alcohols }\end{array}$} & 1-dodecanol $\left(\mathrm{C}_{12} \mathrm{H}_{26} \mathrm{O}\right)$ & $17.5-23.3$ & & & & 188.8 & $(75)$ \\
\hline & $\mathrm{CH}_{3}\left(\mathrm{CH}_{2}\right)_{11} \mathrm{OH}$ & 23.8 & & & & 184 & (77) \\
\hline & & 26 & & & & 200 & $(46,76)$ \\
\hline & 1-Tetradecanol $\left(\mathrm{C}_{14} \mathrm{H}_{30} \mathrm{O}\right)$ & 38 & & & & 205 & $(46,76)$ \\
\hline & $\mathrm{CH}_{3}\left(\mathrm{CH}_{2}\right)_{12} \mathrm{OH}$ & 39.3 & & & & 221.23 & $(83)$ \\
\hline \multirow[t]{2}{*}{ Ketones } & $\begin{array}{l}\text { Trimethylcyclohexene-1, 4-dione } \\
\left(\mathrm{C}_{9} \mathrm{H}_{12} \mathrm{O}_{2}\right)\end{array}$ & 24.1 & & & & 47 & $(72)$ \\
\hline & $\begin{array}{l}\text { Phorone }\left(\mathrm{C}_{9} \mathrm{H}_{14} \mathrm{O}\right) \\
\left(\mathrm{CH}_{3}\right)_{2} \mathrm{C}=\mathrm{CHCOCH}=\mathrm{C}\left(\mathrm{CH}_{3}\right)_{2}\end{array}$ & 25.8 & & & & 124 & $(72)$ \\
\hline Ethers & $\begin{array}{l}\text { Diphenyl ether } \\
\left(\mathrm{C}_{6} \mathrm{H}_{5}\right)_{2} \mathrm{O}\end{array}$ & 27.2 & & & & 97 & $(1,72)$ \\
\hline \multirow[t]{3}{*}{$\begin{array}{l}\text { Halogen } \\
\text { derivative }\end{array}$} & $\begin{array}{l}\text { Chlorobenzothiazole } \\
\mathrm{C}_{7} \mathrm{H}_{4} \mathrm{ClNS}\end{array}$ & 18.6 & & & & 65 & $(1,72)$ \\
\hline & 1-Iodehexadecane & 22.2 & & & & 131 & $(1,72)$ \\
\hline & $\begin{array}{l}\text { 3-Iodoaniline } \\
\mathrm{IC}_{6} \mathrm{H}_{4} \mathrm{NH}_{2}\end{array}$ & 22.5 & & & & 64 & $(1,72)$ \\
\hline \multirow[t]{2}{*}{$\begin{array}{l}\text { Sulphur } \\
\text { compounds }\end{array}$} & $\begin{array}{l}\text { Dimethyl sulfoxide } \\
\left(\mathrm{CH}_{3}\right)_{2} \mathrm{SO}\end{array}$ & 16.5 & $1009 / 1009$ & & $1.45 / 1.88$ & 85.7 & $(46,84)$ \\
\hline & $\begin{array}{l}\text { Octadecyl 3-mercaptopropionate } \\
\text { HS- } \mathrm{CH}_{2} \mathrm{CH}_{2} \mathrm{COO}\left(\mathrm{C}_{18} \mathrm{H}_{37}\right)\end{array}$ & 21 & & & & 141 & $(73,74)$ \\
\hline
\end{tabular}


TABle 14. Continued

\begin{tabular}{|c|c|c|c|c|c|c|c|}
\hline $\begin{array}{l}\text { Class of } \\
\text { compounds }\end{array}$ & Material & $\begin{array}{l}\text { Melting } \\
\text { temp. }\left({ }^{\circ} \mathbf{C}\right)\end{array}$ & $\begin{array}{l}\text { Density } \\
\left(\mathrm{kg} / \mathrm{m}^{3}\right)\end{array}$ & $\begin{array}{c}\text { Thermal } \\
\text { conductivity } \\
(\mathrm{W} / \mathrm{m} \mathrm{K})\end{array}$ & $\begin{array}{l}\text { Specific heat } \\
(\mathrm{kJ} / \mathrm{kg} \mathrm{K})\end{array}$ & $\begin{array}{c}\text { Latent heat } \\
(\mathrm{kJ} / \mathrm{kg})\end{array}$ & Reference \\
\hline \multirow{4}{*}{ Amides } & $\begin{array}{l}\text { Octadecyl thioglycolate } \\
\text { HS- } \mathrm{CH}_{2} \mathrm{COO}\left(\mathrm{C}_{18} \mathrm{H}_{37}\right)\end{array}$ & 26 & & & & 91 & $(73,74)$ \\
\hline & $\begin{array}{l}\text { Dilauryl thiodipropionate } \\
\mathrm{S}\left(\mathrm{CH}_{2} \mathrm{CH}_{2} \mathrm{COO}\left(\mathrm{C}_{12} \mathrm{H}_{25}\right)\right)_{2}\end{array}$ & 39 & & & & 159 & $(73,74)$ \\
\hline & $\begin{array}{l}\text { Propionamide }\left(\mathrm{C}_{3} \mathrm{H}_{7} \mathrm{NO}\right) \\
\mathrm{CH}_{3} \mathrm{CH}_{2} \mathrm{CONH}_{2}\end{array}$ & 79 & & & & 168.2 & $(46)$ \\
\hline & $\begin{array}{l}\text { Acetamide } \\
\mathrm{CH}_{3} \mathrm{CONH}_{2}\end{array}$ & 81 & & & & 241 & $(1,10)$ \\
\hline Dienes & Dicyclopentadiene $\left(\mathrm{C}_{10} \mathrm{H}_{12}\right)$ & 28.7 & & & & 11.7 & $(1,49)$ \\
\hline \multirow{5}{*}{$\begin{array}{l}\text { Oleochemical } \\
\text { carbonates }\end{array}$} & Decyl carbonate $\left(\mathrm{C}_{21} \mathrm{H}_{42} \mathrm{O}_{3}\right)$ & -2.2 & & & & 144 & $(1,85)$ \\
\hline & Dodecyl carbonate $\left(\mathrm{C}_{25} \mathrm{H}_{50} \mathrm{O}_{3}\right)$ & 19.3 & & & & 200 & $(1,85)$ \\
\hline & Tetradecyl carbonate $\left(\mathrm{C}_{29} \mathrm{H}_{58} \mathrm{O}_{3}\right)$ & 33.7 & & & & 227 & $(1,85)$ \\
\hline & Hexadecyl carbonate $\left(\mathrm{C}_{33} \mathrm{H}_{66} \mathrm{O}_{3}\right)$ & 44.9 & & & & 219 & $(1,85)$ \\
\hline & Octadecyl carbonate $\left(\mathrm{C}_{37} \mathrm{H}_{74} \mathrm{O}_{3}\right)$ & 51.6 & & & & 223 & $(1,85)$ \\
\hline
\end{tabular}

conductivity than organic materials for low-tomoderate temperature applications, approximately $350 \mathrm{MJ} / \mathrm{m}^{3}$ and $0.5 \mathrm{~W} / \mathrm{m} \mathrm{K}$, respectively $(15,38)$. Also, they are non-flammable and cheaper than paraffins. However, they often present some disadvantages which limit their applicability, such us low thermal reliability for long-operation periods, phase segregation, subcooling and corrosiveness $(2,10,38,44)$. Table 15 shows the properties of the main salt hydrates tested.

Some of the barriers for using salt hydrates can be eliminated or significantly reduced to obtain a long-term thermal cycling material by different techniques. One of the main methods used for preventing segregation and sedimentation is adding thickening agents. The decomposition along the melting process into water and salt with less content of water is mainly due to density difference between salt and water, which leads the lower hydrate (or anhydrous salt) to settle down at the bottom of the container (10). Thus, by adding polymeric or cellulosic gelling materials, such as wooden fibres or attapulgite clays, the lifetime of salt hydrates can be increased up to 1000 cycles without significantly impairing their properties $(21,74)$. Other methods are based on the addition of water to a hydrate, in the quantity which slightly exceeds the stoichiometric ratio, and mechanical stirring of the mixture $(10,74)$. Supercooling, which refers to a liquid existing at a temperature lower the melting temperature, can be reduced or eliminated by using nucleating agents, which accelerate the crystallization of supersaturated solutions, such as borax or carbon (90). However, they often reduce the thermal conductivity (21). A list of nucleating agents used in salt hydrates has been reported by Kenisarin (74).
Regarding the corrosion properties of salt hydrates, according to Kenisarin (74), stainless steel is compatible with all salts and mild steels would react only weakly. However, aluminium, copper and brass are not recommended in applications because of their limited resistance.

4.2.2.2. Metals and metal alloys Some metal compounds have been tested as PCMs for low-tomoderate temperature applications. Most of fusible alloys are based on the combination of bismuth with other metals, such as lead, tin, indium, and cadmium (38). They have high volumetric fusion heat because of their high density (10) and present high thermal conductivity, good thermal stability and reliability, and a negligible volume change during phase change $(1,22,38)$. However, their usage in buildings is constrained by their moderate-high melting temperature, scarce availability and very high cost. Table 16 shows the properties of main metals and metal alloys reported.

\subsubsection{Eutectics}

Eutectics are mixtures of two or more components. Theses mixtures can be developed from organic-organic, organic-inorganic and inorganicinorganic compounds. One of the most important characteristics of eutectics is their capability to change of phase congruently without phase segregation, with a single melting/freezing point (21). Furthermore, the melting temperature of eutectics is usually lower than that of their single compounds (38). A list of eutectic mixtures with a wide temperature range is presented in the following sub-sections. 
TABLE 15. Inorganic PCMs for building applications: Salt hydrates. General formula: $\mathrm{AB} \cdot \mathrm{xH}_{2} \mathrm{O}$. Properties (solid/liquid)

\begin{tabular}{|c|c|c|c|c|c|c|}
\hline Material & $\begin{array}{l}\text { Melting } \\
\text { temp. }\left({ }^{\circ} \mathrm{C}\right)\end{array}$ & $\begin{array}{l}\text { Density } \\
\left(\mathrm{kg} / \mathrm{m}^{3}\right)\end{array}$ & $\begin{array}{l}\text { Thermal conductivity } \\
(\mathrm{W} / \mathrm{m} \mathrm{K})\end{array}$ & $\begin{array}{l}\text { Specific heat } \\
(\mathrm{kJ} / \mathrm{kg} \mathrm{K})\end{array}$ & $\begin{array}{l}\text { Latent heat } \\
(\mathrm{kJ} / \mathrm{kg})\end{array}$ & Reference \\
\hline $\mathrm{H}_{2} \mathrm{O}$ & 0 & $900 / 1000$ & $2.2 / 0.6$ & $2 / 4.19$ & 334 & $(40)$ \\
\hline \multirow{3}{*}{$\begin{array}{l}\text { Lithium chlorate trihydrate } \\
\mathrm{LiClO}_{3} \cdot 3 \mathrm{H}_{2} \mathrm{O}\end{array}$} & 8 & $1720 / 1530$ & & & 155 & (48) \\
\hline & 8.1 & $1720 / 1530$ & & & 253 & $(8,20)$ \\
\hline & 8.1 & & & & & (9) \\
\hline $\begin{array}{l}\text { Zinc chloride tetrahydrate } \\
\mathrm{ZnCl}_{2} \cdot 3 \mathrm{H}_{2} \mathrm{O}\end{array}$ & 10 & & & & & $(46,86)$ \\
\hline $\begin{array}{l}\text { Potassium phosphate hexahydrate } \\
\mathrm{K}_{2} \mathrm{HPO}_{4} \cdot 6 \mathrm{H}_{2} \mathrm{O}\end{array}$ & 13 & & & & & $(46,86)$ \\
\hline \multirow{2}{*}{$\begin{array}{l}\text { Sodium hydroxide } \\
\mathrm{NaOH} \cdot 3 \frac{1}{2} \mathrm{H}_{2} \mathrm{O}\end{array}$} & 15 & & & & & $(46,86)$ \\
\hline & 15.4 & & & & & (9) \\
\hline $\begin{array}{l}\text { Sodium chromate decahydrate } \\
\mathrm{Na}_{2} \mathrm{CrO}_{4} \cdot 10 \mathrm{H}_{2} \mathrm{O}\end{array}$ & 18 & & & & & $(46,86)$ \\
\hline \multirow{2}{*}{$\begin{array}{l}\text { Potassium fluoride tetrahydrate } \\
\mathrm{KF} \cdot 4 \mathrm{H}_{2} \mathrm{O}\end{array}$} & 18.5 & & & & & (9) \\
\hline & 18.5 & $1455 / 1447$ & & $1.84 / 2.39$ & 231 & $(4,13,15)$ \\
\hline \multirow{9}{*}{$\begin{array}{l}\text { Calcium chloride hexahydrate } \\
\mathrm{CaCl}_{2} \cdot 6 \mathrm{H}_{2} \mathrm{O}\end{array}$} & 24 & $/ 1470$ & $1.09 / 0.54$ & $1.4 / 2.1$ & 140 & (87) \\
\hline & 28 & $1710 / 1500$ & $1.09 / 0.54$ & $1.43 / 2.31$ & 188.34 & $(88)$ \\
\hline & 28 & $1680 / 1500$ & $0.624 / 0.454$ & $1.25 / 2.13$ & 200 & $(1,89)$ \\
\hline & 29 & $1630 / 1500$ & & & 160 & (44) \\
\hline & 29.2 & & & & & (9) \\
\hline & 29.30 & $1710 / 1562$ & $1.088 / 0.540$ & & 171.19 & (48) \\
\hline & 29.5 & $1680 /$ & & $1.42 / 2.3$ & 170 & $(7,20)$ \\
\hline & 29.6 & $1802 / 1562$ & $1.088 / 0.540$ & $1.42 / 2.1$ & 190.8 & $(5,20,46,90)$ \\
\hline & 29.7 & $1710 / 1496$ & & $1.45 /$ & 171 & (4) \\
\hline \multirow{2}{*}{$\begin{array}{l}\text { Manganese nitrate hexahydrate } \\
\mathrm{Mn}\left(\mathrm{NO}_{3}\right)_{2} \cdot 6 \mathrm{H}_{2} \mathrm{O}\end{array}$} & 25.8 & $1795 / 1738$ & $2.34 /$ & & 125.9 & $(46,49)$ \\
\hline & 26 & & & & 140 & $(7,20)$ \\
\hline \multirow{4}{*}{$\begin{array}{l}\text { Lithium nitrate trihydrate } \\
\mathrm{LiNO}_{3} \cdot 3 \mathrm{H}_{2} \mathrm{O}\end{array}$} & 29.9 & & & & 296 & $(5,20)$ \\
\hline & 30 & & & & 125 & $(7,20)$ \\
\hline & 30 & & & & 296 & $(13,46,91)$ \\
\hline & 30 & 1460 & & & 256 & (49) \\
\hline \multirow{8}{*}{$\begin{array}{l}\text { Sodium sulphate decahydrate } \\
\text { (Glauber's salt) } \\
\mathrm{Na}_{2} \mathrm{SO}_{4} \cdot 10 \mathrm{H}_{2} \mathrm{O}\end{array}$} & $21-23$ & 1480 & & & 198 & (92) \\
\hline & $31-32$ & $1534 /$ & & & 251 & (44) \\
\hline & 32.4 & & & & 251 & (9) \\
\hline & 32.4 & $1460 / 1330$ & $0.7 / 0.544$ & $1.76 / 3.3$ & 241 & (93) \\
\hline & 32.4 & $1485 /$ & 0.544 & $1.93 /$ & 254 & $(4,46)$ \\
\hline & 32.4 & & & & 251 & $(7,20)$ \\
\hline & 32.5 & & & & 251 & (74) \\
\hline & 34 & 1550 & & & 176 & (49) \\
\hline \multirow{4}{*}{$\begin{array}{l}\text { Sodium carbonate decahydrate } \\
\mathrm{Na}_{2} \mathrm{CO}_{3} \cdot 10 \mathrm{H}_{2} \mathrm{O}\end{array}$} & $32-36$ & 1442 & & & 247 & (44) \\
\hline & 33 & & & & & (9) \\
\hline & 33 & $1460 /$ & & & 247 & $(5,20,91)$ \\
\hline & 34 & $1440 /$ & & $1.88 / 3.35$ & 251 & $(7,20)$ \\
\hline \multirow{3}{*}{$\begin{array}{l}\text { Calcium bromide hexahydrate } \\
\mathrm{CaBr}_{2} \cdot 6 \mathrm{H}_{2} \mathrm{O}\end{array}$} & 34 & $2194 / 1956$ & & & 115.5 & $(46,61)$ \\
\hline & 34 & & & & & (9) \\
\hline & 34.3 & $2194 / 1956$ & & & 115.5 & $(5,20,46)$ \\
\hline \multirow{3}{*}{$\begin{array}{l}\text { Disodium hydrophosphate } \\
\text { dodecahydrate } \\
\mathrm{Na}_{2} \mathrm{HPO}_{4} \cdot 12 \mathrm{H}_{2} \mathrm{O}\end{array}$} & 35 & $1520 / 1442$ & $0.514 / 0.476$ & $1.70 / 1.95$ & 281 & (4) \\
\hline & 35.2 & & & & & (9) \\
\hline & $35.2-44.6$ & $1520 / 1442$ & $0.514 / 0.476$ & $1.70 / 1.95$ & 280 & $(5,20)$ \\
\hline
\end{tabular}


TABLE 15. Continued

\begin{tabular}{|c|c|c|c|c|c|c|}
\hline Material & $\begin{array}{l}\text { Melting } \\
\text { temp. }\left({ }^{\circ} \mathbf{C}\right)\end{array}$ & $\begin{array}{l}\text { Density } \\
\left(\mathrm{kg} / \mathrm{m}^{3}\right)\end{array}$ & $\begin{array}{c}\text { Thermal conductivity } \\
(\mathrm{W} / \mathrm{m} \mathrm{K})\end{array}$ & $\begin{array}{l}\text { Specific heat } \\
(\mathbf{k J} / \mathrm{kg} \mathrm{K})\end{array}$ & $\begin{array}{l}\text { Latent heat } \\
(\mathrm{kJ} / \mathrm{kg})\end{array}$ & Reference \\
\hline \multirow{6}{*}{$\begin{array}{l}\text { Zinc nitrate hexahydrate } \\
\mathrm{Zn}\left(\mathrm{NO}_{3}\right)_{2} \cdot 6 \mathrm{H}_{2} \mathrm{O}\end{array}$} & 36.5 & 1520 & & $1.55 / 3.18$ & 264 & $(7,20)$ \\
\hline & $38-41.5$ & $1522 / 1442$ & & & 269.7 & (44) \\
\hline & 36 & & & & & (9) \\
\hline & 36 & $1937 / 1828$ & $/ 0.464$ & & 146.9 & $(5,46)$ \\
\hline & 36.4 & $2065 /$ & & $1.34 / 2.26$ & 147 & (4) \\
\hline & 36.4 & 2070 & & $1.34 / 2.26$ & 130 & $(7,20)$ \\
\hline $\begin{array}{l}\text { Potassium fluoride dihydrate } \\
\mathrm{KF} \cdot 2 \mathrm{H}_{2} \mathrm{O}\end{array}$ & 41.4 & & & & & (9) \\
\hline $\begin{array}{l}\text { Potassium acetate monohydrate } \\
\mathrm{K}\left(\mathrm{CH}_{3} \mathrm{COO}\right) \cdot 1 \frac{1}{2} \mathrm{H}_{2} \mathrm{O}\end{array}$ & 42 & & & & & $(46,86)$ \\
\hline \multirow{3}{*}{$\begin{array}{l}\text { Calcium nitrate tetrahydrate } \\
\mathrm{Ca}\left(\mathrm{NO}_{3}\right)_{2} \cdot 4 \mathrm{H}_{2} \mathrm{O}\end{array}$} & 42.6 & $1820 /$ & & $1.46 /$ & 140 & $(7,20)$ \\
\hline & 42.7 & & & & 142 & $(20,94)$ \\
\hline & 42.7 & & & & & (9) \\
\hline $\begin{array}{l}\text { Potassium phosphate } \\
\text { heptahydrate } \\
\mathrm{K}_{3} \mathrm{PO}_{4} \cdot 7 \mathrm{H}_{2} \mathrm{O}\end{array}$ & 45 & & & & & $(46,86)$ \\
\hline $\begin{array}{l}\text { Zinc nitrate tetrahydrate } \\
\mathrm{Zn}\left(\mathrm{NO}_{3}\right)_{2} \cdot 4 \mathrm{H}_{2} \mathrm{O}\end{array}$ & 45.5 & & & & & $(46,86)$ \\
\hline $\begin{array}{l}\text { Sodium phosphate heptahydrate } \\
\mathrm{Na}_{2} \mathrm{HPO}_{4} \cdot 7 \mathrm{H}_{2} \mathrm{O}\end{array}$ & 48 & & & & & (9) \\
\hline \multirow{7}{*}{$\begin{array}{l}\text { Sodium thiosulfate pentahydrate } \\
\mathrm{Na}_{2} \mathrm{~S}_{2} \mathrm{O}_{3} \cdot 5 \mathrm{H}_{2} \mathrm{O}\end{array}$} & 48.0 & $1730 / 1670$ & & $1.46 / 2.39$ & 201 & (4) \\
\hline & 48.0 & & & & 200 & $(20,94)$ \\
\hline & 48 & & & $/ 3.83$ & 206 & $(95)$ \\
\hline & 48 & & & & & (9) \\
\hline & $48.5-55.2$ & $1750 / 1670$ & & & 201 & $(5,20)$ \\
\hline & 49.0 & $1690 / 1660$ & & $1.46 / 2.38$ & & $(7,20)$ \\
\hline & $49.8-51.3$ & $1730 / 1660$ & & & 217.2 & (44) \\
\hline $\begin{array}{l}\text { Zinc nitrate dihydrate } \\
\mathrm{Zn}\left(\mathrm{NO}_{3}\right)_{2} \cdot 2 \mathrm{H}_{2} \mathrm{O}\end{array}$ & 54 & & & & & $(46,86)$ \\
\hline \multirow{9}{*}{$\begin{array}{l}\text { Sodium acetate trihydrate } \\
\mathrm{NaCH}_{3} \mathrm{COO} \cdot 3 \mathrm{H}_{2} \mathrm{O}\end{array}$} & $55.6-56.5$ & & & & $237-243$ & $(1,96)$ \\
\hline & 58.0 & $1450 /$ & & $1.97 / 3.35$ & 180 & $(7,20)$ \\
\hline & 58.0 & & & & 289 & $(20,94)$ \\
\hline & 58.0 & & & $2.26 / 3.33$ & 248 & (95) \\
\hline & 58 & $1450 /$ & & & 226 & $(46,91)$ \\
\hline & 58 & $1450 /$ & 0.6 & & 240 & $(97,98)$ \\
\hline & 58 & $1450 / 1280$ & & & 226.264 & $(48)$ \\
\hline & 58.4 & & & & & (9) \\
\hline & 58.5 & & & & 260 & (74) \\
\hline \multirow{3}{*}{$\begin{array}{l}\text { Sodium hydroxide monohydrate } \\
\mathrm{NaOH} \cdot \mathrm{H}_{2} \mathrm{O}\end{array}$} & 58.0 & & & $2.18 /$ & - & (9) \\
\hline & 64 & & & & 259 & (74) \\
\hline & 64 & & & & 272 & $(7,20)$ \\
\hline \multirow{2}{*}{$\begin{array}{l}\text { Cadmium nitrate tetrahydrate } \\
\mathrm{Cd}\left(\mathrm{NO}_{3}\right)_{2} \cdot 4 \mathrm{H}_{2} \mathrm{O}\end{array}$} & 59.5 & & & & & (9) \\
\hline & 59.5 & $2450 /$ & & $1.09 /$ & 106 & $(7,20)$ \\
\hline $\begin{array}{l}\text { Iron nitrate hexahydrate } \\
\mathrm{Fe}\left(\mathrm{NO}_{3}\right)_{2} \cdot 6 \mathrm{H}_{2} \mathrm{O}\end{array}$ & 60 & & & & & $(46,86)$ \\
\hline $\begin{array}{l}\text { Sodium tetraborane decahydrate } \\
\mathrm{Na}_{2} \mathrm{~B}_{4} \mathrm{O}_{7} \cdot 10 \mathrm{H}_{2} \mathrm{O}\end{array}$ & 68.1 & & & & & (9) \\
\hline $\begin{array}{l}\text { Sodium phosphate dodecahydrate } \\
\mathrm{Na}_{3} \mathrm{PO}_{4} \cdot 12 \mathrm{H}_{2} \mathrm{O}\end{array}$ & 69.0 & & & & & (9) \\
\hline
\end{tabular}


TABLE 15. Continued

\begin{tabular}{|c|c|c|c|c|c|c|}
\hline Material & $\begin{array}{l}\text { Melting } \\
\text { temp. }\left({ }^{\circ} \mathbf{C}\right)\end{array}$ & $\begin{array}{l}\text { Density } \\
\left(\mathrm{kg} / \mathrm{m}^{3}\right)\end{array}$ & $\begin{array}{c}\text { Thermal conductivity } \\
(\mathrm{W} / \mathrm{m} \mathrm{K})\end{array}$ & $\begin{array}{c}\text { Specific heat } \\
(\mathrm{kJ} / \mathrm{kg} \mathrm{K})\end{array}$ & $\begin{array}{l}\text { Latent heat } \\
(\mathrm{kJ} / \mathrm{kg})\end{array}$ & Reference \\
\hline $\begin{array}{l}\text { Sodium pyrophosphate } \\
\text { decahydrate } \\
\mathrm{Na}_{4} \mathrm{P}_{2} \mathrm{O}_{7} \cdot 10 \mathrm{H}_{2} \mathrm{O}\end{array}$ & 70 & & & & 184 & $(46,91)$ \\
\hline $\begin{array}{l}\text { Aluminium nitrate nonahydrate } \\
\mathrm{AI}\left(\mathrm{NO}_{3}\right)_{2} \cdot 9 \mathrm{H}_{2} \mathrm{O}\end{array}$ & 70 & & & & & (9) \\
\hline \multirow{7}{*}{$\begin{array}{l}\text { Barium hydroxide octahydrate } \\
\mathrm{Ba}(\mathrm{OH})_{2} \cdot 8 \mathrm{H}_{2} \mathrm{O}\end{array}$} & 78.0 & $2180 /$ & & $1.17 /$ & 267 & (4) \\
\hline & 78.0 & $2180 /$ & & $1.17 /$ & 301 & $(7,20)$ \\
\hline & 78.0 & $2070 / 1937$ & $1.225 / 0.653$ & & 265.7 & $(5,20,90)$ \\
\hline & 78.0 & & & & 295 & $(20,94)$ \\
\hline & 78 & $2070 /$ & & & 280 & $(46,91)$ \\
\hline & 78 & & & & & (9) \\
\hline & 78 & & & & 301 & (74) \\
\hline \multirow{2}{*}{$\begin{array}{l}\text { Potassium alum dodecahydrate } \\
\mathrm{KAl}\left(\mathrm{SO}_{4}\right)_{2} \cdot 12 \mathrm{H}_{2} \mathrm{O}\end{array}$} & 80 & & & & & $(46,86)$ \\
\hline & 85.8 & & & & & (9) \\
\hline $\begin{array}{l}\text { Aluminium sulphate } \\
\text { octadecahydrate } \\
\mathrm{Al}_{2}\left(\mathrm{SO}_{4}\right)_{3} \cdot 18 \mathrm{H}_{2} \mathrm{O}\end{array}$ & 88 & & & & & (9) \\
\hline $\begin{array}{l}\text { Aluminium nitrate octahydrate } \\
\mathrm{Al}\left(\mathrm{NO}_{3}\right)_{3} \cdot 8 \mathrm{H}_{2} \mathrm{O}\end{array}$ & 89 & & & & & $(46,86)$ \\
\hline \multirow{3}{*}{$\begin{array}{l}\text { Magnesium nitrate hexahydrate } \\
\mathrm{Mg}\left(\mathrm{NO}_{3}\right)_{2} \cdot 6 \mathrm{H}_{2} \mathrm{O}\end{array}$} & 89 & $1636 / 1550$ & $0.669 / 0.490$ & $1810 / 2480$ & 162.8 & $(5,20,46)$ \\
\hline & 90.0 & $1460 /$ & & $2260 / 3680$ & 160 & $(7,20)$ \\
\hline & 90 & & & & 150.3 & (9) \\
\hline $\begin{array}{l}\text { Ammonium aluminium sulphate } \\
\text { hexahydrate } \\
\left(\mathrm{NH}_{4}\right) \mathrm{Al}\left(\mathrm{SO}_{4}\right) \cdot 6 \mathrm{H}_{2} \mathrm{O}\end{array}$ & 95 & & & & 269 & $(46,86,91)$ \\
\hline \multirow{2}{*}{$\begin{array}{l}\text { Ammonium aluminium sulphate } \\
\text { dodecahydrate } \\
\left(\mathrm{NH}_{4}\right) \mathrm{Al}\left(\mathrm{SO}_{4}\right)_{2} \cdot 12 \mathrm{H}_{2} \mathrm{O}\end{array}$} & 94.0 & $1650 /$ & & $1.71 / 3.05$ & 269 & $(5,20)$ \\
\hline & 94 & & & & & (9) \\
\hline $\begin{array}{l}\text { Sodium sulphide pentahydrate } \\
\mathrm{Na}_{2} \mathrm{~S} \cdot 5 \mathrm{H}_{2} \mathrm{O}\end{array}$ & 96 & & & & 320.8 & (9) \\
\hline $\begin{array}{l}\text { Sodium sulphide pentahydrate } \\
\mathrm{Na}_{2} \mathrm{~S} \cdot 5 \frac{1}{2} \mathrm{H}_{2} \mathrm{O}\end{array}$ & 97.5 & & & & & $(46,86)$ \\
\hline $\begin{array}{l}\text { Bischofite } \\
\text { (main component } \mathrm{MgCl}_{2} \cdot 6 \mathrm{H}_{2} \mathrm{O} \text { ) }\end{array}$ & 98.8 & $1686 / 1481$ & & $1.6 / 3.0$ & 120.2 & (99) \\
\hline $\begin{array}{l}\text { Calcium bromide tetrahydrate } \\
\mathrm{CaBr}_{2} \cdot 4 \mathrm{H}_{2} \mathrm{O}\end{array}$ & 110 & & & & & $(46,86)$ \\
\hline $\begin{array}{l}\text { Aluminium sulfate } \\
\text { hexadecahydrate } \\
\mathrm{Al}_{2}\left(\mathrm{SO}_{4}\right)_{3} \cdot 16 \mathrm{H}_{2} \mathrm{O}\end{array}$ & 112 & & & & & $(46,86)$ \\
\hline \multirow{5}{*}{$\begin{array}{l}\text { Magnesium chloride hexahydrate } \\
\mathrm{MgCl}_{2} \cdot 6 \mathrm{H}_{2} \mathrm{O}\end{array}$} & 114.6 & $1517 / 1422$ & & $2.1 / 1.95$ & 126.6 & (99) \\
\hline & 116 & $1570 / 1442$ & & $1.72 / 2.82$ & 165 & (4) \\
\hline & 117.0 & $1560 /$ & & $1.59 / 2.85$ & 172 & $(7,20)$ \\
\hline & 117 & $1569 / 1450$ & $0.694 / 0.570$ & $2.25 / 2.61$ & 168.6 & $(5,20,46)$ \\
\hline & 117 & & & & 259.0 & (9) \\
\hline
\end{tabular}

4.2.3.1. Organic-organic eutectics Different organicorganic eutectics and compositions are found in literature and listed in Table 17. Main tested organic eutectics are based on fatty acid mixtures, such as capric acid + lauric acid (CA + LA), capric acid + palmitic acid $(\mathrm{CA}+\mathrm{PA})$, lauric acid-palmitic acid
(LA-PA), lauric acid-myristic acid (LA-MA) or myristic acid-stearic acid (MA-SA) $(50,64,103,104)$. Thermo-physical properties of theses mixtures have been tested at different weight combinations by DSC analysis with the aim of identifying the eutectic mixture concentration. It has been shown that the melting 


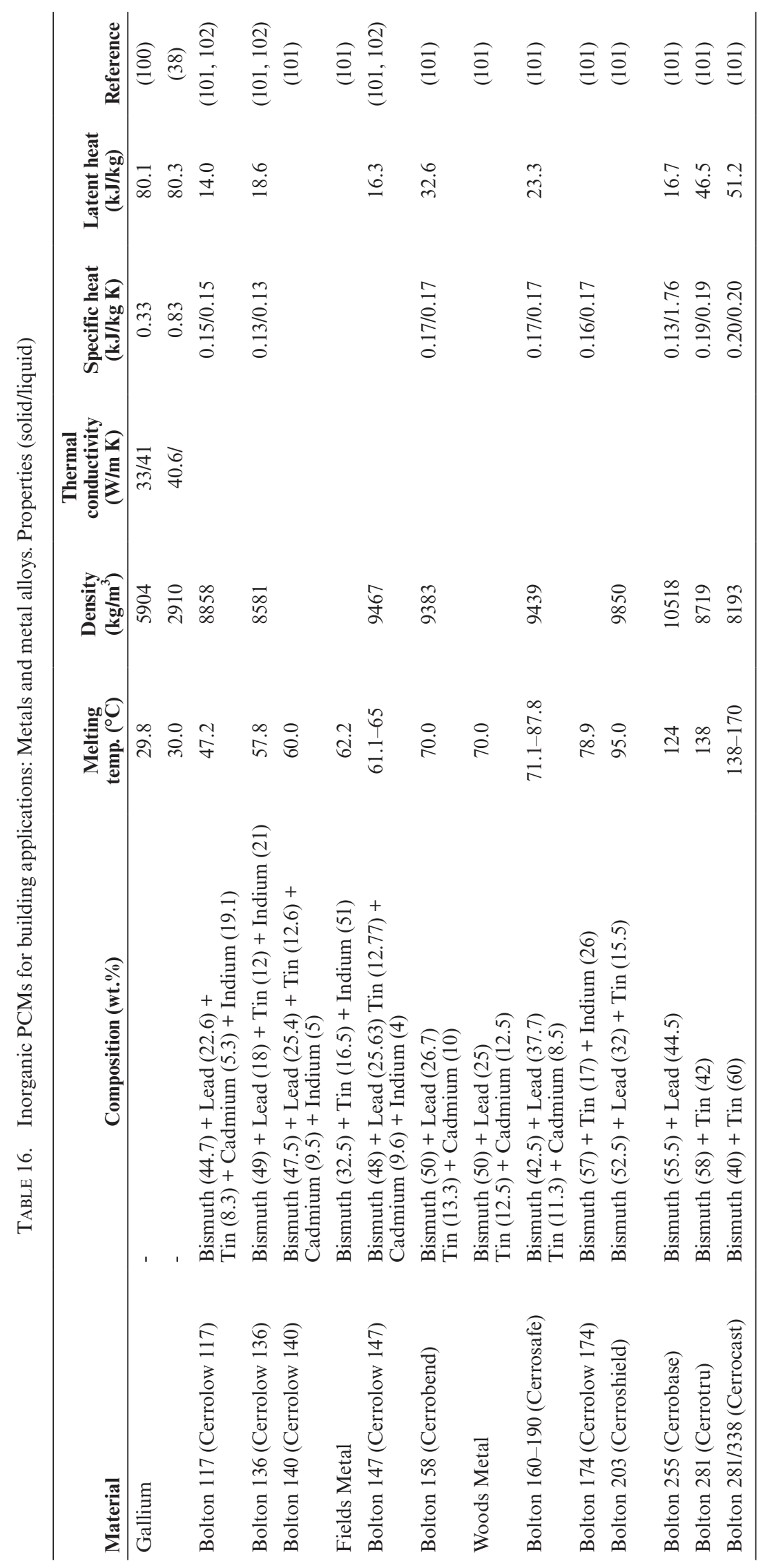


TABLE 17. Eutectics and compositions for latent heat storage. Organic-Organic. Properties (solid/liquid)

\begin{tabular}{|c|c|c|c|c|c|c|c|}
\hline Material & $\begin{array}{l}\text { Composition } \\
\text { (wt.\%) }\end{array}$ & $\begin{array}{l}\text { Melting } \\
\text { temp. }\left({ }^{\circ} \mathbf{C}\right)\end{array}$ & $\begin{array}{l}\text { Density } \\
\left(\mathrm{kg} / \mathrm{m}^{3}\right)\end{array}$ & $\begin{array}{c}\text { Thermal } \\
\text { conductivity } \\
(\mathrm{W} / \mathrm{m} \text { K) }\end{array}$ & $\begin{array}{c}\text { Specific } \\
\text { heat } \\
\text { (kJ/kg K) }\end{array}$ & $\begin{array}{c}\text { Latent } \\
\text { heat }(\mathrm{kJ} / \\
\mathrm{kg})\end{array}$ & References \\
\hline \multirow[t]{3}{*}{ Capric-lauric acid + Pentadecane } & $50+50$ & 10.2 & $850.4 / 827.8$ & & $2.44 / 2.89$ & 157.8 & $(50)$ \\
\hline & $70+30$ & 11.3 & $872.7 / 858.0$ & & $2.27 / 2.57$ & 149.2 & $(50)$ \\
\hline & $90+10$ & 13.3 & $891.3 / 883.2$ & & $2.08 / 2.42$ & 142.2 & $(46,50)$ \\
\hline Triethylolethane $+\mathrm{H}_{2} \mathrm{O}+\mathrm{CO}\left(\mathrm{NH}_{2}\right)_{2}$ & $38.5+31.5+30$ & 13.4 & - & - & & 160 & $(10)$ \\
\hline \multirow[t]{4}{*}{ Capric acid + lauric acid } & $45+55$ & $17-21$ & & & & 143 & $(75)$ \\
\hline & $65+35$ & 18 & $900.0 / 894.9$ & $0.143 / 0.139$ & $1.97 / 2.24$ & 140.8 & $(50,103)$ \\
\hline & & 18 & & & & 120 & (44) \\
\hline & $45+55$ & 21 & - & - & - & 143 & $(46,76)$ \\
\hline \multirow[t]{2}{*}{ Capric acid + palmitic acid } & $76.5+23.5$ & 21.8 & & & & 171.2 & $(1,64)$ \\
\hline & $75.2+24.8$ & 22.5 & $870 / 790$ & $0.14 / 0.14$ & $2 / 2.3$ & 173 & $(29)$ \\
\hline Mystiric acid + Capric acid & $34+66$ & 24 & $1018 / 888$ & $/ 0.164$ & - & 147.7 & $(5,46)$ \\
\hline Mystiric acid + Decanoic acid & $34+66$ & 24 & - & - & & 147.7 & $(10)$ \\
\hline Capric acid + stearic acid & $86+14$ & 26.04 & & & & 176.68 & $(22,108)$ \\
\hline $\begin{array}{l}\text { Acetamide + Urea } \\
\mathrm{CH}_{3} \mathrm{CONH}_{2}+\mathrm{CO}\left(\mathrm{NH}_{2}\right)_{2}\end{array}$ & $50+50$ & 27 & - & - & & 163 & (10) \\
\hline Triethylolethane $+\mathrm{CO}\left(\mathrm{NH}_{2}\right)_{2}$ & $62.5+37.5$ & 29.8 & - & - & & 218 & $(10)$ \\
\hline \multirow[t]{2}{*}{ Lauric acid + Palmitic acid } & & 33 & & & & 145 & (44) \\
\hline & $69.0+31.0$ & 35.2 & & & & 166.3 & $(1,104)$ \\
\hline \multirow[t]{2}{*}{ Lauric acid + Stearic acid } & & 34 & & & & 150 & $(44)$ \\
\hline & $75.5+24.5$ & 37 & & & & 182.7 & $(1,107)$ \\
\hline Lauric acid + Myristic acid & $66.0+34.0$ & 34.2 & & & & 166.8 & $(1,67,104)$ \\
\hline Myristic acid + Palmitic acid & $58.0+42.0$ & 42.6 & & & & 169.7 & $(1,106)$ \\
\hline \multirow[t]{2}{*}{ Myristic acid + Stearic acid } & $64.0+36.0$ & 44.1 & & & & 182.4 & $(1,104,109)$ \\
\hline & $65.7+34.3$ & 50.2 & & & & 162.0 & $(1,110)$ \\
\hline Propionamide + Palmitic acid & $25.1+74.9$ & 50 & & & $1.96 / 2.40$ & 192 & (4) \\
\hline \multirow[t]{2}{*}{ Palmitic acid + Stearic acid } & & 51 & & & & 160 & (44) \\
\hline & $64.2+35.8$ & 52.3 & & & & 181.7 & $(1,105)$ \\
\hline $\begin{array}{l}\text { Acetamide }+ \text { Stearic acid } \\
\mathrm{CH}_{3} \mathrm{CONH}_{2}+\mathrm{C}_{17} \mathrm{H}_{35} \mathrm{COOH}\end{array}$ & $50+50$ & 65 & - & - & & 218 & $(10)$ \\
\hline $\begin{array}{l}\text { Naphthalene }+ \text { benzoic acid } \\
\mathrm{C}_{10} \mathrm{H}_{8}+\mathrm{C}_{6} \mathrm{H}_{5} \mathrm{COOH}\end{array}$ & $67.1+32.9$ & 67 & & $0.282 / 0.136$ & & 123.4 & $(5,46)$ \\
\hline $\begin{array}{l}\text { Naphthalene + benzoic acid } \\
\mathrm{C}_{10} \mathrm{H}_{8}+\mathrm{C}_{6} \mathrm{H}_{5} \mathrm{COOH}\end{array}$ & $67.1+32.9$ & 67 & - & - & & 123.4 & $(10)$ \\
\hline
\end{tabular}

temperature and the latent heat in the mixture of these materials follow a downtrend process through different concentrations until the eutectic proportion is achieved $(64,67,105-107)$.

4.2.3.2. Organic-inorganic eutectics Organicinorganic eutectics and compositions tested are listed in Table 18. They are mainly based on the mixture of urea and salt.

4.2.3.3. Inorganic-inorganic eutectics Different inorganic-inorganic eutectics and compositions are listed in Table 19. Main inorganic eutectics are based on alloys of salt hydrates.

\subsection{Thermochemical storage materials}

Thermochemical energy storage is a promising technology mainly due to the high energy density potentially achievable and the possibility of storing energy for long-term periods with negligible heat losses. Nevertheless, research in this field is yet at an early stage (38) and TSMs are not currently available as commercial solutions for TES in buildings. The higher complexity of these storage systems in comparison with currently used sensible heat materials, the higher cost of some of these compounds as well as the need to increase heat and mass transfer capacity to release the stored energy are main challenges 
TABLE 18. Eutectics and compositions for latent heat storage. Organic-Inorganic. Properties (solid/liquid)

\begin{tabular}{|c|c|c|c|c|c|c|c|}
\hline Material & $\begin{array}{l}\text { Composition } \\
\text { (wt.\%) }\end{array}$ & $\begin{array}{l}\text { Melting } \\
\text { temp. }\left({ }^{\circ} \mathbf{C}\right)\end{array}$ & $\begin{array}{l}\text { Density } \\
\left(\mathrm{kg} / \mathrm{m}^{3}\right)\end{array}$ & $\begin{array}{l}\text { Thermal } \\
\text { conductivity } \\
(\mathrm{W} / \mathrm{m} \mathrm{K})\end{array}$ & $\begin{array}{l}\text { Specific heat } \\
(\mathrm{kJ} / \mathrm{kg} \mathrm{K})\end{array}$ & $\begin{array}{l}\text { Latent heat } \\
(\mathbf{k J} / \mathbf{k g})\end{array}$ & Reference \\
\hline \multirow{3}{*}{$\begin{array}{l}\text { Sodium acetate }+ \text { Urea } \\
\mathrm{Na}\left(\mathrm{CH}_{3} \mathrm{COO}\right) \cdot 3 \mathrm{H}_{2} \mathrm{O}+\mathrm{CO}\left(\mathrm{NH}_{2}\right)_{2}\end{array}$} & $60+40$ & 30 & & & & 200.5 & $(46,111)$ \\
\hline & $40+60$ & 30 & & & & 200.5 & $(1,10)$ \\
\hline & $60+40$ & 31.5 & & & & 226 & (74) \\
\hline $\begin{array}{l}\text { Urea }+ \text { Ammonium nitrate } \\
\mathrm{CO}\left(\mathrm{NH}_{2}\right)_{2}+\mathrm{NH}_{4} \mathrm{NO}_{3}\end{array}$ & $53+47$ & 46 & - & - & & 95 & (10) \\
\hline \multirow{2}{*}{$\begin{array}{l}\text { Urea }+ \text { Ammonium bromide } \\
\mathrm{CO}\left(\mathrm{NH}_{2}\right)_{2}+\mathrm{NH}_{4} \mathrm{Br}\end{array}$} & $66.6+33.4$ & 76 & $1548 / 1440$ & $0.682 / 0.331$ & & 161.0 & $(1,5,46)$ \\
\hline & $66.6+33.4$ & 76 & - & - & & 151 & (10) \\
\hline
\end{tabular}

TABLE 19. Eutectics and compositions for latent heat storage. Inorganic-Inorganic. Properties (solid/liquid)

\begin{tabular}{|c|c|c|c|c|c|c|c|}
\hline Material & Composition (wt.\%) & $\begin{array}{l}\text { Melting } \\
\text { temp. }\left({ }^{\circ} \mathbf{C}\right) \\
\end{array}$ & $\begin{array}{l}\text { Density } \\
\left(\mathrm{kg} / \mathrm{m}^{3}\right)\end{array}$ & $\begin{array}{c}\text { Thermal } \\
\text { conductivity } \\
(\mathrm{W} / \mathrm{m} \mathrm{K})\end{array}$ & $\begin{array}{c}\text { Specific } \\
\text { heat } \\
(\mathbf{k J} / \mathbf{k g ~ K}) \\
\end{array}$ & $\begin{array}{c}\text { Latent } \\
\text { heat } \\
(\mathrm{kJJ} / \mathrm{kg})\end{array}$ & Reference \\
\hline $\mathrm{NaNO}_{3}+\mathrm{H}_{2} \mathrm{O}$ & $36.9+63.1$ & -18.5 & & & & 212 & (74) \\
\hline$\left(\mathrm{NH}_{4}\right)_{2} \mathrm{SO}_{4}+\mathrm{H}_{2} \mathrm{O}$ & $39.7+60.3$ & -18.5 & & & & 1.59 & (74) \\
\hline $\mathrm{NH}_{4} \mathrm{Cl}+\mathrm{H}_{2} \mathrm{O}$ & $19.5+80.5$ & -16 & $1020 / 1060$ & & 13.2 & 286 & (74) \\
\hline $\mathrm{KCl}+\mathrm{H}_{2} \mathrm{O}$ & $19.5+80.5$ & -10.7 & $1050 / 1130$ & & 13.24 & 283 & (74) \\
\hline $\mathrm{ZnSO}_{4}+\mathrm{H}_{2} \mathrm{O}$ & $27.2+72.8$ & -6.5 & $/ 1340$ & & & 150 & (74) \\
\hline $\mathrm{NH}_{4} \mathrm{H}_{2} \mathrm{PO}_{4}+\mathrm{H}_{2} \mathrm{O}$ & $16.5+83.5$ & -4.0 & $1040 / 1090$ & & /3.64 & 164 & (74) \\
\hline $\mathrm{MgSO}_{4}+\mathrm{H}_{2} \mathrm{O}$ & $19+81$ & -3.9 & $990 / 1040$ & & /3.81 & 286 & (74) \\
\hline $\mathrm{NaF}+\mathrm{H}_{2} \mathrm{O}$ & $3.9+96.1$ & -3.5 & $960 / 1040$ & & 13.85 & 323 & (74) \\
\hline $\mathrm{Ca}\left(\mathrm{NO}_{3}\right)_{2}+\mathrm{H}_{2} \mathrm{O}$ & $9.7+90.3$ & -2.8 & 990/ & & /3.61 & 281 & (74) \\
\hline $\mathrm{Na}_{2} \mathrm{SO}_{4}+\mathrm{NaCl}+\mathrm{KCl}+\mathrm{H}_{2} \mathrm{O}$ & $31+13+16+40$ & 4 & & & - & 234 & (4) \\
\hline $\mathrm{Na}_{2} \mathrm{SO}_{4}+\mathrm{H}_{2} \mathrm{O}+\mathrm{NaCl}+\mathrm{NH}_{4} \mathrm{Cl}$ & $32.5+41.4+6.66+6.16$ & 13 & & & & 146 & (44) \\
\hline \multirow[t]{2}{*}{$\mathrm{CaBr}_{2} \cdot 6 \mathrm{H}_{2} \mathrm{O}+\mathrm{CaCl}_{2} \cdot 6 \mathrm{H}_{2} \mathrm{O}$} & $55+45$ & 14 & & & & 140 & $(44)$ \\
\hline & $55+45$ & 14.7 & & & & 140 & $(10,74)$ \\
\hline $\mathrm{Cu}\left(\mathrm{NO}_{3}\right)_{3} \cdot 6 \mathrm{H}_{2} \mathrm{O}+\mathrm{LiNO}_{3} \cdot 3 \mathrm{H}_{2} \mathrm{O}$ & $55+45$ & 16.5 & & & & 250 & (74) \\
\hline $\mathrm{LiNO}_{3} \cdot 3 \mathrm{H}_{2} \mathrm{O}+\mathrm{Zn}\left(\mathrm{NO}_{3}\right)_{2} \cdot 6 \mathrm{H}_{2} \mathrm{O}$ & $45+55$ & 17.2 & & & & 220 & (74) \\
\hline $\mathrm{Na}_{2} \mathrm{SO}_{4} \cdot 10 \mathrm{H}_{2} \mathrm{O}+\mathrm{NaCl}$ & $50+50$ & 18 & & & & & (49) \\
\hline $\begin{array}{l}\mathrm{Co}\left(\mathrm{NO}_{3}\right)_{2} \cdot 6 \mathrm{H}_{2} \mathrm{O}+\mathrm{H}_{2} \mathrm{O}+ \\
\mathrm{LiNO}_{3} \cdot 3 \mathrm{H}_{2} \mathrm{O}\end{array}$ & $47+7.75+45.25$ & 22.3 & & & & 265 & (74) \\
\hline $\mathrm{LiNO}_{3} \cdot 3 \mathrm{H}_{2} \mathrm{O}+\mathrm{Ni}\left(\mathrm{NO}_{3}\right)_{2} \cdot 6 \mathrm{H}_{2} \mathrm{O}$ & $60+40$ & 24.2 & & & & 230 & (74) \\
\hline $\mathrm{Ca}\left(\mathrm{NO}_{3}\right)_{2} \cdot 6 \mathrm{H}_{2} \mathrm{O}+\mathrm{Zn}\left(\mathrm{NO}_{3}\right)_{2} \cdot 6 \mathrm{H}_{2} \mathrm{O}$ & $45+55$ & 25 & $1930 /$ & & & 130 & $(74)$ \\
\hline $\mathrm{CaCl}_{2} \cdot 6 \mathrm{H}_{2} \mathrm{O}+\mathrm{MgCl}_{2} \cdot 6 \mathrm{H}_{2} \mathrm{O}$ & $66.6+33.3$ & 25 & & & & 127 & $(46,91)$ \\
\hline \multirow[t]{2}{*}{$\mathrm{CaCl}_{2}+\mathrm{NaCl}+\mathrm{KCl}+\mathrm{H}_{2} \mathrm{O}$} & $48+4.3+0.4+47.3$ & 26.8 & & & & & (4) \\
\hline & $48+4.3+0.4+47.3$ & 26.8 & 1640 & & & 188.0 & $(46,91)$ \\
\hline $\mathrm{Cd}\left(\mathrm{NO}_{3}\right)_{2} \cdot 6 \mathrm{H}_{2} \mathrm{O}+\mathrm{Zn}\left(\mathrm{NO}_{3}\right)_{2} \cdot 6 \mathrm{H}_{2} \mathrm{O}$ & $60+40$ & 27.4 & & & & 126 & $(74)$ \\
\hline $\mathrm{Ca}\left(\mathrm{NO}_{3}\right)_{2} 4 \mathrm{H}_{2} \mathrm{O}+\mathrm{Zn}\left(\mathrm{NO}_{3}\right)_{2} \cdot 6 \mathrm{H}_{2} \mathrm{O}$ & $50+50$ & 29.2 & & & & 130 & (74) \\
\hline \multirow[t]{3}{*}{$\mathrm{Ca}\left(\mathrm{NO}_{3}\right)_{2} \cdot 4 \mathrm{H}_{2} \mathrm{O}+\mathrm{Mg}\left(\mathrm{NO}_{3}\right)_{2} \cdot 6 \mathrm{H}_{2} \mathrm{O}$} & $67+33$ & 30 & & & & 136 & (4) \\
\hline & $67+33$ & 30 & $1670 /$ & & & 135 & $(74)$ \\
\hline & $47+53$ & 30 & & & & 136 & $(1,10)$ \\
\hline $\mathrm{Zn}\left(\mathrm{NO}_{3}\right)_{2} \cdot 6 \mathrm{H}_{2} \mathrm{O}+\mathrm{Zn}\left(\mathrm{NO}_{3}\right)_{2} \cdot 6 \mathrm{H}_{2} \mathrm{O}$ & $82+18$ & 32 & 1910/ & & & 136 & $(74)$ \\
\hline $\mathrm{Ca}\left(\mathrm{NO}_{3}\right)_{2} \cdot 4 \mathrm{H}_{2} \mathrm{O}+\mathrm{Al}\left(\mathrm{NO}_{3}\right)_{3} \cdot 9 \mathrm{H}_{2} \mathrm{O}$ & $72+28$ & 35 & $1720 /$ & & & 139 & (74) \\
\hline $\mathrm{Ni}\left(\mathrm{NO}_{3}\right)_{2} \cdot 6 \mathrm{H}_{2} \mathrm{O}+\mathrm{Cd}\left(\mathrm{NO}_{3}\right)_{2} \cdot 6 \mathrm{H}_{2} \mathrm{O}$ & $50+50$ & 37.5 & & & & - & (74) \\
\hline $\mathrm{MgCl} \cdot 6 \mathrm{H}_{2} \mathrm{O}+\mathrm{Mg}\left(\mathrm{NO}_{3}\right)_{2} \cdot 6 \mathrm{H}_{2} \mathrm{O}$ & $49.3+50.7$ & 58 & & & & 132.3 & $(44)$ \\
\hline
\end{tabular}


Table 19. Continued

\begin{tabular}{|c|c|c|c|c|c|c|c|}
\hline Material & Composition (wt.\%) & $\begin{array}{l}\text { Melting } \\
\text { temp. }\left({ }^{\circ} \mathbf{C}\right)\end{array}$ & $\begin{array}{l}\text { Density } \\
\left(\mathrm{kg} / \mathrm{m}^{3}\right)\end{array}$ & $\begin{array}{l}\text { Thermal } \\
\text { conductivity } \\
\text { (W/m K) }\end{array}$ & $\begin{array}{c}\text { Specific } \\
\text { heat } \\
(\mathrm{kJ} / \mathrm{kg} \mathrm{K})\end{array}$ & $\begin{array}{c}\text { Latent } \\
\text { heat } \\
(\mathrm{kJ} / \mathrm{kg})\end{array}$ & Reference \\
\hline \multirow[t]{3}{*}{$\mathrm{Mg}\left(\mathrm{NO}_{3}\right) \cdot 6 \mathrm{H}_{2} \mathrm{O}+\mathrm{NH}_{4} \mathrm{NO}_{3}$} & $61.5+38.5$ & 51 & & & & 131.3 & (44) \\
\hline & $53+47$ & 52 & & & & 125 & $(74)$ \\
\hline & $61.5+38.5$ & 52 & $1596 / 1515$ & $0.552 / 0.494$ & & 125.5 & $(5,46)$ \\
\hline \multirow[t]{2}{*}{$\mathrm{Mg}\left(\mathrm{NO}_{3}\right) \cdot 6 \mathrm{H}_{2} \mathrm{O}+\mathrm{MgCl}_{2} \cdot 6 \mathrm{H}_{2} \mathrm{O}$} & $58.3+41.7$ & 58 & & & & 106 & $(44)$ \\
\hline & & 58 & & & & 132 & $(46,91)$ \\
\hline \multirow[t]{4}{*}{$\mathrm{Mg}\left(\mathrm{NO}_{3}\right)_{2} \cdot 6 \mathrm{H}_{2} \mathrm{O}+\mathrm{MgCl}_{2} \cdot 6 \mathrm{H}_{2} \mathrm{O}$} & $58.7+41.3$ & 59 & $1630 / 1550$ & $0.678 / 0.51$ & & 132.2 & $(5,46)$ \\
\hline & $53+47$ & 59.1 & & & $1.34 / 3.16$ & 144 & (4) \\
\hline & $61.5+38.5$ & 59.1 & $1680 /$ & & & 144 & (74) \\
\hline & $58.7+41.3$ & 59.1 & & & & 132.2 & (9) \\
\hline \multirow[t]{2}{*}{$\mathrm{Mg}\left(\mathrm{NO}_{3}\right)_{2} \cdot 6 \mathrm{H}_{2} \mathrm{O}+\mathrm{Al}\left(\mathrm{NO}_{3}\right)_{2} \cdot 9 \mathrm{H}_{2} \mathrm{O}$} & $53+47$ & 61 & & & & 148 & (4) \\
\hline & $53+47$ & 61 & $1850 /$ & & & 148 & (74) \\
\hline $\mathrm{Mg}\left(\mathrm{NO}_{3}\right)_{2} \cdot 6 \mathrm{H}_{2} \mathrm{O}+\mathrm{MgBr}_{2} \cdot 6 \mathrm{H}_{2} \mathrm{O}$ & $59+41$ & 66 & & & & 168 & (10) \\
\hline $\mathrm{LiNO}_{3}+\mathrm{Mg}\left(\mathrm{NO}_{3}\right)_{2} \cdot 6 \mathrm{H}_{2} \mathrm{O}$ & $14+86$ & 72 & $1610 / 1590$ & & & 180 & $(1,46,91)$ \\
\hline $\mathrm{LiNO}_{3}+\mathrm{NH}_{4} \mathrm{NO}_{3}+\mathrm{NaNO}_{3}$ & $25+65+10$ & 80.5 & & & & 113 & (10) \\
\hline $\mathrm{LiNO}_{3}+\mathrm{NH}_{4} \mathrm{NO}_{3}+\mathrm{KNO}_{3}$ & $26.4+58.7+14.9$ & 81.5 & & & & 116 & $(10)$ \\
\hline \multirow[t]{2}{*}{$\mathrm{LiNO}_{3}+\mathrm{NH}_{4} \mathrm{NO}_{3}+\mathrm{NH}_{4} \mathrm{Cl}$} & $27+68+5$ & 81.6 & & & $1.07 / 2.20$ & 111 & $(4,74)$ \\
\hline & $27+68+5$ & 81.6 & & & & 108 & $(10)$ \\
\hline
\end{tabular}

for their commercial deployment (16). An evidence of the interest aroused by TCES is the number of international programmes and projects focused on the study of the most promising TSMs, such as the European projects HYDES, MODESTORE (2003-2012), MERITS (2007-20013), COMTES (2012-2016) or E-HUB (2010-2014), or the projects related to Task 32 (2003-2007) $(112,113)$, and Task 42 (2009-2015) (98) in the framework of the Solar Heating and Cooling Programme of the International Energy Agency (SHC-ECES).

Tested TSMs in the literature are classified into solid adsorption materials (zeolite and silica gel), liquid absorption materials, and chemical reactions through solid chemical reaction materials or composite materials (CSMP) $(16,17)$. This section reviews all different TSMs studied by different researchers for their potential use in low-to-moderate temperature applications.

\subsubsection{Sorption materials}

4.3.1.1. Physical adsorption materials Adsorption is based on the binding of a gas or liquid on the surface of a porous media (39). Adsorbents are highly porous solids. Since different molecules interact differently with the adsorbent, it is eventually possible to separate them (114). During the energy charging step (desorption process), heat added to the system is used to remove the gas or liquid molecules, for instance water, from the solid surface. When energy is needed the reverse reaction (adsorption) is promoted by adding water to the surface and the stored heat is released. An advantage of the sorption materials in comparison with PCMs is that, in the former, the energy release process can be designed to provide a cooling effect from the evaporator in summer or heating from the reactor in winter, whereas in the case of PCMs the fixed discharging temperature imposes a restriction on their application (17).

The desorption-adsorption storage process can be carried out in both open and closed configurations. Closed configurations mainly consist of two vessels, a reactor and a condenser-evaporator where water is stored. Higher temperatures for heating applications as well as lower temperatures for cooling can be reached in closed systems as compared to open systems. On the other hand, the pressure in the adsorption and desorption processes play a critical role. Thus, closed sorption systems present several benefits form a design point of view. Moreover, the energy density is lower compared to open systems since the adsorptive must be also stored. In open configurations, only water can be used as sorbate. Abedin et al. (115) developed a comparison between both types of thermochemical systems and concluded that the overall energy efficiency was 50 and $69 \%$ for closed and open systems, respectively.

Since the adsorption equilibrium is given by specific operating conditions (composition, temperature and pressure), by changing one of these parameters it is possible to regenerate the adsorbent. 
Thus, information about the adsorption equilibrium of the distinct species is vital to design and model adsorption processes (116). Most studied materials for energy storage by means of adsorption are zeolites and silica gel. New materials such as aluminophosphates (AlPOs) or metal-organic frameworks (MOFs) have been proposed $(17,117)$. Differences between zeolites and silica gel are related to their structure; while zeolites present a narrow pore size distribution determined by their crystalline structure, silica gel (which is composed of $99 \%$ of $\mathrm{SiO}_{2}$ ) has a larger range of pore sizes (39).

Silica gel is a desiccant widely used which allows working at a changing temperature bellow $100{ }^{\circ} \mathrm{C}$. One of the main drawbacks for silica gel is that water adsorption occurs at high relative pressures, leading to a low water exchange (17). In the case of zeolites, the main commercial types are Zeolite 4A, 5A, 10X and $13 \mathrm{X}$, which present a desorption temperature usually close to $150{ }^{\circ} \mathrm{C}(112,118)$ that limits their application to low temperature solar systems.

Adsorption of water vapour in both silica gel and zeolite compounds has been investigated experimentally in several works $(39,112)$. Experiments have shown an almost linear relation between air temperature and humidity at system exit when fixed adsorption conditions are considered (39). Thus, by increasing the humidity ratio $\left(\mathrm{kg} \mathrm{H}_{2} \mathrm{O} / \mathrm{kg}\right.$ material), air temperature is reduced in the energy releasing step. Regarding to the breakthrough curve, zeolite reaches its maximum outlet temperature which is kept almost constant until adsorption is over whereas in the case of silica gel the outlet temperature falls just after reaching its maximum (39).

Silica gel and zeolites have been tested in several research projects such as the HYDES and MODESTORE projects (2003-2012) (112, 119), which aim at the application of seasonal storage of solar thermal energy for space heating. For the material combination of silica gel and water, test results showed that the temperature lift is sufficient up to a water content in silica gel of about $13 \%$. On the other hand, water contents of less than $3 \%$ are not realistically achievable given the temperature attainable from flat plate solar collectors and the temperature of an available heat sink. Therefore, the material has to operate between these limits of water content $(3-13 \%)$. That means that the storage density of the material is significantly lower than initially expected. Lim et al. (120) built a lab scale closed loop system based on silica gel-water, in which the water uptake is approximately $29.8-56.7 \%$ the theoretical value. Sapienza et al. (121) studied the equilibrium and dynamics of the silica gel-water system by using Siogel, a commercial adsorbent. A water uptake of $12 \%$ was achieved. Deshmukh et al. (122) carried out thermal analysis of a closed silica gel-water system with the aim to develop a $18 \mathrm{kWh}$ storage system at an average temperature lift of $25^{\circ} \mathrm{C}$. Results showed that for these conditions $350 \mathrm{~kg}$ of adsorbent are necessary, with an energy storage density of $151 \mathrm{MJ} / \mathrm{m}^{3}$. Fernandes et al. (123) carried out simulations to evaluate a silica gelwater adsorption system which serves as TCES for a conventional solar system. Results indicated that annual energy savings up to $16 \%$ can be achieved in comparison with a conventional (water) storage system.

Results achieved with zeolites have shown a better performance due to its high water uptake compared to silica gel, which provides a high energy storage density. According to (112), Zeolite $13 \mathrm{X}$ has a higher water vapour uptake rate than Silica gel 490 (up to $32 \%$ of its dry weight). Thus, Zeolite 13X, which was identified as one of the best adsorbents, has been also tested within international projects, such as E-Hub project (2010-2014) or COMTES project (2012-2016). Hauer et al. (124) developed and tested an open adsorption system based on zeolite 13X which provides heat to a district heating facility. Reported results showed an energy storage density of $446 \mathrm{MJ} / \mathrm{m}^{3}$. A domestic hot water system with storage based on zeolite $13 \mathrm{X}$-water vapour was developed and tested by Köll et al. (125). Results demonstrated that an energy density of $640 \mathrm{MJ} / \mathrm{m}^{3}$ can be achieved, which allows achieving a solar fraction of $83.5 \%$ for both hot water and space heating. Tatsidjodoung et al. (126) also constructed a prototype using Zeolite $13 \mathrm{X} / \mathrm{H}_{2} \mathrm{O}$ as the reactive pair, which was validated by means of a $1 \mathrm{D}$ mathematical model. Results on discharging process showed that, with a $40 \mathrm{~kg}$ zeolite system, an average temperature lift of $38^{\circ} \mathrm{C}$ at the exit of zoolite vessel was attained during a period of 8 hours. As in similar prototypes, temperatures up to $180{ }^{\circ} \mathrm{C}$ were necessary to carry out the charging process. Analysis on the kinetics of the adsorption process for zeolite $13 \mathrm{X} / \mathrm{H}_{2} \mathrm{O}$ showed that slow reaction rates reduce the efficiency significantly albeit only below a threshold value for the kinetics coefficient, which for the selected conditions was $0.001[1 / \mathrm{s}](127)$.

Table 20 shows the main solid adsorbents tested as TSMs. As shown, most of them present a discharge temperature below $70{ }^{\circ} \mathrm{C}$ and therefore an efficient low-temperature terminal heating system, such as underfloor heating, is fundamental. Regarding the charging step, thermal energy sources at temperatures of up to $180^{\circ} \mathrm{C}$ for Zeolite and $95^{\circ} \mathrm{C}$ for Silica gel are needed. Energy density of these TSMs varies between 151 and $648 \mathrm{MJ} / \mathrm{m}^{3}$, which is quite high in comparison with water-based sensible heat systems, although an optimized infrastructure must be designed to reduce the volume of external equipment, such as tanks, pumps, blowers or heat exchangers.

4.3.1.2. Liquid absorption materials The sorption process in absorption materials is based on liquids, 
TABLE 20. TSMs. Solid physical adsorption. Properties

\begin{tabular}{|c|c|c|c|c|c|c|}
\hline Material & $\begin{array}{c}\text { Charge } \\
\text { temp. }\left({ }^{\circ} \mathrm{C}\right)\end{array}$ & $\begin{array}{l}\text { Discharge } \\
\text { temp. }\left({ }^{\circ} \mathbf{C}\right)\end{array}$ & $\begin{array}{c}\text { Storage density } \\
(\mathrm{kJ} / \mathrm{kg})\end{array}$ & $\begin{array}{c}\text { Volumetric storage } \\
\text { of material } \\
\left(\mathrm{MJ} / \mathrm{m}^{3}\right)\end{array}$ & $\begin{array}{c}\text { Volumetric storage } \\
\text { of prototype } \\
\left(\mathrm{MJ} / \mathrm{m}^{3}\right)\end{array}$ & Reference \\
\hline Silica gel $127 \mathrm{~B} / \mathrm{H}_{2} \mathrm{O}$ & 88 & $70-40$ & & 180 & 120 & $(112,119)$ \\
\hline Silica gel $/ \mathrm{H}_{2} \mathrm{O}$ & 95 & 25 & & 151 & & (122) \\
\hline \multirow[t]{2}{*}{ Zeolite $13 \mathrm{X} / \mathrm{H}_{2} \mathrm{O}$} & 180 & $65-55$ & & 648 & 208 & (112) \\
\hline & $20-120$ & $50-25$ & & 640.8 & & (125) \\
\hline Zeolite $5 \mathrm{~A} / \mathrm{H}_{2} \mathrm{O}$ & 103 & $53-36$ & 226.5 & 170 & 45 & $(128-130)$ \\
\hline Zeolite $13 \mathrm{XBF} / \mathrm{H}_{2} \mathrm{O}$ & 150 & $75-47$ & 399.6 & 277 & - & $(131,132)$ \\
\hline \multirow[t]{3}{*}{ Zeolite 4A/Air } & 180 & $35-10$ & & 576 & 432 & $(112)$ \\
\hline & 180 & $60-35$ & 474.8 & 346 & 215 & (118) \\
\hline & 230 & $60-35$ & 529.9 & 421 & 243 & (118) \\
\hline \multirow[t]{2}{*}{ Zeolite NaX/Air } & 180 & 57 & 787.5 & & 8 & (133) \\
\hline & 120 & 57 & 535.5 & & 5 & $(133)$ \\
\hline \multirow[t]{2}{*}{ Zeolite 13X/Air } & $120-160$ & $70-45$ & 336 & & 225 & $(129,130)$ \\
\hline & $130-180$ & 65 & & 446 & - & (124) \\
\hline
\end{tabular}

which absorb the gas (usually water vapour) releasing the previously stored energy. In general, liquid materials present higher energy density than solid adsorption materials. However, the energy storage of the prototype decreases significantly in absorption systems since the storage in tanks of liquid must be considered. An advantage of liquid absorption materials is that they can be pumped and used directly as heat transfer fluid, which helps reduce the heat losses. The main components of an absorption process for energy storage are: a desorber and an absorber, a condenser and an evaporator, two solution storage tanks (diluted and concentrated solution) and an absorbate storage tank (134). The operating principle of a stored renewable energy based on liquid absorption materials for heating is: when energy is available (solar in most of cases) an aqueous solution is pumped from the tank to the desorber, where energy input is used to increase the vapour pressure to reach equilibrium. After that, if energy input continues, the solution becomes concentrated as water vapour is released, which is condensed and stored in a water tank. When energy is needed, the concentrated solution is sent to the absorber while water is pumped from the storage tank to the evaporator producing water vapour, which is absorbed by the solution, being the released heat used for air-conditioning proposes.

Several materials have been tested in closed absorption cycles for TCES, such as $\mathrm{LiCl}, \mathrm{NaOH}$, $\mathrm{CaCl}_{2}$ and $\mathrm{LiBr}$. Main properties of these liquid absorption materials are shown in Table 21.

Based on $\mathrm{LiCl}$ salt absorption process, a thermochemical accumulator (TCA) was patented by Olsson et al. (147), which is currently at the commercial level for cooling applications. TCA consists of a

TABle 21. TSMs. Liquid absorption materials. Properties

\begin{tabular}{|c|c|c|c|c|c|c|}
\hline Material & $\begin{array}{c}\text { Charge temp. } \\
\left({ }^{\circ} \mathrm{C}\right)\end{array}$ & $\begin{array}{l}\text { Discharge } \\
\text { temp. } \\
\left({ }^{\circ} \mathrm{C}\right)\end{array}$ & $\begin{array}{l}\text { Storage density } \\
(\mathbf{k J} / \mathbf{k g})\end{array}$ & $\begin{array}{c}\text { Volumetric storage } \\
\text { of } \mathrm{material} \\
\left(\mathrm{MJ} / \mathrm{m}^{3}\right)\end{array}$ & $\begin{array}{c}\text { Volumetric storage of } \\
\text { prototype } \\
\left(\mathrm{MJ} / \mathrm{m}^{3}\right)\end{array}$ & Reference \\
\hline $\mathrm{LiCl}$ salt $/ \mathrm{H}_{2} \mathrm{O}$ & $46-87$ & $30-25$ & 736.8 & 911 & 306 & (112) \\
\hline \multirow[t]{3}{*}{$\mathrm{NaOH} / \mathrm{H}_{2} \mathrm{O}$} & $95-150$ & 70 & & 900 & 18 & $(112,135)$ \\
\hline & 95 & $32.8 / 56$ & & & & $(132,136-139)$ \\
\hline & & & & 1566 & & (140) \\
\hline \multirow[t]{2}{*}{$\mathrm{CaCl}_{2} / \mathrm{H}_{2} \mathrm{O}$} & $117-138$ & & & $382-1372$ & & $(98,141,142)$ \\
\hline & 130 & 165 & & 777.6 & & (143) \\
\hline \multirow[t]{3}{*}{$\mathrm{LiBr} / \mathrm{H}_{2} \mathrm{O}$} & $75-90$ & $40-30$ & & 921.6 & & $(134,144)$ \\
\hline & $\approx 40-110$ & & & & 368 & (145) \\
\hline & $\approx 40-110$ & 35 & & & $263-331$ & (146) \\
\hline
\end{tabular}


three-phase absorption process (solid, solution and vapour). The system works in batch mode. Water is desorbed from the solution in the charging mode and causes the solution saturation followed by the formation of solid crystals that fall under gravity into the vessel. In discharging mode, the saturated solution is pumped to a heat exchanger where it absorbs vapour coming from the evaporator, whose energy input is taken either from the environment (heating application) or from the building (cooling) (148). After that, the unsaturated solution passes though the crystals zone to reach saturation again before being stored. The condensation heat and released binding energy is transferred to the environment (cooling mode) or to the building (heating mode) (148). Laboratory test results showed an energy density for the material, $\mathrm{LiCl}$ salt, of $911 \mathrm{MJ} / \mathrm{m}^{3}$, although considering the entire TCES prototype the energy density results around $306 \mathrm{MJ} / \mathrm{m}^{3}$ (112). Hui et al. (149) evaluated seven absorption systems from an energy storage point of view. Results showed that the $\mathrm{LiCl} /$ $\mathrm{H}_{2} \mathrm{O}$ couple has an excellent storage capacity and efficiency, with the disadvantage of a high cost compared to other liquid absorbents (more than 14 times higher than $\mathrm{CaCl}_{2}$ ). Nevertheless, the high energy density of the LiCl-based system makes it an attractive choice from a storage capacity cost perspective resulting in one of the materials with lower cost per $\mathrm{kWh}$ stored $(\sim 6 € / \mathrm{kWh})(150)$.

In the case of the $\mathrm{NaOH}$ system, a prototype for heating has been tested (112). The system works as a heat pump in which the driving force is the difference of the vapour pressures above water and $\mathrm{NaOH}$ solution (soda lye). In this system configuration, contrary to the TCA described above, the tanks are separated and works as a continuous process. Test results showed a good kinetics at desorption temperatures of $120^{\circ} \mathrm{C}$. The energy density of the system and prototype are 900 and $18 \mathrm{MJ} / \mathrm{m}^{3}$ respectively, but this prototype can be highly improved with an optimized system design (112). Another test carried out by Weber et al. (135) showed that, including tanks and heat exchangers, the total energy density could be three times higher compared to traditional hot water storage at $70{ }^{\circ} \mathrm{C}$, and about six times higher for a tap temperature of $40{ }^{\circ} \mathrm{C}$ for low-temperature space heating. Fumey et al. (140) tested at lab-scale a conventional spiral fined tube heat exchanger where the absorbent $(\mathrm{NaOH})$ flows along the fin from top to bottom. A temperature lift of $35^{\circ} \mathrm{C}$ between the maximum absorbent temperature and the absorbate was measured, with a dilution from $50 \mathrm{wt} \%$ to $27 \mathrm{wt} \%$. During desorption a concentration lift from $25 \mathrm{wt} \%$ to $53 \mathrm{wt} \%$ is achieved, which translates into a theoretically energy density of $1566 \mathrm{MJ} / \mathrm{m}^{3}$.

$\mathrm{CaCl}_{2}$-based system behaviour was analysed by Davidson et al. (98) for its use as liquid desiccant in an atmospheric-pressure chiller. Solar energy is stored in a liquid $\mathrm{CaCl}_{2}$ volume by heating the solution and vaporizing $\mathrm{H}_{2} \mathrm{O}$. Energy is extracted as water is reabsorbed and/or the solution is cooled (142). The energy density of the material, which is dependent on how the tank is discharged, is $106 \mathrm{kWh} / \mathrm{m}^{3}$, similar to that of $\mathrm{LiCl}$ for the same operating parameters (98). Computational results showed that buoyancy driven flow induced by the presence of an immersed heat ex-changer is sufficient to heat and thermally stratify the storage fluid (142). Transport of mass across the density gradient between regions is limited by diffusion. Richter et al. (143) developed an experimental analysis on the absorption system based on $\mathrm{CaCl}_{2}$. In this work, storage is charged at $130{ }^{\circ} \mathrm{C}$ while the reverse reaction allows an energy discharge at $165^{\circ} \mathrm{C}$, which means a thermal upgrade of $35^{\circ} \mathrm{C}$ and a thermochemical storage density of approximately $777.6 \mathrm{MJ} / \mathrm{m}^{3}$. Esaki et al. (151) assessed experimentally the use of a chemical heat pump for upgrading waste heat during the hydration of $\mathrm{CaCl}_{2}$, which occurs within a packed bed reactor. A waste heat temperature upgrading from $100{ }^{\circ} \mathrm{C}$ up to $155^{\circ} \mathrm{C}$ was achieved with a performance coefficient of 0.48 .

Liquid absorbent $\mathrm{LiBr}$ was also tested by N'Tsoukpoe et al. $(134,144)$ as a promising candidate for long-term energy storage in buildings. An $8 \mathrm{kWh}$ energy storage prototype was built based on two storage tanks (water and solution: $88.5 \mathrm{~kg}$ ) and two falling films heat exchangers (desorber/absorber, condenser/evaporator) assembled to the reactor, which produces a heating power of $1 \mathrm{~kW}$. Several tests were carried out, focused in the charging process under practical conditions $\left(25^{\circ} \mathrm{C}-95^{\circ} \mathrm{C}\right.$ in the desorber and $10-30{ }^{\circ} \mathrm{C}$ in the condenser). It was demonstrated that a storage of $13 \mathrm{kWh}$ is possible when charging the prototype with a thermal power of 2-5 $\mathrm{kW}$. Discharging tests indicate absorption temperatures of about $30-40{ }^{\circ} \mathrm{C}$, which can be low for space heating. Xu et al. (145) proposed an absorption solar energy storage system which uses a $\mathrm{LiBr}$ solution. A stored energy density of $368 \mathrm{MJ} / \mathrm{m}^{3}$ was achieved. Perier-Muzet and Le Pierres (146) assessed the efficiency of a $\mathrm{LiBr} / \mathrm{H}_{2} \mathrm{O}$ absorption heat storage system integrated with a solar thermal facility for space heating. They analysed the impact of using a solution heat exchanger (SHX), which could improve both the energy efficiency and storage density up to $75 \%$ and $331 \mathrm{MJ} / \mathrm{m}^{3}$, respectively.

Another possibility is using liquid absorption materials for TCES in open systems. During absorption (discharging step) the concentrated solution is sent to an exchange surface where it gets in contact with an air stream. Humidity is removed from air and the solution becomes diluted by the absorption of vapour. During regeneration (reverse process) the solution is again concentrated by the mass and heat transfer with a hot air stream. In open absorption cycles the desiccants are regenerated against the ambient water vapour pressure (dew point of the 
ambient air) whereas the reference temperature is well above the ambient wet bulb temperature, thus open cycle regeneration requires lower regeneration temperatures and therefore higher collector efficiency (39). An open system based on $\mathrm{CaCl}_{2} / \mathrm{H}_{2} \mathrm{O}$ was tested by Bouché et al. (152), which, according to test results, could allow a thermal upgrade of approximately $65 \mathrm{~K}$.

\subsubsection{Chemical reactions}

4.3.2.1. Solid chemical reaction materials Energy storage by means of chemical reaction materials is based on using the heat obtained from an external source (e.g. solar) to drive an endothermic reaction. The products of the reaction are stored and, when energy is needed, brought together to induce the reversible reaction (exothermic). This group of energy storage materials present a higher energy storage density than previous materials. Main disadvantages could be a low chemical stability after cycling (loss on sorbent capacity) (129), slow kinetics associated to insufficient temperature rise in the reactor (153), and poor heat and mass transfer in gas-solid reactions (154). A number of salt hydrates have been analysed as potential candidates for TCES in buildings such as $\mathrm{MgSO}_{4} \cdot 7 \mathrm{H}_{2} \mathrm{O}, \mathrm{MgCl}_{2} \cdot 6 \mathrm{H}_{2} \mathrm{O}$ and $\mathrm{CaCl}_{2} \cdot 6 \mathrm{H}_{2} \mathrm{O}$. Table 22 shows the properties of main chemical reaction materials.

$\mathrm{MgSO}_{4} \cdot 7 \mathrm{H}_{2} \mathrm{O}$ is a promising energy storage material with a high energy density (155). A characterization of $\mathrm{MgSO}_{4} \cdot 7 \mathrm{H}_{2} \mathrm{O}$ as TSM was carried out by Bales et al. (112). The results obtained from TGA tests showed that the dehydration of $\mathrm{MgSO}_{4} \cdot 7 \mathrm{H} 2 \mathrm{O}$ occurs in three steps, namely, formation of $\mathrm{MgSO}_{4} \cdot 6 \mathrm{H}_{2} \mathrm{O}$, $\mathrm{MgSO}_{4} \cdot 0.2 \mathrm{H}_{2} \mathrm{O}$ and finally $\mathrm{MgSO}_{4}$.

By analysing the DSC curve (112), it is seen that the third step of dehydration (production of $\mathrm{MgSO}_{4}$ ) is exothermic, which is contrary to expectations in dehydration reactions (first and second step are endothermic). According to Ruiz-Agudo et al. (158), the reason for the endothermicity of the final reaction is the recrystallization of an amorphous precursor. During hydration, $\mathrm{MgSO}_{4}$ takes up water to form $\mathrm{MgSO}_{4} \cdot 6 \mathrm{H}_{2} \mathrm{O}$. If the releasing energy system works at atmospheric pressure, the $\mathrm{MgSO}_{4}$ could provide energy for heating applications at $25{ }^{\circ} \mathrm{C}$ while in a closed system at low pressure a small amount of energy could be released at $50{ }^{\circ} \mathrm{C}(159)$. At $50{ }^{\circ} \mathrm{C}$ the material is unable to take up water and deliver heat (159). These values are a limitation to the system application for heating purposes. Okhrimenko et al. (160) analysed dehydration of magnesium sulphate at low water vapour pressure and the reaction mechanism. Experimental results showed that the water content in the solid phase after dehydration depends on water vapour pressure and temperature. The equilibrium constant K was found to follow Van'tHoff equation with $\Delta \mathrm{H}=54.3 \mathrm{k} / \mathrm{mol} \mathrm{H}_{2} \mathrm{O}$ (160). According to Ferchaud et al. (161), the reaction kinetics for the dehydration reactions of $\mathrm{MgSO}_{4} \cdot 7 \mathrm{H}_{2} \mathrm{O}$ could reach a maximum for a water pressure of $50 \mathrm{~m}$ bar.

Zondag et al. (156) carried out several dynamic tests to analyse the dehydration kinetics of $\mathrm{MgSO}_{4} \cdot 7 \mathrm{H}_{2} \mathrm{O}, \mathrm{Al}_{2}\left(\mathrm{SO}_{4}\right) 3 \cdot 18 \mathrm{H}_{2} \mathrm{O}, \mathrm{CaCl}_{2} \cdot 6 \mathrm{H}_{2} \mathrm{O}$ and $\mathrm{MgCl}_{2} \cdot 6 \mathrm{H}_{2} \mathrm{O}$. To simulate the charging process, the first three materials were heated to $150{ }^{\circ} \mathrm{C}$ to provoke their dehydration while the $\mathrm{CaCl}_{2} \cdot 6 \mathrm{H}_{2} \mathrm{O}$ was dried at $130{ }^{\circ} \mathrm{C}$ to avoid side reactions. After that, to release the energy stored, the materials were hydrated with vapour. The authors observed that sulphates had a much lower temperature rise in the reactor than $\mathrm{CaCl}_{2} \cdot 6 \mathrm{H}_{2} \mathrm{O}$ and $\mathrm{MgCl}_{2} \cdot 6 \mathrm{H}_{2} \mathrm{O}$, which reached a temperature of around $50-68{ }^{\circ} \mathrm{C}$ depending on the reactor and vapour temperatures. In view of the results, $\mathrm{MgCl}_{2} \cdot 6 \mathrm{H}_{2} \mathrm{O}$ was selected as the most promising material for energy storage due to its relatively low charging temperature $\left(\geq 130{ }^{\circ} \mathrm{C}\right)$ and a discharging temperature sufficiently high to yield water heating $\left(\leq 60{ }^{\circ} \mathrm{C}\right)$, as well as its high energy density $\left(\sim 2 \mathrm{GJ} / \mathrm{m}^{3}\right)$ and low price $(<1 € / \mathrm{kg})(129)$. $\mathrm{MgCl}_{2} \cdot 6 \mathrm{H}_{2} \mathrm{O}$ was tested in a lab-scale prototype generating $150 \mathrm{~W}$ of thermal power (171 of material and a steam volume flow of $5101 / \mathrm{min}$ ), with an effective energy density of $0.5 \mathrm{GJ} / \mathrm{m}^{3}(129,156)$. According to Barreneche et al. (157) the energy involved in the $\mathrm{CaCl}_{2}$ dehydration process is $1470 \mathrm{MJ} / \mathrm{m}^{3}$.

4.3.2.1. Composite materials Composite materials, also called composite "salt porous matrix" (CSPM),

TABle 22. TSMs. Chemical reaction materials. Properties

\begin{tabular}{|c|c|c|c|c|c|c|}
\hline Material & $\begin{array}{c}\begin{array}{c}\text { Charge } \\
\text { temp. }\left({ }^{\circ} \mathrm{C}\right)\end{array} \\
\end{array}$ & $\begin{array}{l}\text { Discharge } \\
\text { temp. }\left({ }^{\circ} \mathbf{C}\right)\end{array}$ & $\begin{array}{c}\text { Storage density } \\
(\mathbf{k J} / \mathbf{k g})\end{array}$ & $\begin{array}{c}\text { Volumetric storage } \\
\text { of } \mathrm{material} \\
\left(\mathrm{MJ} / \mathrm{m}^{3}\right)\end{array}$ & $\begin{array}{c}\text { Volumetric storage } \\
\text { of prototype } \\
\left(\mathrm{MJ} / \mathrm{m}^{3}\right)\end{array}$ & Reference \\
\hline \multirow[t]{2}{*}{$\mathrm{MgSO}_{4} \cdot 7 \mathrm{H}_{2} \mathrm{O}$} & 150 & & & 1512 & - & $(112)$ \\
\hline & $275-60$ & $300-25$ & & $1800^{\mathrm{a}}$ & - & $(155)$ \\
\hline $\mathrm{MgCl}_{2} \cdot 6 \mathrm{H}_{2} \mathrm{O}$ & 130 & $64-50$ & & $\sim 2000$ & 500 & $(129,130,156)$ \\
\hline $\mathrm{CaCl}_{2} \cdot 6 \mathrm{H}_{2} \mathrm{O}$ & $165-190$ & 50.1 & & 1470 & & (157) \\
\hline
\end{tabular}

${ }^{\text {a }}$ This value is considering the hydration process at $25^{\circ} \mathrm{C}$. Energy supplied along the dehydration process is $2000 \mathrm{MJ} / \mathrm{m}^{3}$. This energy is approximately $88 \%$ of the energy density of the corresponding dehydration reaction (155). 
have been proposed to enhance the reaction kinetics and the poor heat and mass transfer in chemical reaction materials for energy storage. Composite materials usually consist of salt hydrates and an additive with a porous structure that works as host matrix with high thermal conductivity to improve the reaction rate and the heat release. Most studied materials to be used as additive are zeolites $(98,153,162)$, metal foam $(154,163)$, expanded graphite (164-166) and activated carbon (167). Table 23 shows the main properties of several composite materials for energy storage.

A composite material consisting of a reactive mixture of sodium sulphide/water and graphite additives was analysed by Lammak et al. (164), which was filled in a tube-in-tube heat exchanger and operated between high temperature source and a sink temperature of $30-40{ }^{\circ} \mathrm{C}$. The average heating and cooling power were about $3.79 \mathrm{~kW}$ and $0.23 \mathrm{~kW}$, respectively. They found that the amount of adsorbed water could be increased drastically by using the graphite-based composite materials. In another work, Mauran et al. (166) analysed a system composed by $\mathrm{SrBr}_{2}$ implemented with an expanded natural graphite as reactant and $\mathrm{H}_{2} \mathrm{O}$ as refrigerant fluid. The prototype reactor, with a total volume of $1 \mathrm{~m}^{3}$, was able to store $60 \mathrm{kWh}$ and $40 \mathrm{kWh}$ in heating and cooling modes respectively. Power released was $2.5 \mathrm{~kW}$ for heating and $4 \mathrm{~kW}$ for cooling applications, which was limited by a poor heat and mass transfer. Even though composite materials were expected to improve this issue, the transfer of heat at the inter-face between the consolidated composite and the wall of the exchanger posed a very strong limitation.

Mette et al. (153) proposed a new regeneration strategy for a thermochemical system based on a composite material composed by zeolite (matrix) and $\mathrm{CaCl}_{2}$, with the aim of reducing the regeneration temperature without affecting energy density. Simulation results showed that it is possible to reduce the regeneration temperature from $180^{\circ} \mathrm{C}$ to $130^{\circ} \mathrm{C}$ and therefore the process can be driven by solar thermal collectors. This implies a fractional energy saving higher than in the basic process without the proposed regeneration strategy. Wu et al. $(169,170)$ studied a $\mathrm{CaCL}_{2}$-silica gel composite material for energy storage. The results indicated that a $30 \mathrm{wt} \% \mathrm{CaCl}_{2}$ solution was the most stable and had the highest energy storage capacity: $822 \mathrm{MJ} / \mathrm{m}^{3}$ at a charging temperature of $90^{\circ} \mathrm{C}$. In another work, Jabbari-Hichri et al. (171) studied by means of TG/DCS analysis three supporting materials, silica-gel, alumina and bentonite, mixed with a $15 \%$ wt of $\mathrm{CaCl}_{2}$. Results showed that the $\mathrm{CaCl}_{2}$-silica gel composite material had the highest energy storage density, with a value of $746 \mathrm{~kJ} / \mathrm{kg}$.

\section{DISCUSSION OF RESULTS}

With the aim of comparing all reported TES materials for low-to-moderate temperature storage applications, Figure 3 illustrates the ranges of volumetric storage capacity of sensible, latent and thermochemical material groups.

Sensible storage capacity ranges depend on temperature ranges in which materials operate. For building applications, water shows the higher storage density due to its high volumetric specific heat, being able to storage $250 \mathrm{MJ} / \mathrm{m}^{3}$ for a temperature gradient of $60^{\circ} \mathrm{C}\left(25-85^{\circ} \mathrm{C}\right)$ in heating applications, and $42 \mathrm{MJ} / \mathrm{m}^{3}$ for a temperature gradient of $10^{\circ} \mathrm{C}$ $\left(17-7{ }^{\circ} \mathrm{C}\right)$ in cooling applications. These values should be considered as maximum reference values, which could be reduced according to final building application.

Latent heat storage ranges are based on heat of fusion of reported materials for the specific melting temperature. Also, ranges of all commercially available organic and inorganic materials are reported according to data published in Lizana et al. (172). Metal alloys, sugar alcohols and salt hydrates present the highest volumetric storage capacity of all studied PCMs, being situated between $124-419 \mathrm{MJ} / \mathrm{m}^{3}$, 281-442 MJ $/ \mathrm{m}^{3}$ and $218-514 \mathrm{MJ} / \mathrm{m}^{3}$, respectively.

Thermochemical heat storage ranges are drawn based on the energy storage density of materials without taking into account the whole systems'

TABLE 23. TSMs. CSPM. Properties

\begin{tabular}{|c|c|c|c|c|c|c|}
\hline Material & $\begin{array}{c}\text { Charge } \\
\text { temp. }\left({ }^{\circ} \mathrm{C}\right)\end{array}$ & $\begin{array}{l}\text { Discharge } \\
\text { temp. }\left({ }^{\circ} \mathbf{C}\right)\end{array}$ & $\begin{array}{c}\text { Storage density } \\
(\mathrm{kJ} / \mathrm{kg})\end{array}$ & $\begin{array}{c}\text { Volumetric storage } \\
\text { of material } \\
\left(\mathrm{MJ} / \mathrm{m}^{3}\right)\end{array}$ & $\begin{array}{c}\text { Volumetric storage } \\
\text { of prototype } \\
\left(\mathrm{MJ} / \mathrm{m}^{3}\right)\end{array}$ & Reference \\
\hline $\mathrm{Na}_{2} \mathrm{~S}$-graphite $/ \mathrm{H}_{2} \mathrm{O}$ & $95-80$ & & 8064 & & & (164) \\
\hline $\mathrm{Na}_{2} \mathrm{~S}$-cellulose $/ \mathrm{H}_{2} \mathrm{O}$ & $>83$ & $45-35$ & 3960 & & & $(168)$ \\
\hline $\mathrm{SrBr}_{2}-\mathrm{ENG} / \mathrm{H}_{2} \mathrm{O}$ & 80 & 35 & 1155.6 & 216 & & (166) \\
\hline Zeolite $4 \mathrm{~A}-\mathrm{CaCl}_{2} / \mathrm{H}_{2} \mathrm{O}$ & 130 & 25 & 3600 & 900 & & $(98,153,162)$ \\
\hline $\mathrm{CaCl}_{2}(30 \% \mathrm{wt})$-silica gel & 90 & 60 & 1020 & 822 & & (169) \\
\hline $\mathrm{CaCl}_{2}(43 \% \mathrm{wt})$-silica gel & 80 & 30 & & 760 & & $(170)$ \\
\hline $\mathrm{CaCl}_{2}(15 \% \mathrm{wt})$-silica gel & & & 746 & & & $(171)$ \\
\hline
\end{tabular}




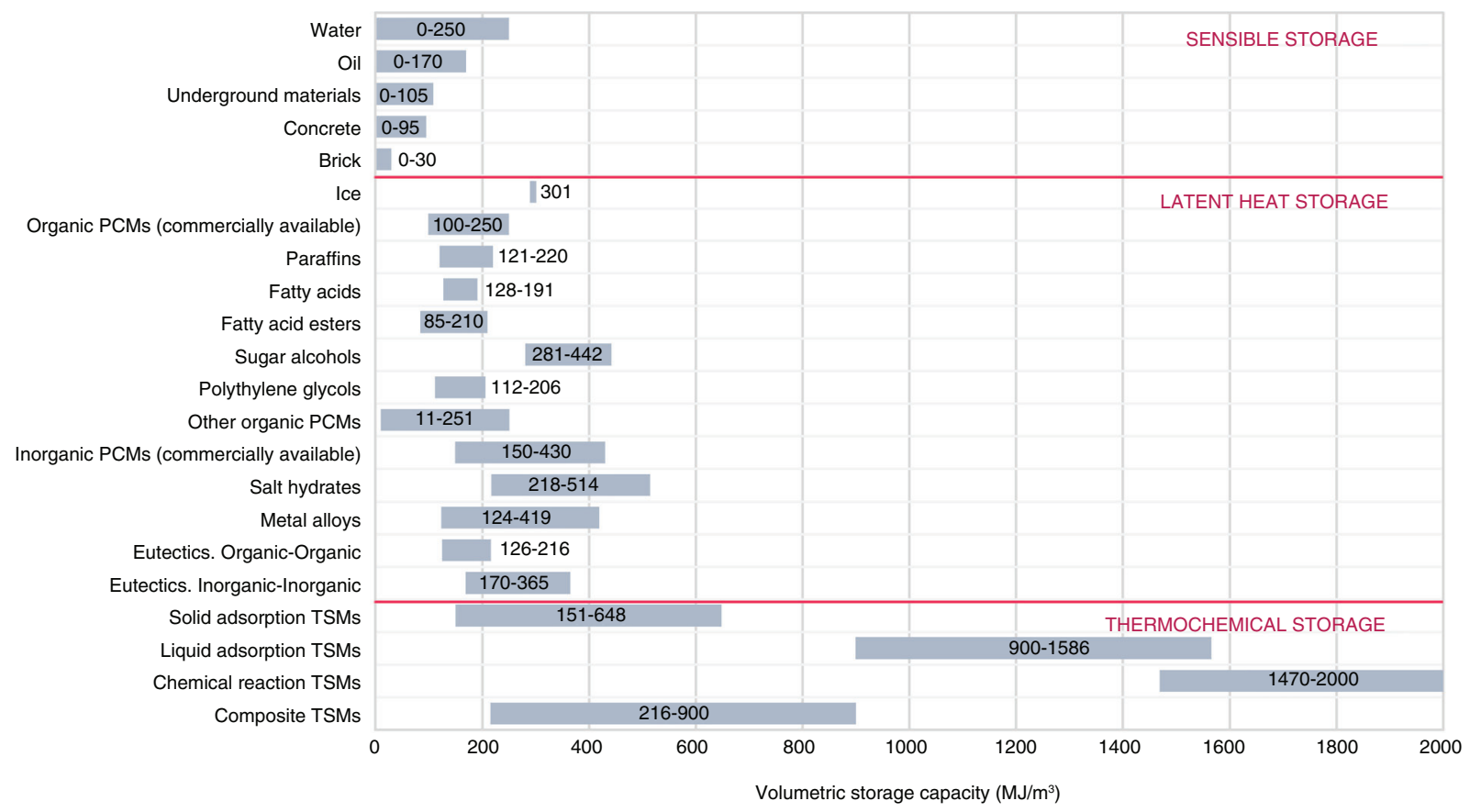

FIGURE 3. Comparison of TES ranges of reported materials for low-to-moderate temperature applications

components (tanks and heat exchangers), which can significantly reduce the effective material storage density by more than a 50\% (16). Liquid absorption and chemical reaction processes show the highest energy storage capacity, being in the range of $900-1566 \mathrm{MJ} / \mathrm{m}^{3}$ and $1470-2000 \mathrm{MJ} / \mathrm{m}^{3}$, respectively.

Figure 4 illustrates volumetric latent heat capacity and melting temperature of all reported pure compounds and mixtures of PCMs tested in the literature by different authors: organics such as paraffins, fatty acids, esters, sugar alcohols, polyethylene glycols; inorganics such as salt hydrates and metals; and eutectics. Sensible TES processes in water for heating and cooling applications are also illustrated as a reference maximum value (yellow line).

This figure allows identifying the PCMs with the highest volumetric storage capacity and with an appropriate melting temperature for heating, cooling and comfort applications. For cooling applications, those with the highest potential are lithium chlorate trihydrate $\left[\mathrm{LiCiO}_{3} \cdot 3 \mathrm{H}_{2} \mathrm{O}\right]$ and the eutectic mixture of lithium nitrate trihydrate and zinc nitrate hexahydrate $\left[\mathrm{LiNO}_{3} \cdot 3 \mathrm{H}_{2} \mathrm{O}\right.$ and $\mathrm{Zn}\left(\mathrm{NO}_{3}\right)_{2} \cdot 6 \mathrm{H}_{2} \mathrm{O}$ ]. For comfort applications, lithium nitrate trihydrate $\left[\mathrm{LiNO}_{3} \cdot 3 \mathrm{H}_{2} \mathrm{O}\right]$ and the eutectic mixture of $\left[\mathrm{CaCl}_{2}+\right.$ $\mathrm{NaCl}+\mathrm{KCl}+\mathrm{H}_{2} \mathrm{O}$ ] seem promising to reduce overheating; and for heating and solar applications, sodium sulphide pentahydrate $\left[\mathrm{Na}_{2} \mathrm{~S} \cdot 5 \mathrm{H}_{2} \mathrm{O}\right]$, ammonium aluminium sulphate dodecahydrate $\left[\left(\mathrm{NH}_{4}\right)\right.$ $\mathrm{Al}\left(\mathrm{SO}_{4}\right)_{2} \cdot 12 \mathrm{H}_{2} \mathrm{O}$ ], barium hydroxide octahydrate $\left[\mathrm{Ba}(\mathrm{OH})_{2} \cdot 8 \mathrm{H}_{2} \mathrm{O}\right]$ and sodium hydroxide monohydrate $\left[\mathrm{NaOH} \cdot \mathrm{H}_{2} \mathrm{O}\right]$ stand out as the best available compounds, with a storage density between 450 and $514 \mathrm{MJ} / \mathrm{m}^{3}$. On the other hand, despite the high volumetric storage capacity of some metal alloys and sugar alcohols, their melting temperatures are high, which reduces their applicability for building solutions.

Figure 5 shows the volumetric storage capacity and melting temperature of all reported TSMs tested in the literature by different authors: solid physical adsorption, liquid absorption, chemical reaction and composite materials. Sensible TES processes in water for heating and cooling applications are also illustrated as a reference maximum value (yellow line).

TSMs have the highest energy storage density considering all sensible and latent tested compounds. However, these values do not account for the volume required of other components for their implementation, such as tanks and heat exchangers. In the case of gas-solid thermochemical systems, the internal porosity of solids and the particle packing fraction (173) should be taken into account for the total infrastructure volume estimation. Furthermore, they often do not satisfy all requirements for building applications. Low discharge capacities as a consequence of a slow kinetics, variable thermal power over time, high cost and long thermal stability under cycling are common issues to be overcome. Among all tested materials for small-scale applications in buildings, Zeolite 13X (solid physical adsorption), $\mathrm{LiCl}$ salt/ $\mathrm{H}_{2} \mathrm{O}$ (liquid absorption), $\mathrm{LiBr} / \mathrm{H}_{2} \mathrm{O}$ (liquid absorption) and $\mathrm{MgCl}_{2} \cdot 6 \mathrm{H}_{2} \mathrm{O}$ (chemical reaction) 


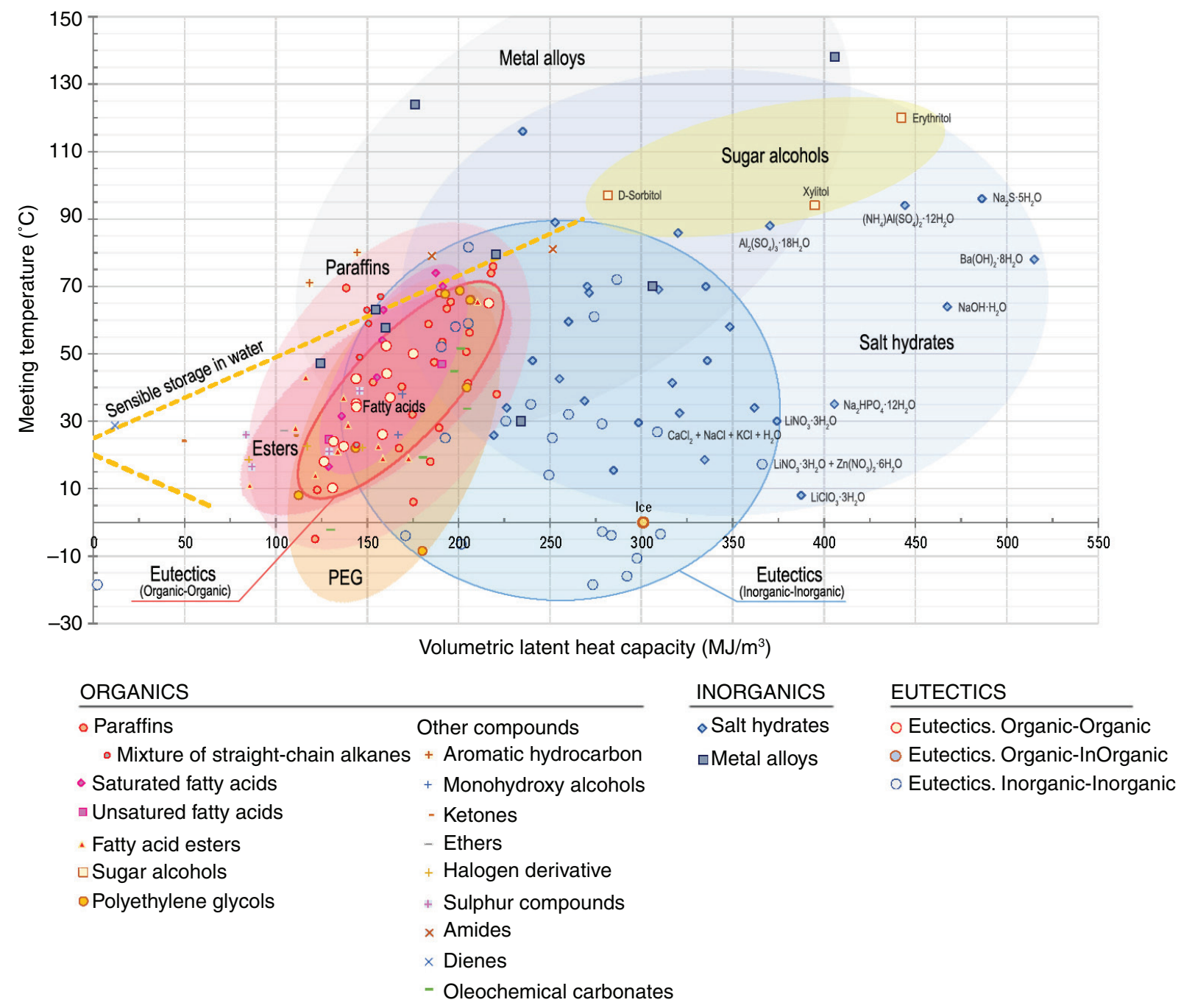

FIGURE 4. Volumetric storage capacity of latent heat storage materials compared with sensible storage in water.

have the highest potential for building applications according to the results obtained by different authors $(112,125,129,130,145,146,156)$. Considering all reported properties, $\mathrm{MgCl}_{2} \cdot 6 \mathrm{H}_{2} \mathrm{O}$ is the most promising material for energy storage due to its high energy density $\left(\sim 2 \mathrm{GJ} / \mathrm{m}^{3}\right)$, relatively low charging temperature $\left(\geq 130^{\circ} \mathrm{C}\right)$, a discharging temperature sufficiently high to produce water heating $\left(\leq 60{ }^{\circ} \mathrm{C}\right)$ and low price $(<1 € / \mathrm{kg})$. The tested prototype based on $\mathrm{MgCl}_{2} \cdot 6 \mathrm{H}_{2} \mathrm{O}$ in the E-HUB (2010-2014) achieved an effective energy density of $0.5 \mathrm{GJ} / \mathrm{m}^{3}(129,130)$. However, additional research efforts are required to improve its chemical stability under conditions for seasonal storage (129).

\section{CONCLUSIONS}

This paper reviews an extensive number of thermal energy storage (TES) materials technically developed for low-to-moderate temperature storage applications in buildings with the aim of identifying best available solutions. The results show that:

- Up to $21^{\circ} \mathrm{C}$, for cooling applications, salt hydrates and their eutectics show the highest volumetric storage capacity, being placed in an energy density range of $300-387 \mathrm{MJ} / \mathrm{m}^{3}$, with melting points between $21^{\circ} \mathrm{C}$ and $5{ }^{\circ} \mathrm{C}$. Lithium chlorate trihydrate $\left[\mathrm{LiCiO}_{3} \cdot 3 \mathrm{H}_{2} \mathrm{O}\right]$ and the eutectic mixture of lithium nitrate trihydrate and zinc nitrate hexahydrate $\left[\mathrm{LiNO}_{3} \cdot 3 \mathrm{H}_{2} \mathrm{O}\right.$ and $\left.\mathrm{Zn}\left(\mathrm{NO}_{3}\right)_{2} \cdot 6 \mathrm{H}_{2} \mathrm{O}\right]$ are the best available compounds, with a storage density of 387 and $366 \mathrm{MJ} / \mathrm{m}^{3}$, respectively. Also, latent heat storage in ice has a high potential due to the high availability, low cost and high storage capacity of $301 \mathrm{MJ} / \mathrm{m}^{3}$. However, its melting temperature could be lower for some domestic applications. In addition, sensible water storage remains currently as the most widely-used storage method for cooling purposes, with a volumetric 


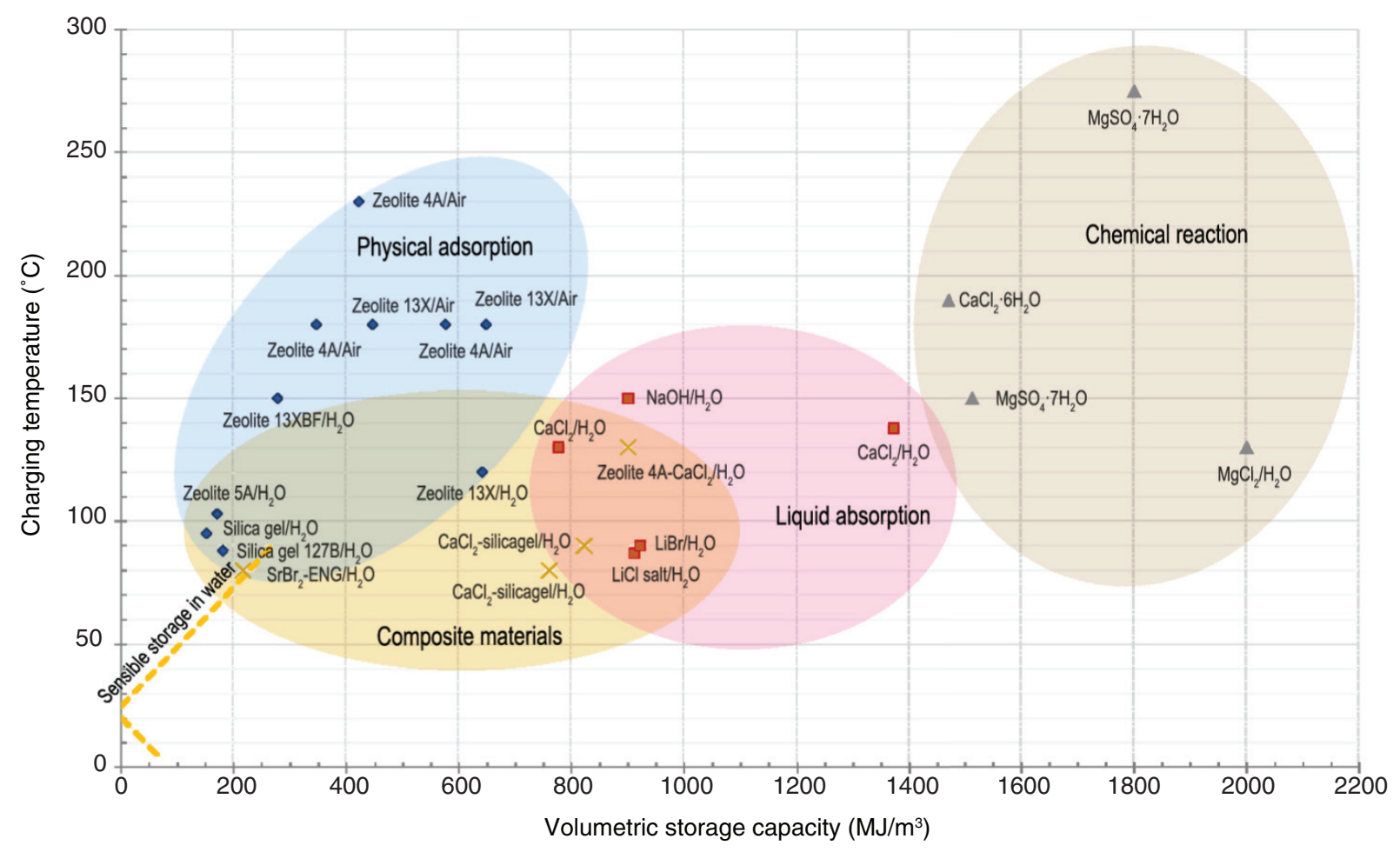

SORPTION

- Physical adsorption of solid materials

- Absorption of liquid materials

\section{CHEMICAL REACTIONS}

Chemical reaction materials

$\times$ Chemical reaction based on CSPM

FIGURE 5. Volumetric storage capacity of thermochemical storage materials compared with sensible storage in water.

storage capacity of $42 \mathrm{MJ} / \mathrm{m}^{3}$ for a temperature gradient of $10^{\circ} \mathrm{C}\left(17-7^{\circ} \mathrm{C}\right)$.

- Between $22^{\circ} \mathrm{C}$ and $28^{\circ} \mathrm{C}$, for comfort conditions, salt hydrates and their eutectics show the highest volumetric storage capacity, with a range between 250 and $373 \mathrm{MJ} / \mathrm{m}^{3}$. Lithium nitrate trihydrate $\left[\mathrm{LiNO}_{3} \cdot 3 \mathrm{H}_{2} \mathrm{O}\right]$ and the eutectic mixture of $\left[\mathrm{CaCl}_{2}+\mathrm{NaCl}+\mathrm{KCl}+\mathrm{H}_{2} \mathrm{O}\right]$ are the greatest potential compounds for comfort purposes. They seem good suited to reduce overheating due to their very high storage density of 373 and $308 \mathrm{MJ} / \mathrm{m}^{3}$, and their melting points of $30{ }^{\circ} \mathrm{C}$ and $26.8^{\circ} \mathrm{C}$, respectively. However, most of salt hydrates are characterized by low thermal reliability for long-operation periods, phase segregation and subcooling, which limit their effective application.

- Over $30^{\circ} \mathrm{C}$, for hot water, space heating and solar applications, a group of salt hydrates are identified as the best available compounds, with a storage density between 450 and $514 \mathrm{MJ} / \mathrm{m}^{3}$. They consist of sodium sulphide pentahydrate $\left[\mathrm{Na}_{2} \mathrm{~S} \cdot 5 \mathrm{H}_{2} \mathrm{O}\right]$, ammonium aluminium sulphate dodecahydrate $\left[\left(\mathrm{NH}_{4}\right) \mathrm{Al}\left(\mathrm{SO}_{4}\right)_{2} \cdot 12 \mathrm{H}_{2} \mathrm{O}\right]$, barium hydroxide octahydrate $\left[\mathrm{Ba}(\mathrm{OH})_{2} \cdot 8 \mathrm{H}_{2} \mathrm{O}\right]$ and sodium hydroxide monohydrate $\left[\mathrm{NaOH} \cdot \mathrm{H}_{2} \mathrm{O}\right]$, with a melting temperatures of $96{ }^{\circ} \mathrm{C}, 95^{\circ} \mathrm{C}$, $78{ }^{\circ} \mathrm{C}$ and $64{ }^{\circ} \mathrm{C}$, respectively. However, as for other salt hydrates, they present important drawbacks for their efficient implementation. This is why water and underground materials remain as the most widely-used storage mediums due to their high sensible storage capacity and moderate thermal diffusivity. Water is able to store $250 \mathrm{MJ} / \mathrm{m}^{3}$ for a temperature gradient of $60^{\circ} \mathrm{C}$ $\left(25-85{ }^{\circ} \mathrm{C}\right)$. Besides, underground solutions show the benefit of using the ground as insulation, thus they store thermal energy more efficiently than above-ground solutions. Regarding thermochemical storage materials, despite their high energy densities and high storage ability for long-term storage periods due to their negligible heat losses, there is no available material that currently satisfies all requirements for commercial deployment. Among all tested materials, the most promising are Zeolite 13X (solid physical adsorption), $\mathrm{LiCl}$ salt $/ \mathrm{H}_{2} \mathrm{O}$ (liquid absorption), $\mathrm{LiBr} / \mathrm{H}_{2} \mathrm{O}$ (liquid absorption), and, above all, $\mathrm{MgCl}_{2} \cdot 6 \mathrm{H}_{2} \mathrm{O}$ (chemical reaction) due to its high volumetric storage density $\left(2 \mathrm{GJ} / \mathrm{m}^{3}\right.$ for the 
material and $0.5 \mathrm{GJ} / \mathrm{m}^{3}$ for the final prototype), relatively low charging temperature $\left(\geq 130^{\circ} \mathrm{C}\right)$, high discharging temperature $\left(\leq 60{ }^{\circ} \mathrm{C}\right)$ and low price $(<1 € / \mathrm{kg})$. However, it suffers from a progressive decay of reactivity under cycling. Thermochemical energy storage is not mature enough for building applications. Additional research efforts are needed to optimize operation conditions, multi-cycling efficiency, material cost and systems design. In addition, thermochemical solutions require different tanks and heat exchangers, which reduce significantly the effective storage density and increase final costs.

Future research activities should be focused on experimental campaigns to validate and improve the performance of those materials with the highest potential with the aim of achieving practical solutions with high storage capacity, high thermal reliability and stability for long-operation periods, and competitive cost.

\section{ACKNOWLEDGMENTS}

This work was supported as part of the ClimACT project (SOE1/P3/P0429EU) within the Interreg Sudoe Programme, funded by European Regional Development Funds. The authors gratefully acknowledge the financial support via two predoctoral contracts granted to Francisco Jesús Lizana Moral (FPU14/06583) from the Spanish Ministry of Education, Culture and Sport; and to Carlos Ortiz Dominguez (BES-2015-0703149) from the Spanish Ministry of Economy and Competitiveness (contracts CTQ2014-52763-C2-2-R and CTQ201783602-C2-2R, FEDER funds).

\section{REFERENCES}

1. Pielichowska, K.; Pielichowski, K. (2014) Phase change materials for thermal energy storage. Prog. Mater. Sci. 30, 67-123. https://doi.org/10.1016/j.pmatsci.2014.03.005.

2. Mehling, H.; Cabeza, L.F. (2008) Heat and cold storage with PCM. An up to date introduction into basics and applications, Springer.

3. Abhat, A. (1980) Short term thermal energy storage. Rev. Phys. Appliquée. 15, 477-501. https://doi.org/10.1051/rphysap:01980001503047700.

4. Abhat, A. (1983) Low temperature latent heat thermal energy storage: Heat storage materials. Sol. Energy. 30, 313-332.

5. Lane, G.A. (1980) Low temperature heat storage with phase change materials. Int. J. Ambient Energy. 1, 155-168. https:// doi.org/10.1080/01430750.1980.9675731

6. Telkes, M. (1980) Thermal energy storage in salt hydrates. Sol. Energy Mater. 2, 381-393. https://doi.org/10.1016/ 0165-1633(80)90033-7.

7. Telkes, M. Thermal storage in salt-hydrates. in: Sol. Mater. Sci., Academic Press, : pp. 377-404.

8. Schröder, J.; Gawron, K. (1981) Latent heat storage. Int. J. Energy Res. 5, 103-109.

9. Naumann, R.; Emons, H.H. (1989) Results of thermal analysis for investigation of salt hydrates as latent heatstorage materials. J. Therm. Anal. 35, 1009-1031. https://doi. org/10.1007/BF02057256.
10. Sharma, A.; Tyagi, V.V.; Chen, C.R.; Buddhi, D. (2009) Review on thermal energy storage with phase change materials and applications. Renew. Sustain. Energy Rev. 13, 318-345. https://doi.org/10.1016/j.rser.2007.10.005.

11. Sharma, R.K.; Ganesan, P.; Tyagi, V. V.; Metselaar, H.S.C.; Sandaran, S.C. (2015) Developments in organic solid-liquid phase change materials and their applications in thermal energy storage. Energy Convers. Manag. 95, 193-228. https:// doi.org/10.1016/j.enconman.2015.01.084.

12. Cabeza, L.F.; Castell, A.; Barreneche, C.; de Gracia, A.; Fernández, A. I. (2011) Materials used as PCM in thermal energy storage in buildings: A review. Renew. Sustain. Energy Rev. 15, 1675-1695. https://doi.org/10.1016/j.rser. 2010.11.018.

13. Tyagi, V.V.; Buddhi, D. (2007) PCM thermal storage in buildings: A state of art. Renew. Sustain. Energy Rev. 11, 1146-1166. https://doi.org/10.1016/j.rser.2005.10.002.

14. Tyagi, V.V.; Kaushik, S.C.; Tyagi, S.K.; Akiyama, T. (2011) Development of phase change materials based microencapsulated technology for buildings: A review. Renew. Sustain. Energy Rev. 15, 1373-1391. https://doi.org/10.1016/j.rser. 2010.10.006

15. Zhou, D.; Zhao, C.Y.; Tian, Y. (2012) Review on thermal energy storage with phase change materials (PCMs) in building applications. Appl. Energy. 92, 593-605. https:// doi.org/10.1016/j.apenergy.2011.08.025.

16. N'Tsoukpoe, K.E.; Liu, H.; Le Pierrès, N.; Luo, L. (2009) A review on long-term sorption solar energy storage. Renew. Sustain. Energy Rev. 13, 2385-2396. https://doi. org/10.1016/j.rser.2009.05.008.

17. Yu, N.; Wang, R.Z.; Wang, L.W. (2013) Sorption thermal storage for solar energy. Prog. Energy Combust. Sci. 39, 489-514. https://doi.org/10.1016/j.pecs.2013.05.004 Review.

18. de Gracia, A.; Cabeza, L.F. (2015) Phase change materials and thermal energy storage for buildings. Energy Build. 103, 414 419. https://doi.org/10.1016/j.enbuild.2015.06.007.

19. Kenisarin, M.; Mahkamov, K. (2007) Solar energy storage using phase change materials. Renew. Sustain. Energy Rev. 11, 1913-1965. https://doi.org/doi:10.1016/j.rser.2006. 05.005 .

20. Kenisarin, M.; Mahkamov, K. (2016) Salt hydrates as latent heat storage materials:Thermophysical properties and costs. Sol. Energy Mater. Sol. Cells. 145, 255-286. https://doi.org/10.1016/j.solmat.2015.10.029.

21. Alva, G.; Liu, L.; Huang, X.; Fang, G. (2017) Thermal energy storage materials and systems for solar energy applications. Renew. Sustain. Energy Rev. 68, 693-706. https:// doi.org/10.1016/j.rser.2016.10.021

22. Alva, G.; Lin, Y.; Liu, L.; Fang, G. (2017) Synthesis, characterization and applications of microencapsulated phase change materials in thermal energy storage: A review. Energy Build. 144, 276-294. https://doi.org/10.1016/j.enbuild.2017. 03.063 .

23. Kalaiselvam, S.; Parameshwaran, R. (2014) Thermal energy storage technologies for sustainability: systems design, assessment, and applications, 1st ed.Academic Press.

24. Navarro, L.; de Gracia, A.; Niall, D.; Castell, A.; Browne, M.; McCormack, S.J.; Griffiths, P.; Cabeza, L.F. (2015) Thermal energy storage in building integrated thermal systems: A review. Part 2. Integration as passive system. Renew. Energy. 85, 1334-1356. https://doi.org/10.1016/j. renene.2015.06.064

25. Navarro, L.; Gracia, A. De; Niall, D.; Castell, A.; Browne, M.; Mccormack, S.J.; Grif, P.; Cabeza, L.F (2016) Thermal energy storage in building integrated thermal systems: A review. Part 1. Active storage systems. Renew. Energy. 88, 526-547. https://doi.org/10.1016/j.renene.2015.11.040.

26. Lizana, J.; Chacartegui, R.; Barrios-Padura, Á.; Ortiz, C.; Vilches, A. Evaluation of thermal energy storage technologies for heating, cooling and hot water applications road to zero energy buildings. in: 11th Conf. Sustain. Dev. Energy, Water Environ. Syst. SDEWES 2016, Lisbon.: pp. 1-16.

27. Heier, J.; Bales, C.; Martin, V. (2015) Combining thermal energy storage with buildings - a review. Renew. Sustain. Energy Rev. 42, 1305-1325. https://doi.org/10.1016/j.rser. 2014.11.031. 
28. Waqas, A.; Ud Din, Z. (2013) Phase change material (PCM) storage for free cooling of buildings-A review. Renew. Sustain. Energy Rev. 18, 607-625. https://doi.org/10.1016/j. rser.2012.10.034.

29. Hasan, A.; McCormack, S.J.; Huang, M.J.; Norton, B. (2014) Energy and cost saving of a photovoltaic-phase change materials (PV-PCM) System through temperature regulation and performance enhancement of photovoltaics. Energies. 7, 1318-1331. https://doi.org/10.3390/en7031318.

30. Huang, M.J.; Eames, P.C.; Norton, B. (2004) Thermal regulation of building-integrated photovoltaics using phase change materials. Int. J. Heat Mass Transf. 47, 2715-2733. https://doi.org/10.1016/j.ijheatmasstransfer.2003.11.015.

31. Huang, M.J.; Eames, P.C.; Norton, B. (2006) Phase change materials for limiting temperature rise in building integrated photovoltaics. Sol. Energy. 80, 1121-1130. https:// doi.org/10.1016/j.solener.2005.10.006

32. Hyman, L. Overview. in: Sustain. Therm. Storage Syst. Planning, Des. Oper., The McGraw-Hill, : pp. 1-23.

33. International Energy Agency (2013) Transition to Sustainable Buildings. Strategies and Opportunities to 2050, OECD/IEA. https://doi.org/10.1787/9789264202955-en.

34. Lizana, J.; Ortiz, C.; Soltero, V.M.; Chacartegui, R. (2017) District heating systems based on low-carbon energy technologies in Mediterranean areas. Energy. 120, 397-416. https://doi.org/10.1016/j.energy.2016.11.096.

35. Lizana, J.; Chacartegui, R.; Barrios-Padura, A.; Ortiz, C. (2017) Advanced low-carbon energy measures based on thermal energy storage in buildings: A review. Renew. Sustain. Energy Rev. 82, 3705-3749. https://doi.org/10.1016/ j.rser.2017.10.093

36. Ståhl, F. Influence of thermal mass on the heating and cooling demands of a building unit, $\mathrm{PhD}$ thesis. Sweden: Chalmers University of Technology, 2009. http://publications. lib.chalmers.se/publication/102603-influence-of-thermalmass-on-the-heating-and-cooling-demands-of-a-building-unit $\% 5$ Cnhttp://publications.lib.chalmers.se/records/ fulltext/102603.pdf.

37. Harikrishnan, S.; Deenadhayalan, M.; Kalaiselvam, S. (2014) Experimental investigation of solidification and melting characteristics of composite PCMs for building heating application. Energy Convers. Manag. 86, 864-872. https://doi.org/10.1016/j.enconman.2014.06.042.

38. Tatsidjodoung, P.; Le Pierrès, N.; Luo, L. (2013) A review of potential materials for thermal energy storage in building applications. Renew. Sustain. Energy Rev. 18, 327-349. https://doi.org/10.1016/j.rser.2012.10.025.

39. Paksoy, H.Ö. (2007) Thermal Energy Storage for Sustainable Energy Consumption

40. ISO 10456:2007 (2007). Building materials and products. Hygrothermal properties. Tabulated design values and procedures for determining declared and design thermal values.

41. Tudela, F. (1982) Ecodiseño, Universidad Autónoma Metropolitana de Xochimilco.

42. Asan, H.; Sancaktar, Y.S. (1998) Effects of wall's thermophysical properties on time lag and decrement factor. Energy Build. 28, 159-166. https://doi.org/10.1016/ S0378-7788(98)00007-3.

43. Onder, E.; Sarier, N.; Cimen, E. (2007) Encapsulation of phase change materials by complex coacervation to improve thermal performances of woven fabrics. Thermochim. Acta. 467, 63-72. https://doi.org/10.1016/j.tca.2007.11.007.

44. Hasnain S.M. (1998) Review on sustainable thermal energy storage technologies, Part I: heat storage materials and techniques. Energy Convers. Manag. 39, 1127-1138. https:// doi.org/10.1016/S0196-8904(98)00025-9.

45. Konuklu, Y.; Paksoy, H.O.; Unal, M. (2015) Nanoencapsulation of n-alkanes with poly(styrene-coethylacrylate) shells for thermal energy storage. Appl. Energy. 150, 335-340. https://doi.org/10.1016/j. apenergy.2014.11.066.

46. Zalba, B.; Marín, J.M.; Cabeza, L.F.; Mehling, H. (2003) Review on thermal energy storage with phase change: materials, heat transfer analysis and applications. Appl. Therm. Eng. 23, 251-283. https://doi.org/10.1016/S13594311(02)00192-8.
47. Schmidt, R.; Griesbaum, K.; Behr, A.; Biedenkapp, D.; Voges, H.-W.; Garbe, D.; Paetz, C.; Collin, G.; Mayer, D.; Höke, H. Hydrocarbons. in: Ullmann's Encycl. Ind. Chem. Wiley-VCH.

48. Mehling, H.; Cabeza, L.F. Solid-liquid phase change materials. in: Heat Cold Storage with PCM. An up to Date Introd. into Basics Appl. Springer: pp. 11-55.

49. Nagano, K.; Mochida, T.; Takeda, S.; Domański, R.; Rebow, M. (2003) Thermal characteristics of manganese (II) nitrate hexahydrate as a phase change material for cooling systems. Appl. Therm. Eng. 23, 229-241. https://doi. org/10.1016/S1359-4311(02)00161-8.

50. Dimaano, M.N.R.; Watanabe, T. (2002) The capric-lauric acid and pentadecane combination as phase change material for cooling applications. Appl. Therm. Eng. 22, 365-377. https://doi.org/10.1016/S1359-4311(01)00095-3.

51. Koschenz, M.; Lehmann, B. (2004) Development of a thermally activated ceiling panel with PCM for application in lightweight and retrofitted buildings. Energy Build. 36, 567-578. https://doi.org/10.1016/j.enbuild.2004.01.029.

52. Paris, J.; Falardeau, M.; Villeneuve, C. (1993) Thermal storage by latent heat: a viable option for energy conservation in buildings. Energy Sources. 15, 85-93.

53. He, F.; Wang, X.; Wu, D. (2014) New approach for sol-gel synthesis of microencapsulated n-octadecane phase change material with silica wall using sodium silicate precursor. Energy. 67, 223-233. https://doi.org/10.1016/j.energy. 2013.11.088

54. Sasaguchi, K.; Viskanta, R. (1989) Phase Change Heat Transfer During Melting and Resolidification of Melt Around Cylindrical Heat Source(s)/Sink(s). J. Energy Resour. Technol. 111, 43-49. https://doi.org/10.1115/1.3231400.

55. Mohaddes, F.; Islam, S.; Shanks, R.; Fergusson, M.; Wang, L.; Padhye, R. (2014) Modification and evaluation of thermal properties of melamine-formaldehyde/ n-eicosane microcapsules for thermo-regulation applications. Appl. Therm. Eng. 71, 11-15. https://doi.org/10.1016/j.applthermaleng.2014.06.016.

56. Sari, A.; Karaipekli, A. (2007) Thermal conductivity and latent heat thermal energy storage characteristics of paraffin/expanded graphite composite as phase change material. Appl. Therm. Eng. 27, 1271-1277. https://doi.org/10.1016/j. applthermaleng.2006.11.004

57. Zalba, B.; Marín, J.M.; Cabeza, L.F.; Mehling, H. (2004) Free-cooling of buildings with phase change materials. Int. J. Refrig. 27, 839-849. https://doi.org/10.1016/j.ijrefrig.2004. 03.015 .

58. Marín, J.M.; Zalba, B.; Cabeza, L.F.; Mehling, H. (2005) Improvement of a thermal energy storage using plates with paraffin-graphite composite. Int. J. Heat Mass Transf. 48, 2561-2570. https://doi.org/10.1016/j.ijheatmasstransfer.2004. 11.027 .

59. Cabeza, L.F.; Mehling, H.; Hiebler, S.; Ziegler, F. (2002) Heat transfer enhancement in water when used as PCM in thermal energy storage. Appl. Therm. Eng. 22, 1141-1151. https://doi.org/10.1016/S1359-4311(02)00035-2.

60. Mettawee, E.-B.S.; Assassa, G.M.R. (2007) Thermal conductivity enhancement in a latent heat storage system. Sol. Energy. 81, 839-845.

61. Dincer, I.; Rosen, M.A. (2010) Thermal Energy Storage: Systems and Applications, $2^{\mathrm{a} J o h n}$ Wiley and Sons.

62. Babich, M.W.; Hwang, S.W.; Mounts, R.D. (1992) The thermal analysis of energy storage materials by differential scanning calorimetry. Thermochim. Acta. 210, 77-82. https://doi.org/10.1016/0040-6031(92)80278-5.

63. Sarı, A.; Alkan, C.; Altıntaş, A. (2014) Preparation, characterization and latent heat thermal energy storage properties of micro-nanoencapsulated fatty acids by polystyrene shell. Appl. Therm. Eng. 73, 1160-1168. https://doi.org/10.1016/j. applthermaleng.2014.09.005

64. Sari, A.; Karaipekli, A. (2008) Preparation and thermal properties of capric acid/palmitic acid eutectic mixture as a phase change energy storage material. Mater. Lett. 62, 903-906. https://doi.org/10.1016/j.matlet.2007.07.025.

65. Pielichowski, K.; Flejtuch, K. (2003) Differential Scanning Calorimetry Study of Blends of Poly (ethylene glycol) with Selected Fatty Acids. Macromol. Mater. Eng. 288, 259-264. 
66. Sari, A.; Kaygusuz, K. (2003) Some fatty acids used for latent heat storage: Thermal stability and corrosion of metals with respect to thermal cycling. Renew. Energy. 28, 939-948. https://doi.org/10.1016/S0960-1481(02)00110-6.

67. Keleş, S.; Kaygusuz, K.; Sari, A. (2005) Lauric and myristic acids eutectic mixture as phase change material for lowtemperature heating applications. Int. J. Energy Res. 29, 857-870. https://doi.org/10.1002/er.1111.

68. Sari, A.; Kaygusuz, K. (2001) Thermal performance of myristic acid as a phase change material for energy storage application. Renew. Energy. 24, 303-317. https://doi. org/10.1016/S0960-1481(00)00167-1.

69. Sari, A.; Kaygusuz, K. (2002) Thermal performance of palmitic acid as a phase change energy storage material. Energy Convers. Manag. 43, 863-876. https://doi.org/10.1016/ S0196-8904(01)00071-1.

70. Cao, L.; Tang, F.; Fang, G. (2014) Preparation and characteristics of microencapsulated palmitic acid with $\mathrm{TiO} 2$ shell as shape-stabilized thermal energy storage materials. Sol. Energy Mater. Sol. Cells. 123, 183-188. https://doi. org/10.1016/j.solmat.2014.01.023.

71. Sari, A.; Kaygusuz, K. (2001) Thermal energy storage system using stearic acid as a phase change material. Sol. Energy. 71, 365-376. https://doi.org/10.1016/S0038-092X(01)00075-5.

72. Babich, M.W.; Hwang, S.W.; Mounts, R.D. (1992) The search for novel energy storage materials differential scanning caldrimetry. 210, 83-88.

73. Feldman, D.; Shapiro, M.M.; Banu, D. (1986) Organic phase change materials for thermal energy storage. Sol. Energy Mater. 13, 1-10. https://doi.org/10.1016/0165-1633 (86)90023-7.

74. Kenisarin, M.M. (1993) Short-Term Storage of Solar Energy. 1. Low Temperature Phase-Change Materials. Geliotekhnika. 29, 46-64.

75. Khudhair, A.M.; Farid, M.M. (2004) A review on energy conservation in building applications with thermal storage by latent heat using phase change materials. Energy Convers. Manag. 45, 263-275. https://doi.org/10.1016/ S0196-8904(03)00131-6.

76. Hawes, D.W. Feldman, D; Banu, D. (1993) Latent heat storage in building materials. Energy Build. 20, 77-86. https://doi.org/10.1016/0378-7788(93)90040-2.

77. Babich, M.W.; Benrashid, R.; Mounts, R.D. (1994) DSC studies of new energy storage materials. Part 3. Thermal and flammability studies. Thermochim. Acta. 243, 193-200. https://doi.org/10.1016/0040-6031(94)85054-2.

78. Alkan, C.; Kaya, K.; Sari, A. (2008) Preparation and thermal properties of ethylene glycole distearate as a novel phase change material for energy storage. Mater. Lett. 62, 1122-1125. https://doi.org/10.1016/j.matlet.2007.07.061.

79. Salaün, F.; Bedek, G.; Devaux, E.; Dupont, D.; Gengembre, L. (2011) Microencapsulation of a cooling agent by interfacial polymerization: Influence of the parameters of encapsulation on poly(urethane-urea) microparticles characteristics. J. Memb. Sci. 370, 23-33. https://doi.org/ 10.1016/j.memsci.2010.11.033

80. Kaizawa, A.; Maruoka, N.; Kawai, A ; Kamano, H ; Jozuka, T.; Senda, T.; Akiyama, T. (2007) Thermophysical and heat transfer properties of phase change material candidate for waste heat transportation system. Heat Mass Transf. 44, 763-769. https://doi.org/10.1007/s00231-007-0311-2.

81. Kakiuchi, H.; Yamayaki, M.; Yabe, M.; Chihara, S.; Terunuma, Y.; Sakata, Y.; Usami, T. A study of erythritol as phase change material. in: 2nd Work. IEA ECES IA Annex 10, Sofia (Bulgaria)

82. Pielichowski, K.; Flejtuch, K. (2002) Differential scanning calorimetry studies on poly(ethylene glycol) with different molecular weights for thermal energy storage materials. Polym. Adv. Technol. 13, 690-696. https://doi.org/10.1002/ pat.276.

83. Zeng, J.L.; Zhang, J.; Liu, Y.Y.; Cao, Z.X.; Zhang, Z.H.; Xu, F.; Sun, L.X. (2008) Polyaniline/1-tetradecanol composites: Form-stable PCMS and electrical conductive materials. $J$. Therm. Anal. Calorim. 91, 455-461. https://doi.org/10.1007/ s10973-007-8495-8.
84. Farid, M.; Hamad, F.; Abu-Arabi, M. (1998) Phase change cool storage using dimethyl-sulfoxide. Energy Convers. Manag. 39, 819-826. http://www.sciencedirect. com/science?_ob=ArticleURL\&_udi=B6V2P-3SYXFDSF\&_user $=984461 \&$ \&coverDate $=06 \% 2 \mathrm{~F} 10 \% 2 \mathrm{~F} 1998 \&$ _ rdoc $=13 \&$ fmt $=$ high $\&$ orig $=$ browse $\&$ origin $=$ browse $\&$ zone $=$ rslt_list_item \&_srch $=$ doc-info $(\% \overline{2} 3$ toc $\% 235708 \% 2 \overline{3}$ $1998 \% 23999609991 \% 2314010 \% 23$ FLA $\% 23$ display $\% 23 \mathrm{~V}$.

85. Kenar, J.A. (2010) Latent heat characteristics of biobased oleochemical carbonates as potential phase change materials. Sol. Energy Mater. Sol. Cells. 94, 1697-1703. https:// doi.org/10.1016/j.solmat.2010.05.031.

86. Belton, G.R.; Ajami, F. (1973) Thermochemistry of Salt Hydrates (Report no. NSF/RANN/SE/GI27976/TR/73/4).

87. Tyagi, V. V.; Buddhi, D. (2008) Thermal cycle testing of calcium chloride hexahydrate as a possible PCM for latent heat storage. Sol. Energy Mater. Sol. Cells. 92, 891-899. https://doi.org/10.1016/j.solmat.2008.02.021.

88. Halawa, E.; Saman, W. (2011) Thermal performance analysis of a phase change thermal storage unit for space heating. Renew. Energy. 36, 259-264. https://doi.org/10.1016/j. renene.2010.06.029.

89. Bilen, K.; Takgil, F.; Kaygusuz, K. (2008) Thermal Energy Storage Behavior of $\mathrm{CaCl} 2.6 \mathrm{H} 2 \mathrm{O}$ during Melting and Solidification. Energy Sources Part A. 775-787. https://doi. org/10.1080/15567030601082175.

90. Farid, M.M.; Khudhair, A.M.; Razack, S.A.K.; Al-Hallaj, S. (2004) A review on phase change energy storage: Materials and applications. Energy Convers. Manag. 45, 1597-1615. https://doi.org/10.1016/j.enconman.2003.09.015

91. Heckenkamp, J.; Baumann, H. (1997) Latentwärmespeicher. Sonderdruck aus Nachrichten. 11, 1075-1081.

92. Etheridge, D; Murphy, K. Reay, D (2006) A PCM/heat pipe cooling system for reducing air conditioning in buildings: review of options and report on field tests. Build. Serv. Eng. Res. Technol. 27, 27-39. https://doi.org/10.1191/ 0143624406bt142oa.

93. Qu, S.; Ma, F.; Ji, R.; Wang, D.; Yang, L. (2015) System design and energy performance of a solar heat pump heating system with dual-tank latent heat storage. Energy Build. 105, 294-301. https://doi.org/10.1016/j.enbuild.2015. 07.040 .

94. Guion, J.; Sauzade, J.D.; Laügt, M. (1983) Critical examination and experimental determination of melting enthalpies and entropies of salt hydrates. Thermochim. Acta. 67, 167-179. https://doi.org/10.1016/0040-6031(83)80096-3.

95. Yinping, Z.; Yi, J. (1999) A simple method the T -history method of determining the heat of fusion specific heat and thermal conductivity of phase-change materials. Meas. Sci. Technol. 10, 201-205. https://doi.org/10.1088/ 0957-0233/10/3/015.

96. Cabeza, L.F.; Svensson, G.; Hiebler, S.; Mehling, H. (2003) Thermal performance of sodium acetate trihydrate thickened with different materials as phase change energy storage material. Appl. Therm. Eng. 23, 1697-1704. https://doi. org/10.1016/S1359-4311(03)00107-8.

97. Dannemand, M.; Fan, J.; Furbo, S.; Reddi, J. (2014) Validation of a CFD model simulating charge and discharge of a small heat storage test module based on a sodium acetate water mixture. Energy Procedia. 57, 2451-2460. https:// doi.org/10.1016/j.egypro.2014.10.254.

98. Davidson, J.H.; Quinnell, J.; Burch, J.; Zondag, H. a; Boer, R. De; Finck, C.; Cuypers, R.; Cabeza, L.F.; Heinz, A.; Jahnig, D.; Furbo, S.; Bertsch, F. Development of Space Heating and Domestic Hot Water Systems with Compact Thermal Energy Storage. in: Compact Therm. Energy Storage Mater. Dev. Syst. Integr., Report of the IEA SHC/ ECES programme - Task 42/Annex 24. http://www.iea-shc. org/task42 [accessed April 2015].

99. Gutierrez, A.; Miró, L.; Gil, A.; Rodríguez-Aseguinolaza, J.; Barreneche, C.; Calvet, N.; Py, X.; Inés Fernández, A. Grágeda, M.; Ushak, S.; Cabeza, L.F. (2016) Advances in the valorization of waste and by-product materials as thermal energy storage (TES) materials. Renew. Sustain. Energy Rev. 59, 763-783. https://doi.org/10.1016/j.rser.2015.12.071. 
100. (n.d.) GoodFellow. Material information. http://www.goodfellow.com/E/Gallium.html (accessed October 6, 2016)

101. (n.d.) Bolton Metal Product CO. http://www.boltonmetalproducts.com/Specifications.html (accessed October 6, 2016).

102. (n.d.) Cerro Alloys. http://www.csalloys.com/specifications. html (accessed October 6, 2016)

103. Roxas-Dimaano, M.N.; Watanabe, T. (2002) The capric and lauric acid mixture with chemical additives as latent heat storage materials for cooling application. Energy. 27, 869-888. https://doi.org/10.1016/S0360-5442(02)00024-5.

104. Sari, A. (2005) Eutectic mixtures of some fatty acids for low temperature solar heating applications: Thermal properties and thermal reliability. Appl. Therm. Eng. 25, 2100-2107. https://doi.org/10.1016/j.applthermaleng.2005.01.010.

105. Baran, G.; Sari, A. (2003) Phase change and heat transfer characteristics of a eutectic mixture of palmitic and stearic acids as PCM in a latent heat storage system. Energy Convers. Manag. 44, 3227-3246. https://doi.org/10.1016/ S0196-8904(03)00104-3.

106. Sari, A. (2003) Thermal characteristics of a eutectic mixture of myristic and palmitic acids as phase change material for heating applications. Appl. Therm. Eng. 23, 1005-1017. https://doi.org/10.1016/S1359-4311(03)00031-0.

107. Sari, A.; Kaygusuz, K. (2002) Thermal performance of a eutectic mixture of lauric and stearic acids as PCM encapsulated in the annulus of two concentric pipes. Sol. Energy. 72, 493-504. https://doi.org/10.1016/S0038-092X(02)00026-9.

108. Sarı, A.; Alkan, C.; Ozcan, A.N. (2015) Synthesis and characterization of micro/nano capsules of PMMA/capricstearic acid eutectic mixture for low temperature-thermal energy storage in buildings. Energy Build. 90, 106-113. https://doi.org/10.1016/j.enbuild.2015.01.013.

109. Sari, A.; Kaygusuz, K. (2006) Thermal energy storage characteristics of myristic and stearic acids eutectic mixture for low temperature heating applications. Chinese J. Chem. Eng. 14, 270-275. https://doi.org/10.1016/S1004-9541(06)60070-0.

110. Kaygusuz, K.; Sari, A. (2006) Thermal Energy Storage Performance of Fatty Acids as a Phase Change Material. Energy Sources, Part A Recover. Util. Environ. Eff. 28, 105-116. https://doi.org/10.1080/009083190913971.

111. Li, J.H.; Zhang, G. en; Wang, J.Y. (1991) Investigation of a eutectic mixture of sodium acetate trihydrate and urea as latent heat storage. Sol. Energy. 47, 443-445. https://doi. org/10.1016/0038-092X(91)90112-A.

112. Bales, C.; Gantenbein, P.; Jaenig, D.; Kerskes, H.; Summer, K.; Van Essen, M. Weber R. (2008) Laboratory Tests of Chemical Reactions and Prototype Sorption Storage Units. A Rep. IEA Sol. Heat. Cool. Program. - Task 32 Adv. Storage Concepts Sol. Low Energy Build. http://drxvzvm. iea-shc.org/data/sites/1/publications/task32-b4.pdf

113. Bales, C.; Gantenbein, P.; Jaehnig, D.; Kerskes, H.; van Essen, M.; Weber, R.; Zondag, H. Chemical and Sorption Storage. in: EUROSUN 2008. 1st Int. Congr. Heating, Cool. Build., Lisbon, Portugal.

114. Grande, C. a. (2012) Advances in Pressure Swing Adsorption for Gas Separation. ISRN Chem. Eng. 2012, 1-13. https:// doi.org/10.5402/2012/982934.

115. Abedin, A.H.; Rosen, M.A. (2012) Closed and open thermochemical energy storage: Energy- and exergy-based comparisons. Energy. 41, 83-92. https://doi.org/10.1016/j. energy.2011.06.034.

116. Semprini, S.; Lehmann, C.; Beckert, S.; Kolditz, O.; Gläser, R. Kerskes, H ; Nagel, T. (2017) Numerical modelling of water sorption isotherms of zeolite $13 \mathrm{XBF}$ based on sparse experimental data sets for heat storage applications. Energy Convers. Manag. 150, 392-402. https://doi.org/10.1016/j. enconman.2017.08.033

117. Frazzica, A.; Freni, A. (2017) Adsorbent working pairs for solar thermal energy storage in buildings. Renew. Energy. 110, 87-94. https://doi.org/10.1016/j.renene.2016.09.047.

118. Zettl, B.; Englmair, G.; Steinmaurer, G. (2014) Development of a revolving drum reactor for open-sorption heat storage processes. Appl. Therm. Eng. 70, 42-49. https://doi. org/10.1016/j.applthermaleng.2014.04.069.
119. Wagner, W.; Janhig, W.; Isaksson, D.; Hausner, R. (2006) Modularer energiespeicher nach dem sorptionprinzip mit hoher energiedichte - Modestore, Technology report.

120. Lim, K.; Che, J.; Lee, J. (2017) Experimental study on adsorption characteristics of a water and silica-gel based thermal energy storage (TES) system. Appl. Therm. Eng. 110, 80-88. https://doi.org/10.1016/j.applthermaleng.2016. 08.098 .

121. Sapienza, A.; Velte, A.; Girnik, I.; Frazzica, A.; Füldner, G.; Schnabel, L.; Aristov, Y. (2017) "Water - Silica Siogel" working pair for adsorption chillers: Adsorption equilibrium and dynamics. Renew. Energy. 110, 40-46. https://doi. org/10.1016/j.renene.2016.09.065.

122. Deshmukh, H.; Maiya, M.P.; Srinivasa Murthy, S. (2017) Study of sorption based energy storage system with silica gel for heating application. Appl. Therm. Eng. 111, 1640-1646. https://doi.org/10.1016/j.applthermaleng.2016.07.069.

123. Fernandes, M.S.; Brites, G.J.V.N.; Costa, J.J.; Gaspar, A.R.; Costa, V.A.F. (2016) A thermal energy storage system provided with an adsorption module - Dynamic modeling and viability study. Energy Convers. Manag. 126, 548-560. https://doi.org/10.1016/j.enconman.2016.08.032.

124. Hauer, A. Adsorption Systems for Tes-Design and Demonstration Projects. in: Therm. Energy Storage Sustain. Energy Consum., Springer, Netherlands.: 409-427. https:// doi.org/10.1007/978-1-4020-5290-3 25.

125. Köll, R.; van Helden, W.; Engel, G.; Wagner, W.; Dang, B.; Jänchen, J.; Kerskes, H.; Badenhop, T.; Herzog, T. (2017) An experimental investigation of a realistic-scale seasonal solar adsorption storage system for buildings. Sol. Energy. 155, 388-397. https://doi.org/10.1016/j.solener. 2017.06.043.

126. Tatsidjodoung, P. Le Pierrès, N.; Heintz, J; Lagre, D.; Luo, L.; Durier, F. (2016) Experimental and numerical investigations of a zeolite 13X/water reactor for solar heat storage in buildings. Energy Convers. Manag. 108, 488-500. https:// doi.org/10.1016/j.enconman.2015.11.011.

127. Gaeini, M.; Zondag, H.A.; Rindt, C.C.M. (2016) Effect of kinetics on the thermal performance of a sorption heat storage reactor. Appl. Therm. Eng. 102, 520-531. https:// doi.org/10.1016/j.applthermaleng.2016.03.055.

128. Finck, C.; Henquet, E.; Van Soest, C.; Oversloot, H.; De Jong, A.J.; Cuypers, R.; Van T'Spijker, H. (2014) Experimental results of a $3 \mathrm{kWh}$ thermochemical heat storage module for space heating application. Energy Procedia. 48, 320-326. https://doi.org/10.1016/j.egypro.2014.02.037.

129. Vanhoudt, D.; Claessens, B.; De Ridder, F.; Reynders, G.; Cuypers, R.; Oversloot, H.; Finck, C.; van Soest, C.; de Jong, A.; Henquet, E.; van 't Spijker, H.; Koene, F.; Smeding, S.; Zondag, H. (2014) Energy-Hub for residential and commercial districts and transport. D3.2 Report on a combination of thermal storage techniques and components.

130. (n.d.) Energy-Hub for residential and commercial districts and transport - E-hub project (2010-2014) - Collaborative European project (7FP). http://www.e-hub.org/index.html (accessed June 9, 2016)

131. Helden, W. Van; Wagner, W.; Schubert, V.; Krampe-Zadler, C.; Kerskes, H.; Bertsch, F.; Mette, B.; Jänchen, J. (2014) Experimental tests on a solid sorption prototype for seasonal solar thermal storage. Eurotherm Semin. \#99 Adv. Therm. Energy Storage. 1-8.

132. (n.d.) Compact thermal storage technologies - COMTES Project (2012-2016) - Collaborative European project (7FP). http://comtes-storage.eu/comtes-project/ (accessed June 9, 2016).

133. Johannes, K.; Kuznik, F.; Hubert, J.-L.; Durier, F.; Obrecht, C. (2015) Design and characterisation of a high powered energy dense zeolite thermal energy storage system for buildings. Appl. Energy. 159, 80-86. https://doi.org/10.1016/j. apenergy.2015.08.109.

134. N'Tsoukpoe, K.E.; Le Pierrès, N.; Luo, L. (2013) Experimentation of a $\mathrm{LiBr}-\mathrm{H} 2 \mathrm{O}$ absorption process for long-term solar thermal storage: Prototype design and first results. Energy. 53, 179-198. https://doi.org/10.1016/j. energy.2013.02.023 
135. Weber, R.; Dorer, V. (2008) Long-term heat storage with $\mathrm{NaOH}$. Vacuum. 82, 708-716. https://doi.org/10.1016/j. vacuum.2007.10.018.

136. Daguenet-Frick, X.; Gantenbein, P.; Frank, E.; Fumey, B.; Weber, R.; Williamson, T. Seasonal Thermal Energy Storage with Aqueous Sodium Hydroxide. Reaction Zone development, manufacturing and first experimental assessments. in: EuroSun 2014. ISES Conf. Proc., Aix-les-Bain, France.

137. Daguenet-Frick, X.; Gantenbein, P.; Frank, E.; Fumey, B.; Weber, R.; Williamson, T. (2014) Reaction zone development for an aqueous sodium hydroxide seasonal thermal energy storage. Energy Procedia. 57, 2426-2435. https://doi. org/10.1016/j.egypro.2014.10.251.

138. Fumey, B.; Weber, R.; Gantenbein, P.; Daguenet-Frick, X.; Williamson, T.; Dorer, V. (2014) Closed sorption heat storage based on aqueous sodium hydroxide. Energy Procedia. 48, 337-346. https://doi.org/10.1016/j.egypro. 2014.02.039.

139. Berg Johansen, J.; Furbo, S. (2015) COMTES - Deliverable 5.1 : Description of experimental systems. http://comtesstorage.eu/wordpress/wp-content/uploads/2015/06/D5-1final-Description-of-experimental-systems.pdf.

140. Fumey, B.; Weber, R.; Baldini, L. (2017) Liquid sorption heat storage - A proof of concept based on lab measurements with a novel spiral fined heat and mass exchanger design. Appl. Energy. 200, 215-225. https://doi.org/10.1016/j. apenergy.2017.05.056.

141. Quinnell, J.A.; Davidson, J.H. (2012) Mass transfer during sensible charging of a hybrid absorption/sensible storage tank. Energy Procedia. 30, 353-361. https://doi.org/10.1016/j. egypro.2012.11.042

142. Quinnell, J. a.; Davidson, J.H.; Burch, J. (2011) Liquid Calcium Chloride Solar Storage: Concept and Analysis. J. Sol. Energy Eng. 133, 11010. https://doi.org/10.1115/1.4003292.

143. Richter, M.; Bouché, M.; Linder, M. (2016) Heat transformation based on $\mathrm{CaCl} 2 / \mathrm{H} 2 \mathrm{O}$ - Part A: Closed operation principle. 102, 615-621. https://doi.org/10.1016/j. applthermaleng.2016.03.076.

144. N'Tsoukpoe, K.E.; Le Pierrès, N.; Luo, L. (2012) Numerical dynamic simulation and analysis of a lithium bromide/ water long-term solar heat storage system. Energy. 37, 346358. https://doi.org/10.1016/j.energy.2011.11.020.

145. Xu, S.M.; Huang, X.D.; Du, R. (2011) An investigation of the solar powered absorption refrigeration system with advanced energy storage technology. 85, 1794-1804. https:// doi.org/10.1016/j.solener.2011.04.022.

146. Perier-Muzet, M.; Le Pierres, N. (2016) Modeling and analysis of energetic and exergetic efficiencies of a $\mathrm{LiBr} / \mathrm{H} 20$ absorption heat storage system for solar space heating in buildings. Energy Effic. 9, 281-299. https://doi.org/10.1007/ s12053-015-9362-2.

147. Jonsson, S.; Kaarebring-Olsson, M.; Olsson, R. 2000, A chemical heat pump.

148. Bales, C.; Ayadi, O. (2009) Modelling of a Commercial Absorption Heat Pump with Integral Storage. Effstock 2009 - 11th Int. Conf. Energy Storage. Stockholm.

149. Hui, L.; Edem, N.K.; Nolwenn, L.P.; Luo, L. (2011) Evaluation of a seasonal storage system of solar energy for house heating using different absorption couples. Energy Convers. Manag. 52, 2427-2436. https://doi.org/10.1016/j. enconman.2010.12.049

150. Scapino, L.; Zondag, H.A.; Bael, J. Van; Diriken, J.; Rindt, C.C.M. (2017) Energy density and storage capacity cost comparison of conceptual solid and liquid sorption seasonal heat storage systems for low-temperature space heating. 76, 1314-1331. https://doi.org/10.1016/j.rser.2017.03.101.

151. Esaki, T.; Yasuda, M.; Kobayashi, N. (2017) Experimental evaluation of the heat output/input and coefficient of performance characteristics of a chemical heat pump in the heat upgrading cycle of $\mathrm{CaCl} 2$ hydration. Energy Convers. Manag. 150, 365-374. https://doi.org/10.1016/j. enconman.2017.08.013.

152. Bouché, M.; Richter, M.; Linder, M. (2016) Heat transformation based on $\mathrm{CaCl} 2 / \mathrm{H} 2 \mathrm{O}$ - Part B: Open operation principle. Appl. Therm. Eng. 102, 641-647. https://doi.org/ 10.1016/j.applthermaleng.2016.03.102.

153. Mette, B.; Kerskes, H.; Drück, H.; Müller-Steinhagen, H. (2013) New highly efficient regeneration process for thermochemical energy storage. Appl. Energy. 109, 352-359. https://doi.org/10.1016/j.apenergy.2013.01.087.

154. Cot-Gores, J.; Castell, A.; Cabeza, L.F. (2012) Thermochemical energy storage and conversion: A-stateof-the-art review of the experimental research under practical conditions. Renew. Sustain. Energy Rev. 16, 5207-5224. https://doi.org/10.1016/j.rser.2012.04.007.

155. van Essen, V.M.; Zondag, H.A.; Gores, J.C.; Bleijendaal, L.P.J.; Bakker, M.; Schuitema, R.; van Helden, W.G.J.; He, Z.; Rindt, C.C.M. (2009) Characterization of MgSO4 Hydrate for Thermochemical Seasonal Heat Storage. J. Sol. Energy Eng. 131, 1-7. https://doi.org/10.1115/1.4000275.

156. Zondag, H.; Kikkert, B.; Smeding, S.; Boer, R. de; Bakker, M. (2013) Prototype thermochemical heat storage with open reactor system. Appl. Energy. 109, 360-365. https:// doi.org/10.1016/j.apenergy.2013.01.082

157. Barreneche, C.; Fernández, A.I.; Cabeza, L.F.; Cuypers, R. (2015) Thermophysical characterization and thermal cycling stability of two TCM: CaCl2 and zeolite. Appl. Energy. 137, 726-730. https://doi.org/10.1016/j.apenergy.2014.09.025.

158. Ruiz-Agudo, E.; Martin-Ramos, J.D.; Rodriguez-Navarro, C. (2007) Mechanism and kinetics of dehydration of epsomite crystals formed in the presence of organic additives. J. Phys. Chem. B. 111, 41-52. https://doi.org/10.1021/jp064460b.

159. van Essen, V.M.M.; Zondag, H. a. a.; Schuitema, R.; van Helden, W.G.J.G.J.; Rindt, C.C.M.C.M.; Essen, V.M. Van; Zondag, H. a. a.; Schuitema, R.; Helden, W.G.J. Van; Rindt, C.C.M.C.M. (2008) Materials for thermochemical storage: characterization of magnesium sulfate. Proc. Eurosun. 4-9.

160. Okhrimenko, L.; Favergeon, L.; Johannes, K.; Kuznik, F.; Pijolat, M. (2017) Thermodynamic study of MgSO4 $\cdot \mathrm{H} 2 \mathrm{O}$ system dehydration at low pressure in view of heat storage. Thermochim. Acta. 656, 135-143. https://doi.org/10.1016/j. tca.2017.08.015.

161. Ferchaud, C.J.; Scherpenborg, R.A.A.; Zondag, H.A.; De Boer, R. (2014) Thermochemical seasonal solar heat storage in salt hydrates for residential applications - Influence of the water vapor pressure on the desorption kinetics of MgSO4.7H2O. Energy Procedia. 57, 2436-2440. https:// doi.org/10.1016/j.egypro.2014.10.252.

162. Kerskes, H.; Mette, B.; Bertsch, F.; Asenbeck, S.; Drück, H. (2012) Chemical energy storage using reversible solid/ gas-reactions (CWS) - Results of the research project. Energy Procedia. 30, 294-304. https://doi.org/10.1016/j. egypro.2012.11.035.

163. Willers, E.; Wanner, M.; Groll, M. (1999) Multi-hydride thermal wave device for simultaneous heating and cooling. J. Alloys Compd. 293, 915-918. https://doi.org/10.1016/ S0925-8388(99)00440-5.

164. Lammak, K.; Wongsuwan, W.; Kiatsiriroj, T. Investigation of modular chemical energy storage performance. in: Proc. Jt. Int. Conf. Energy Environ., Hua Hin, Thailand;

165. Zhang, P.; Wang, C.; Wang, R. (2007) Composite reactive block for heat transformer system and improvement of system performance. J. Chem. Eng. Japan. 40, 1275-1280. https://doi.org/10.1252/jcej.07WE148.

166. Mauran, S.; Lahmidi, H.; Goetz, V. (2008) Solar heating and cooling by a thermochemical process. First experiments of a prototype storing $60 \mathrm{kWh}$ by a solid/gas reaction. Sol. Energy. 82, 623-636. https://doi.org/10.1016/j.solener.2008.01.002.

167. Wang, L.W.; Wang, R.Z.; Wu, J.Y.; Wang, K. (2004) Compound absorbent for adsorption ice maker on fishing boats. Int. J. Refrig. 27, 401-408. https://doi.org/10.1016/j. ijrefrig.2003.11.010.

168. de Boer, R.; Haije, W.G.; Veldhuis, J.B.J.; Smeding, S.F. Solid-sorption cooling with integrated thermal storage: the SWEAT prototype. in: Proc. HPC 2004-3rd Int. Heat Powered Cycles Conf., Larnaca, Cyprus.

169. Wu, H.; Wang, S.; Zhu, D. (2007) Effects of impregnating variables on dynamic sorption characteristics and storage properties of composite sorbent for solar heat storage. 
Sol. Energy. 81, 864-871. https://doi.org/10.1016/j.solener. 2006.11.013

170. Courbon, E.; D’Ans, P.; Permyakova, A.; Skrylnyk, O.; Steunou, N.; Degrez, M.; Frère, M. (2017) Further improvement of the synthesis of silica gel and $\mathrm{CaCl} 2$ composites: Enhancement of energy storage density and stability over cycles for solar heat storage coupled with space heating applications. Sol. Energy. 157, 532-541. https://doi.org/10.1016/j. solener.2017.08.034.

171. Jabbari-Hichri, A.; Bennici, S.; Auroux, A. (2017) CaCl 2 -containing composites as thermochemical heat storage materials. Sol. Energy Mater. Sol. Cells. 172, 177-185. https://doi.org/10.1016/j.solmat.2017.07.037.

172. Lizana, J.; Chacartegui, R.; Barrios-Padura, A.; Valverde, J.M. (2017) Advances in thermal energy storage materials and their applications towards zero energy buildings: A critical review. Appl. Energy. 203, 219-239. https://doi. org/10.1016/j.apenergy.2017.06.008.

173. Valverde, J.M.; Castellanos, A. (2007) Random loose packing of cohesive granular materials. Europhys. Lett. 75, 985-991. https://doi.org/10.1209/ep1/i200610208-4. 